\title{
A Survey and Comparison of Methods for Quantitative Texture Analysis
}

A Thesis

Presented to

The Faculty of the School of Engineering and Applied Science

University of Virginia

In Partial Fulfillment

Of the Requirements for the Degree

Master of Science in Materials Science and Engineering

By

John Rogers Einhorn

July 2017 


\section{Abstract}

Numerous methods for measuring and analyzing texture are explored and discussed. Traditional pole figure inversion methods are compared against more recent developments in quantitative texture analysis involving the Rietveld whole pattern refinement method. A modified equal area pole figure collection strategy is compared to the conventional equiangular method using laboratory X-ray diffraction (XRD). The two measurement strategies were found to yield qualitatively similar orientation distribution functions (ODF), and the former set takes about half the time to collect. However, the estimated texture was found to be strongly dependent on the ODF computation parameters used in MTEX. The texture evolution during compression of a high specific strength Mgbase alloy containing long period stacking order (LPSO) phase is studied using synchrotron XRD. The initial texture was found to be much sharper than previously published results, demonstrating the importance of using a "direct method" to compute the OD for this material. The evolution of the texture of the Mg and LPSO phases suggests that the deformation is accommodated almost entirely by twinning in the former case and by dislocation slip, elastic deformation, and possibly some twinning in the latter. The texture of $\alpha$-uranium foils is measured via X-ray (both $\mathrm{Cu}$ - and $\mathrm{Mo}-\mathrm{K}_{\alpha}$ radiation) and neutron diffraction, and the results are analyzed with MTEX and MAUD (Rietveld), respectively. The three radiation sources are found to yield slightly different results, based on probing different depths of the foils. Finally, the capability of measuring texture on the Neutron Residual Stress Mapping Facility (NRSF2) and Nanoscale Ordered Materials Diffractometer (NOMAD) instruments at the Oak Ridge National Laboratory (ORNL) is demonstrated by comparing measurements to those obtained via conventional XRD methods. Pole figure inversion in MTEX (for XRD and NRSF2) and Rietveld analysis in MAUD (for NOMAD) are employed for analysis, and the results are quantitatively compared. Reasonable agreement is found across all methods, and the differences between methods are attributed mostly to differences in the analysis procedure. The implications of slight variations in texture, as estimated by the various techniques, are explored through calculations of tensor properties 
and comparisons with predicted deformation texture evolutions. Finally, suggestions for improving the data collection speed by collecting less data are explored for both instruments. 


\section{Table of Contents}

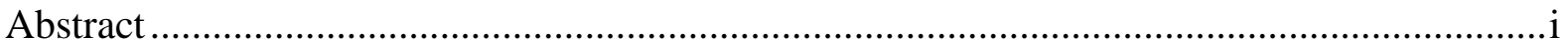

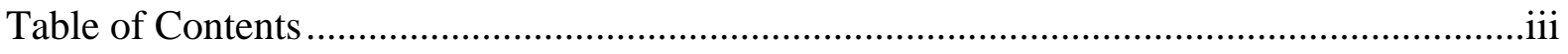

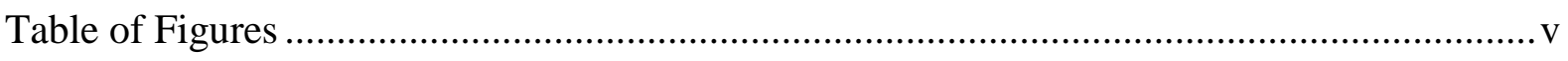

List of Tables ............................................................................................................ii

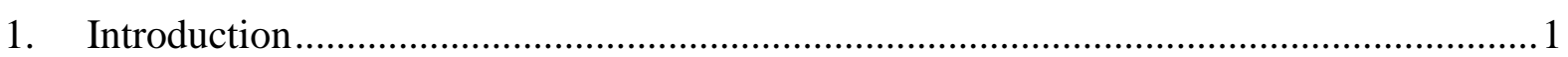

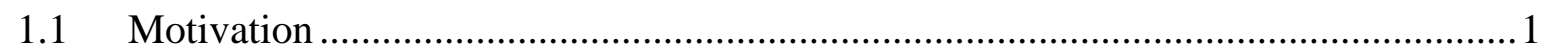

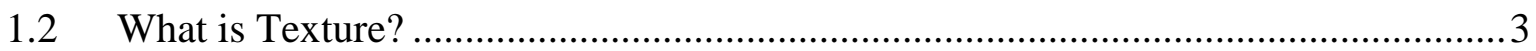

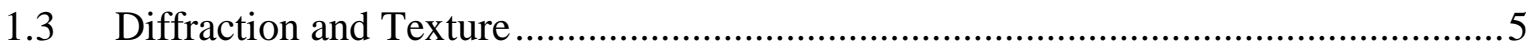

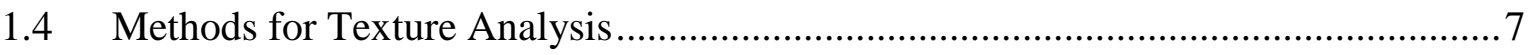

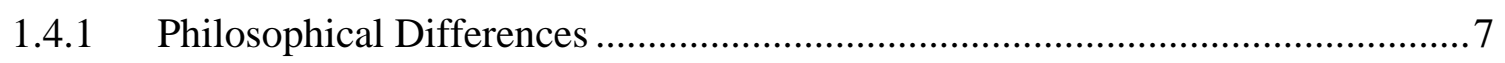

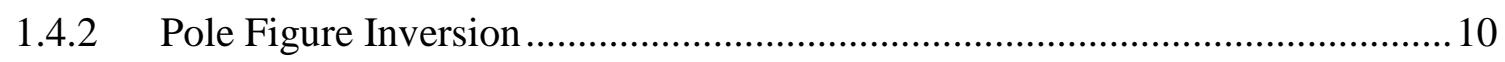

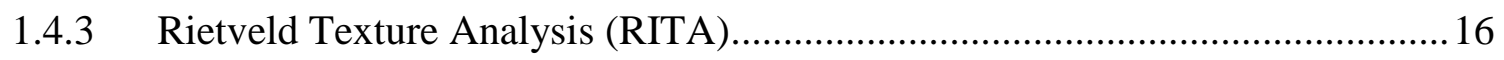

1.4.4 Analysis of Individual Grains .................................................................... 19

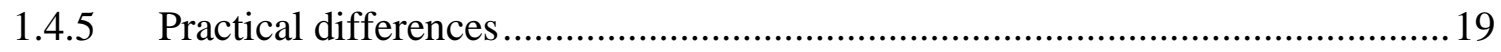

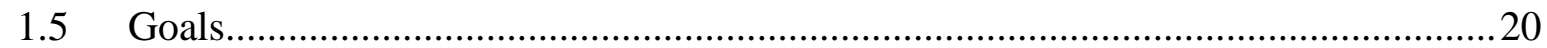

2. Pole Figure Inversion using MTEX: Comparison of Various Measurement and Analysis Procedures.

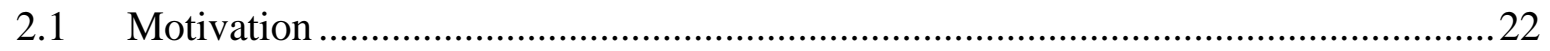

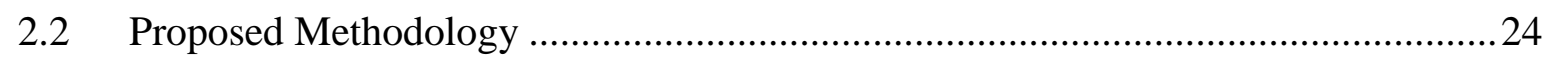

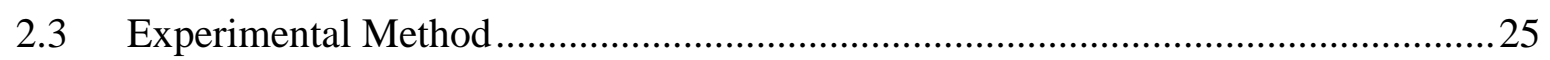

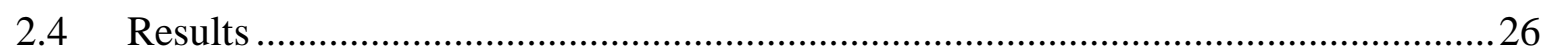

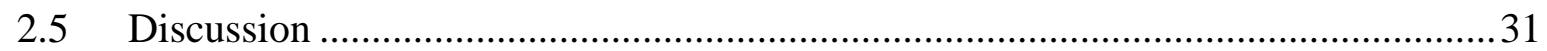

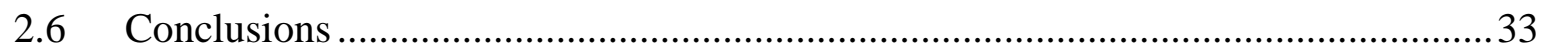

3. In-situ Texture Analysis of Long-Period Stacking Order Mg Alloys via Synchrotron X-

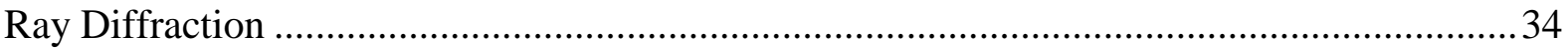

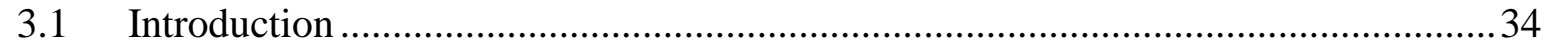

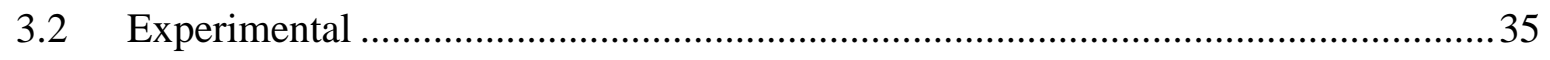

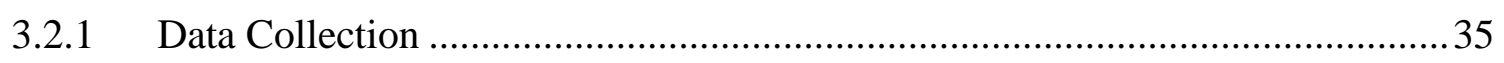

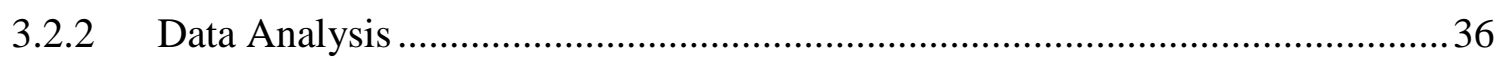

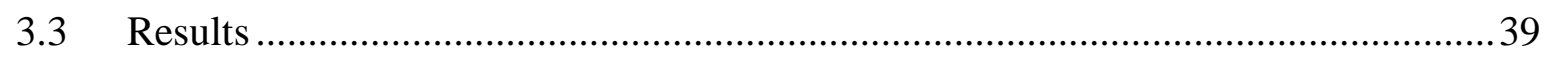

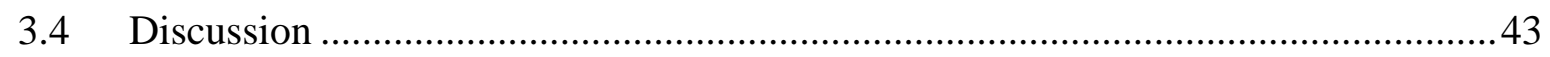

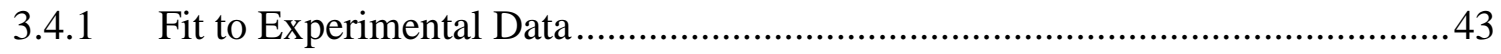

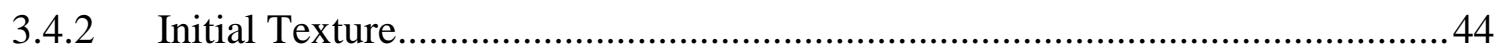

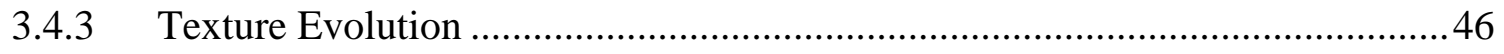

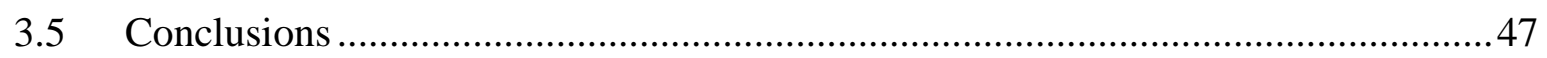


4. Crystallographic Texture of Straight-Rolled $\alpha$-Uranium Foils via Neutron and X-ray

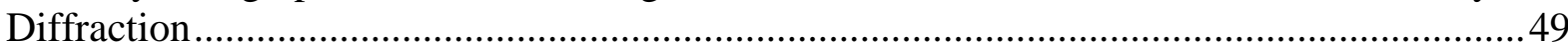

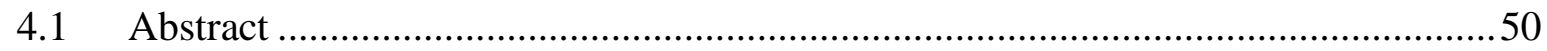

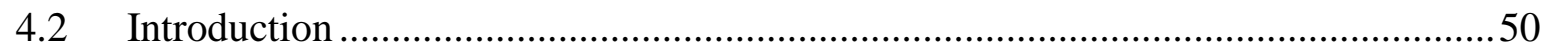

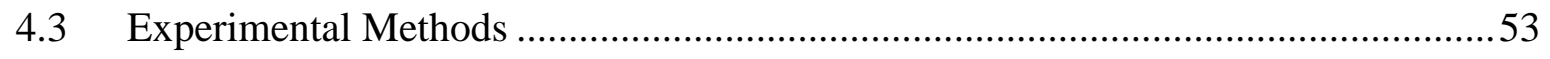

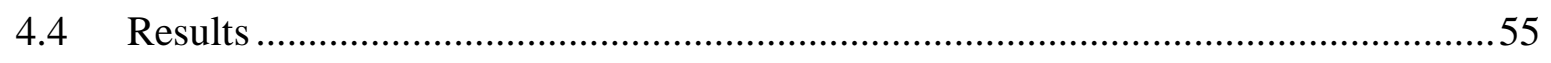

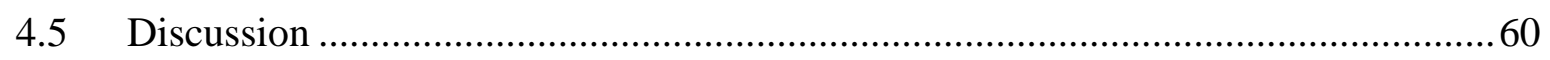

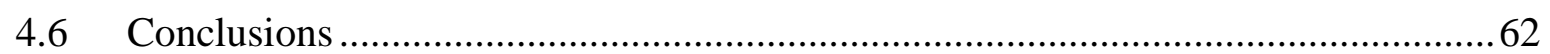

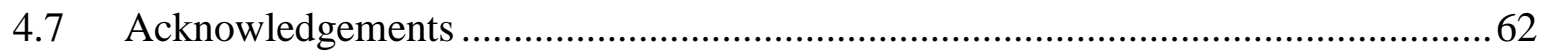

5. Crystallographic Texture Measurement via Neutron Diffraction at the Oak Ridge

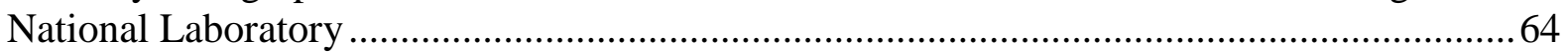

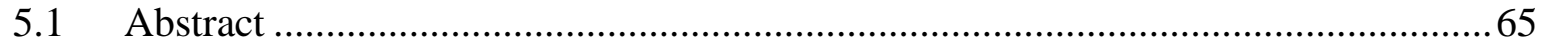

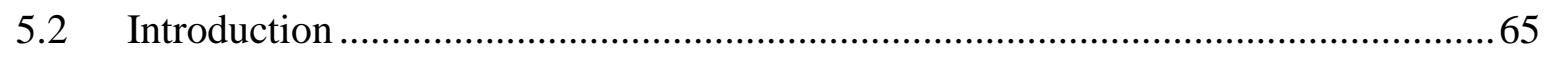

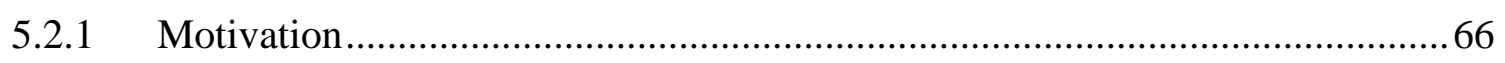

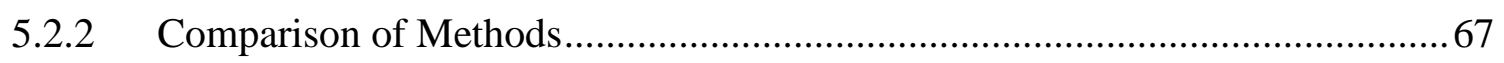

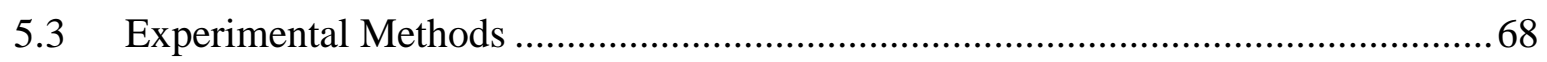

5.3.1 Choice and Preparation of Specimen and XRD Experiment ............................68

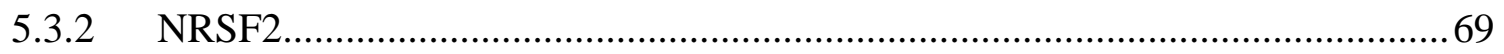

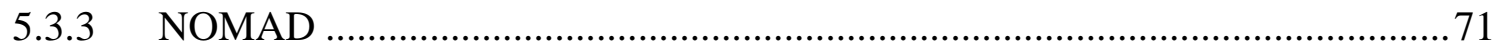

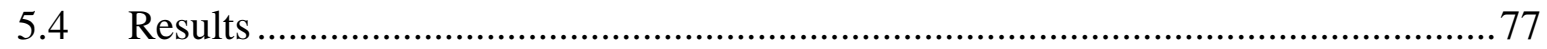

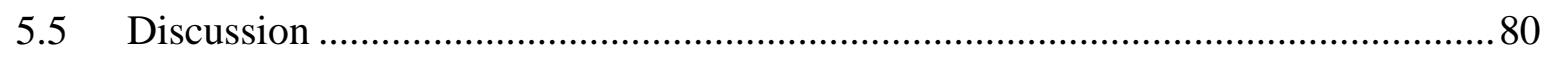

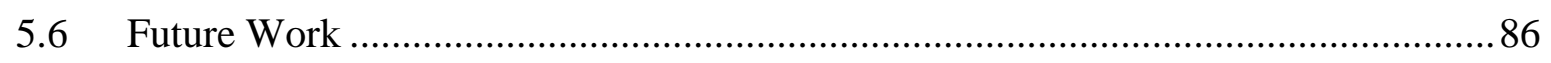

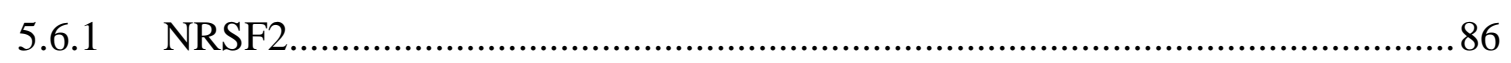

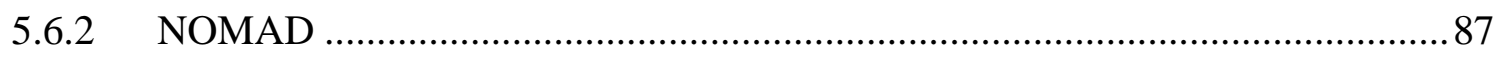

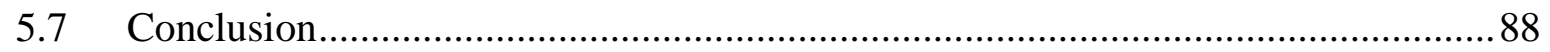

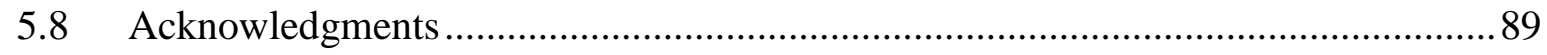

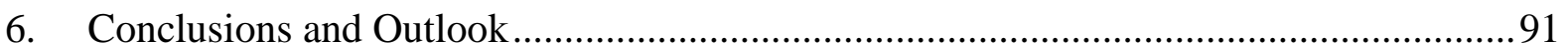

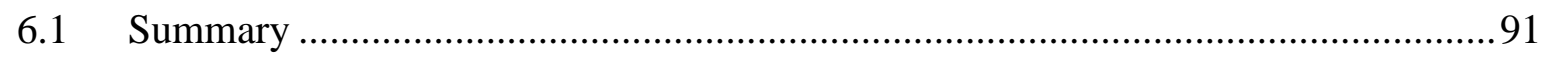

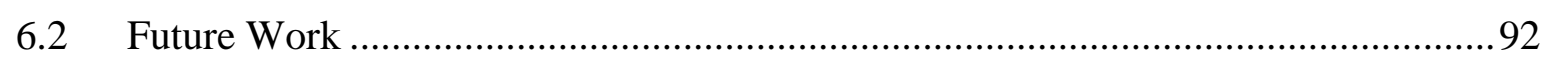

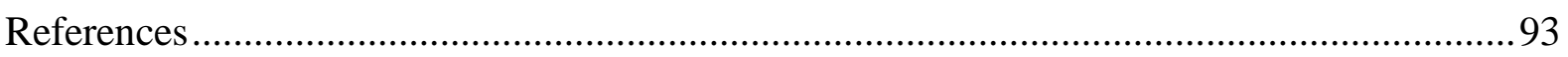




\section{Table of Figures}

Figure 1.1 "Earing" of aluminum cans. Taken from (Engler and Randle, 2010). 1

Figure 1.2 A single grain orientation.

Figure 1.3 Randomly textured sample. The white arrows represent the normal vectors to the (001) planes (i.e. [001] directions).

Figure 1.4 Sample with (001) planes parallel to $x-y$ plane. The orange arrows represent the preferred orientation.

Figure 1.5 Diffraction from (a) single crystal, (b) randomly textured polycrystal and (c) textured polycrystal.

Figure 1.6 Schematic of the pole figure measurement technique: (a) grains in a textured polycrystalline specimen, (b)-(d) rotations of the specimen to bring each set of grains into the diffraction condition.

Figure 1.7 Schematic of the energy-dispersive measurement technique. Incident and diffracted vectors are represented by the solid gray and black dashed arrows, respectively..... 9

Figure 1.8 Ambiguity of the PDF. The specimen and crystal coordinates are $\boldsymbol{X}, \boldsymbol{Y}, \boldsymbol{Z}$ and $\boldsymbol{a}, \boldsymbol{b}, \boldsymbol{c}$, respectively. The crystal and specimen directions are $\boldsymbol{h}$ and $\boldsymbol{r}$, respectively.............11

Figure 1.9 De la Vallee Poussin kernel with various half-widths..........................................15

Figure 2.1 Bragg-Brentano reflection geometry, with rotation axes relevant for texture........22

Figure 2.2 Pole figure angles with respect to goniometer rotations. ....................................23

Figure 2.3 Coverage of equal angle (left) vs. proposed grid scheme. ...................................25

Figure 2.4 Experimental pole figures for $5^{\circ} \times 5^{\circ}$ grid (top) and ring grid............................27

Figure $2.5\{111\}$ Pole figures recalculated from the ODF for (from left) $5^{\circ} \times 5^{\circ}$, ring grid and

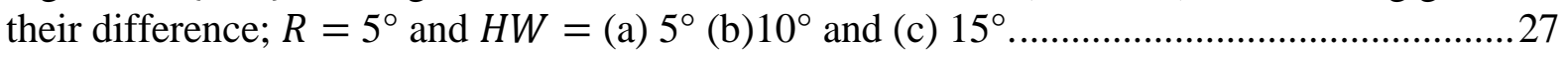

Figure 2.6 Experimental $\alpha=35^{\circ}$ curves for each of the two methods...............................28

Figure $2.7 \mathrm{ODF}$ fits to $\alpha=35^{\circ}$ curves for (a) $5^{\circ} \times 5^{\circ}$ and (a) ring grid.............................28

Figure 2.8 Comparison of fits to $\alpha=35^{\circ}$ curves for (a) $R=5^{\circ}, H W=5^{\circ}$ and (b) $R=5^{\circ}$,

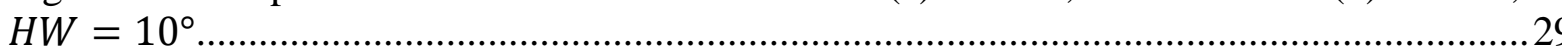

Figure 2.9 Sections $\left(\varphi_{2}=0-90^{\circ}\right.$ in steps of $\left.5^{\circ}\right)$ of the $5^{\circ} \times 5^{\circ}$ grid ODF (orthotropic specimen symmetry) computed using $R=5^{\circ}, H W=10^{\circ}$.

Figure 2.10 Sections $\left(\varphi_{2}=45,65\right.$ and $\left.90^{\circ}\right)$ of the ODF (orthotropic specimen symmetry) computed using $R=5^{\circ}, H W=10^{\circ}$ for (a) $5^{\circ} \times 5^{\circ}$ grid and (b) ring grid. Common FCC rolling and recrystallization texture components are labeled. 
Figure 3.1 Diffraction geometry. Note that $\boldsymbol{Q}_{\mathbf{1}}$ represents diffraction from planes with their poles aligned near the compression axis.

Figure 3.2 (a) Difference plot for all 72 spectra, (b) fit to experimental data for radial pattern, (c) same as (b) for axial pattern. The black dots are experimental data, and the red curve is the Rietveld fit. From top to bottom starting immediately below horizontal axis: $\alpha-M g$ peak ticks,

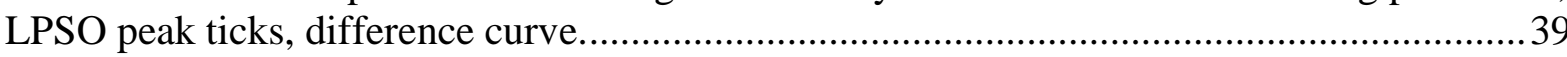

Figure 3.3 Agreement to experimental data $R_{w p}$ (equation 1.13) vs. strain..........................40

Figure 3.4 Fit to experimental data vs. strain: (a) initial pattern (no strain), (b) intermediate

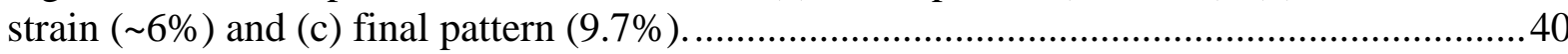

Figure 3.5 Initial texture of (a) $\alpha-\mathrm{Mg}$ phase, and (b) LPSO phase. The ED (compression axis) is in the center of the pole figure.

Figure 3.6 Evolution of texture as a function of strain and stress for (a) $\alpha-\mathrm{Mg}$ and (b) LPSO. Open symbols and dashed lines are axial (center of pole figure), closed symbols and solid lines are radial (periphery).

Figure 3.7 Basal reflection intensity vs. polar angle for initial and final texture of (a) $\mathrm{Mg}$ and (b) LPSO phases.

Figure 4.1 Attenuation relative to the initial intensity of $\mathrm{Cu} \mathrm{K \alpha}$, Mo $\mathrm{K} \alpha$ and neutron radiation in depleted $\alpha$-uranium, as a function of path length through the material. The path length required for diffraction is considerably longer than the depth into the surface

Figure 4.2 Polarized optical micrographs of the $\alpha$-uranium foil for (a) view along RD and (b) view along ND, showing equiaxed $10 \mu \mathrm{m}$ diameter grains and bands of precipitates (noted with black arrows) along the rolling direction that are related to carbon impurities.

Figure 4.3 Pole figures from the calculated ODF of the $\alpha$-uranium foil bulk texture measured by neutron diffraction, using (a) the full complement of 100 measured diffraction peaks or (b) only the (002), $\{111\}$, and $\{112\}$ peaks to compute the ODF.

Figure 4.4 Pole figures from the calculated ODF of the $\alpha$-uranium foil surface texture measured using $\mathrm{Cu} \mathrm{K} \alpha \mathrm{X}$-rays and the (002), $\{111\}$, and $\{112\}$ peaks.

Figure 4.5 Pole figures of the difference in MRD intensity between the surface (Figure 4.4) and bulk (Figure 4.3) texture measurements. The rolled foil's orthotropic sample symmetry has been imposed to smooth experimental variations in intensity and better clarify trends....59

Figure 4.6 Pole figures from the calculated ODF of the $\alpha$-uranium foil surface texture measured using Mo Ka X-rays and the (002), $\{111\}$, and $\{112\}$ peaks.

Figure 5.1 Detector coverage mapped onto the pole figure (at zero sample rotation) for (a) NOMAD and (b) HIPPO. Error! Bookmark not defined.

Figure 5.2 Geometry of pole figure measurement on NRSF2. The "detector" is 7 individual detectors that extend out of the page on a circular arc. 
Figure 5.3 The NOMAD detector suite, with active detectors shown in multi-color. The instrument coordinate system is $+\mathrm{Z}=$ blue (beam direction), $+\mathrm{Y}=$ green, $+\mathrm{X}=$ red. The origin is the sample location.

Figure 5.4 NOMAD pole figure coverage: (a) binned by detector tube, with instrument coordinates; (b) texture binning scheme (65 bins), $\omega=0^{\circ}$ (blue); (c) $\omega=0^{\circ}, 55^{\circ}$ (red), $90^{\circ}$ (green), $155^{\circ}$; (d) same as (c) with no $\omega^{\circ}=0^{\circ}$ and (e) same as (c) with reduced coverage........74

Figure 5.5 Pole figures (units: MRD) recalculated from the ODF for (a) XRD, annotated; (b) XRD; (c) NRSF2; NOMAD: (d) E-WIMV $5^{\circ}$ resolution and (e) harmonic. the annotations in (a) are the FCC rolling and recrystallization "ideal" texture components copper $\boldsymbol{\Delta}$, brass $\mathbf{\nabla}, \mathrm{S}$ $\checkmark$, Goss $\bullet$, and cube

Figure 5.6 Maximum ODF intensity in constant sections along the beta fiber skeleton line. Orthotropic (point group $\mathrm{mmm}$ ) specimen symmetry is assumed.

Figure 5.7 Maximum ODF (orthotropic specimen symmetry) intensity in constant sections along the beta fiber skeleton line for (a) XRD results at mid-plane ("original"), 3/8-plane and their arithmetic average and (b) NRSF2 with two possible reduction schemes.

Figure 5.8 NOMAD detector coverage: (a) Current (b) Empty panels (red) included (c) Proposed additions included. (d) Binned, 0 and $90^{\circ}$ (red) with proposed additions included. 88 


\section{List of Tables}

Table 2.1 Volume fraction of several FCC rolling and recrystallization components and $J_{O D F}$ for both methods with two $H W$ choices (orthotropic specimen symmetry).

Table 5.1 Volume fraction (in \%) of several FCC rolling and recrystallization components and $J_{O D F}$ for each of the 4 ODFs (orthotropic specimen symmetry). The ratio "Roll/Rec." was computed by dividing the sum of the first three rows (rolling components) by the sum of rows

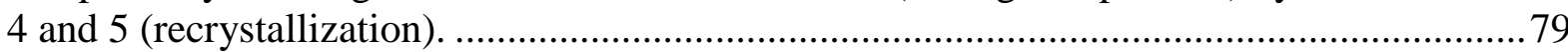

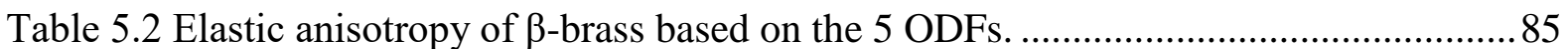




\section{Introduction}

\subsection{Motivation}

Texture is important because (a) it has a significant effect on material properties and (b) it develops due to processing and thus is extremely common in material systems of interest to engineers. In particular, texture is related to the anisotropy, or dependence on direction, of material properties. For example, the ability of aluminum alloys to shape properly during "deep-drawing" (e.g. for beverage cans) is closely tied to their having a specifically balanced texture. Otherwise, their thermomechanical response will be anisotropic, causing "earing" around the top of the can during drawing, as shown below (Engler and Randle, 2010). This can jam the drawing equipment, resulting in costly production downtime and lower yield.

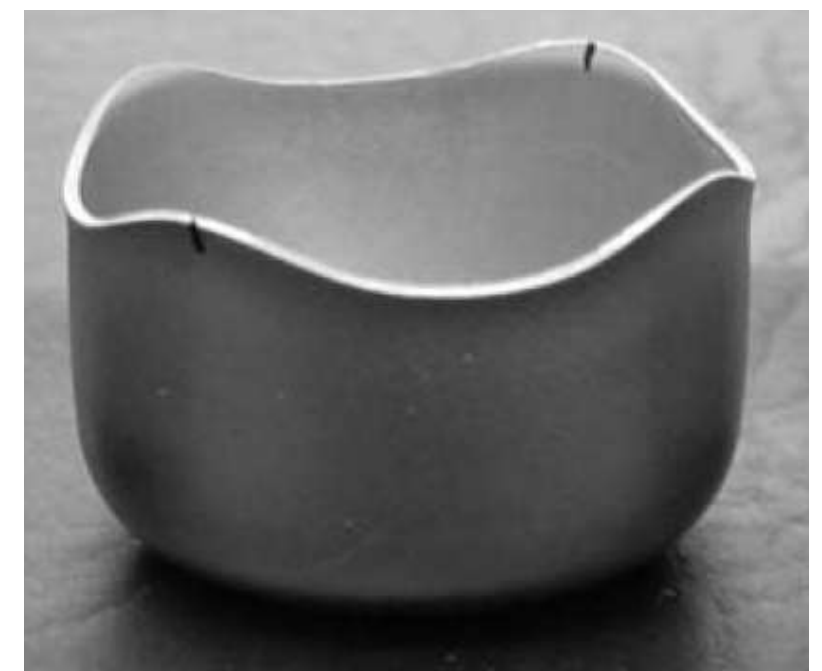

Figure 1.1 "Earing" of aluminum cans. Taken from (Engler and Randle, 2010).

Perhaps the most commonly studied class of properties in the context of texture effects is mechanical properties. One example is the effect of texture on polycrystalline elastic response, which is known to play a significant role in the mechanical behavior of metastable $\beta$-Ti alloys (Helstroffer et al., 2016). Texture also effects thermal properties and thus is important for the study of high temperature ceramics like $\mathrm{LaPO}_{4}$ monazite, which are used in structural applications (Du et al., 2010) and Zirconia, which is used for high temperature coatings (Heydt et al., 2001). Bulk magnetic properties such as core loss and relative permeability are known to vary depending on texture in 
electrical steels, which are important for high-efficiency motors and drives (Ghosh et al., 2014). Pavithra et al. (2015) enhanced the electrical and mechanical properties of copper foils, which are used extensively in electronic device applications, by carefully tuning the texture via Pulse Reverse Electrodeposition (PRED).

To expand upon the second point, texture develops and/or changes whenever a material is deformed appreciably, undergoes a solid state phase transformation, or recrystallizes upon cooling (Engler and Randle, 2010). It can also develop as a result of solidification processes such as casting (Sturcken and Walter, 1974) or thin film growth (Budai et al., 2003). In the context of materials science, texture is the result of intentional processing, whereas in geology, texture results from "processing" beneath the Earth's crust. Thus, studying how texture changes with processing gives an understanding of the unknown history of material (in geology) or gives insight as to how to better process materials to optimize certain properties (in materials science). A survey of some interesting problems in geology is given in Ivankina and Matthies (2015). Gómez Barreiro, Wenk, and Vogel (2015) measured and analyzed mylonitic anorthosite minerals from the lower crust near Quebec, Canada, to better understand geothermal processes in that region. Understanding the additive manufacturing of metals is a problem of particular importance and popularity in materials engineering, and strong textures quite commonly develop as a result of such processing. Raghavan et al. (2016) developed a novel melt strategy for the additive manufacturing of Ni-based super-alloy IN718 based on the goal of weakening a texture that leads to undesirably sharp anisotropy in the mechanical properties.

In many cases, the two points are inter-related and it is desirable to study how the texture evolves during processing, which in turn effects the material's properties and thus its later response to processing, which again affects the next stage of textural evolution, and so on. In this way, texture fits well into the classic processing-structure-properties paradigm of materials science and engineering. As an example, crystal plasticity models typically include the initial texture as an input parameter but also seek to model the evolution of texture as the material is loaded, heated or both (Tahreen et al., 2016). 
With this motivation, texture will be defined in the next section and explored in great detail throughout this thesis.

\subsection{What is Texture?}

A material is called crystalline if its atoms are arranged in a repeating, space-filling pattern*. This can be true throughout the material, as is the case for a single crystal, but most crystalline solids of interest to engineers are polycrystalline. This means they are made up of many separate single crystals, or "grains," that generally vary in size, shape and orientation. All three of these microstructural variables are important in the study of materials, but it is the variation in grain orientations in a material that gives rise to texture.

In simple terms, a single grain's orientation is defined by how it is rotated in space with respect to some set of reference coordinates, usually defined by the sample geometry. For example, for a rolled sheet, we generally define the sample coordinates by the rolling geometry ${ }^{\dagger}$, i.e. the rolling direction (RD) is designated as $\boldsymbol{X}$, the transverse direction (TD) $\boldsymbol{Y}$, and the normal direction (ND) $\boldsymbol{Z}$. A particular grain's orientation is defined by the angles between its crystallographic axes (the crystal coordinates) and the sample coordinate frame (or vice versa). Figure 1.2 shows a schematic representation of an arbitrary grain orientation. In this case, the crystallographic axes [100], [010] and [001] are all inclined with respect to the sample frame $\boldsymbol{X}, \boldsymbol{Y}$, and $\boldsymbol{Z}$, and the inclination can be described as an orientation $^{\ddagger}$. Most polycrystalline materials have a large number of grains, each with their own orientation. Texture describes which of these orientations exist in the sample and in what proportions.

\footnotetext{
* Quasicrystals are an exception, see (Levine and Steinhardt, 1984).

${ }^{\dagger}$ In general, the sample coordinates can be chosen to correspond to any convenient axes relative to the sample geometry or processing. In some cases, no such axes exist, in which case the choice is arbitrary.

* The most common description of orientations is by the Euler angles, and the numerous conventions for how these are defined are given in Engler and Randle 2010, Chapter 2.6.
} 


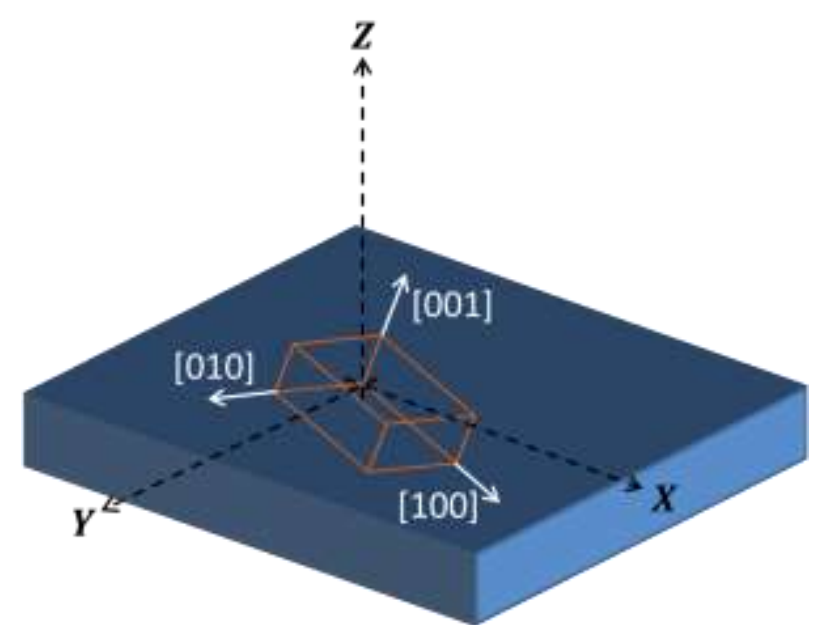

Figure 1.2 A single grain orientation.

A polycrystalline material is said to be "randomly textured" or is said to have "no texture" if all possible grain orientations are represented in the material in equal amounts, i.e. there is no particular orientation that appears more often than the others. Conversely, a material is said to be textured (or to have preferred orientation) if one or multiple orientations are represented in a greater proportion than the others. A texture is "strong" if the sample has very high volume fractions of certain orientations and "weak" if it is closer to random.

To make this more concrete, consider a small rectangular sample of polycrystalline metal with an orthorhombic crystal structure, say $\alpha$-uranium (Anderson et al., 1982). If the sample were randomly textured, the (001) planes, for example, of the individual crystallites would be pointed in all different directions, with no one direction preferred over the others. Figure 1.3 shows this schematically.

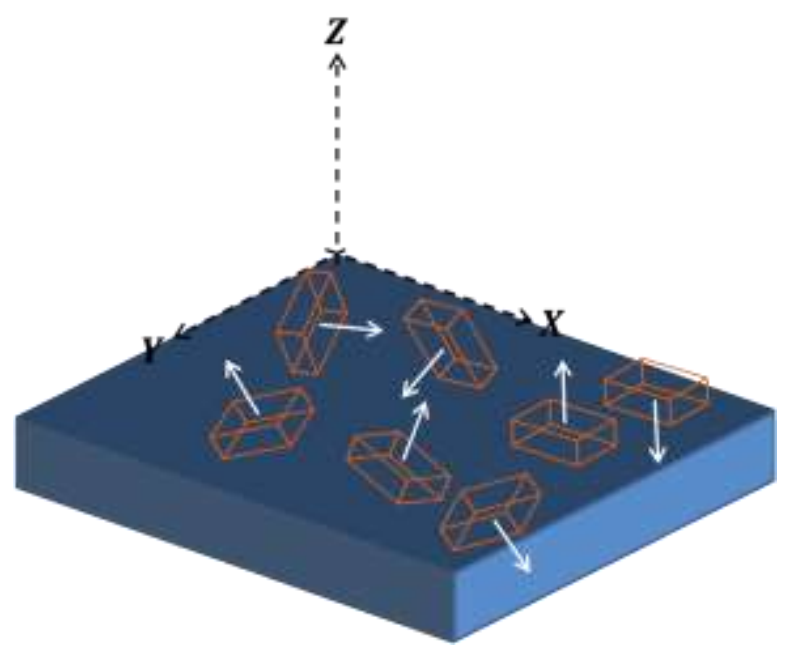


Figure 1.3 Randomly textured sample. The white arrows represent the normal vectors to the (001) planes (i.e. [001] directions).

However, some volume fraction of the grains, say 63\%, might have their (001) planes oriented parallel to the top (x-y) plane of the block (i.e. the [001] directional axes parallel to the $\mathrm{z}-$ axis). This particular orientation would then be a preferred one, and so the sample is textured. Figure 1.4 shows this schematically.

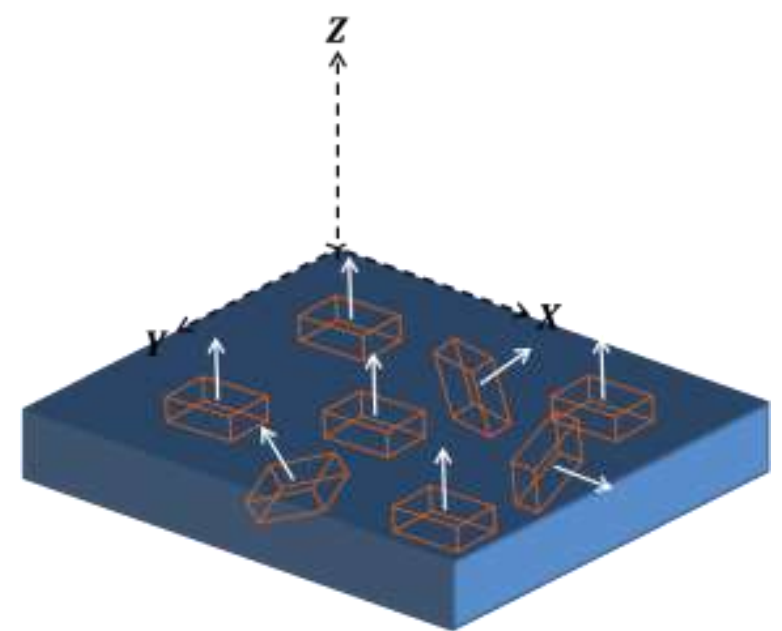

Figure 1.4 Sample with (001) planes parallel to $x-y$ plane. The orange arrows represent the preferred orientation.

In some applications, it is sufficient to know only the statistics of texture, that is, the volume fraction of each orientation over the entirety of the sample volume. This is sometimes called "macrotexture" (Engler and Randle, 2010). In other cases, it is important to determine not only the overall texture of the solid, but also how the orientations are distributed spatially throughout the material at the micro-scale. This is often called "microtexture" analysis.

\subsection{Diffraction and Texture}

Though they may differ in how the information is collected or conveyed, all modern methods for texture analysis exploit the same principles of diffraction. Incident radiation of wavelength $\lambda$ diffracts from lattice planes $\{h k l\}$ with spacing $d_{h k l}$ when the Bragg condition is met:

$$
n \lambda=2 d_{h k l} \sin (\vartheta),
$$

where $n$ is the order of diffraction (generally $n=1$ ) and $2 \vartheta$ is denoted the Bragg angle. The intensity of diffracted radiation spikes when this condition is met (diffraction peaks) and is nearly zero 
otherwise (background). Diffraction experiments measure the intensity as a function of $\vartheta$ with $\lambda$ fixed or vice versa, in order to determine the lattice spacings $d_{h k l}$.

In a typical diffraction experiment utilizing monochromatic radiation, the incident radiation is represented by the vector $\boldsymbol{k}_{i}$, the diffracted radiation by the vector $\boldsymbol{k}_{f}$, and the diffraction vector $\boldsymbol{Q}$ by the vector quantity $\boldsymbol{k}_{f}-\boldsymbol{k}_{i}$ (Figure 1.5). For a single crystal, diffraction is observed only when the sample is oriented properly in 3-D space such that $\boldsymbol{Q}$ is parallel to the reciprocal lattice vector $\boldsymbol{G}_{h k l}$, which is normal to the plane $\{h k l\}$ and has length inversely proportional to the inter-planar spacing $d_{h k l}$ (Figure 1.5(a)). 1.1In contrast, a polycrystalline material with a random distribution of orientations (e.g. a powder sample) diffracts in Debye-Scherrer cones, each related to a given lattice plane $\{h k l\}$ and corresponding to full rotation of the diffracted beam about the incident beam, with uniform intensity along the circular edge of the cone (Figure 1.5(b)). A textured polycrystal can be thought of as lying between these two extremes: it also diffracts in cones, but the intensity can vary as a function of location along the edge of the cone. This results from the fact that textured polycrystals by definition contain more crystallites oriented in certain directions than others, causing the contribution to the diffracted intensity to depend on the sample orientation (Figure 1.5(c)).

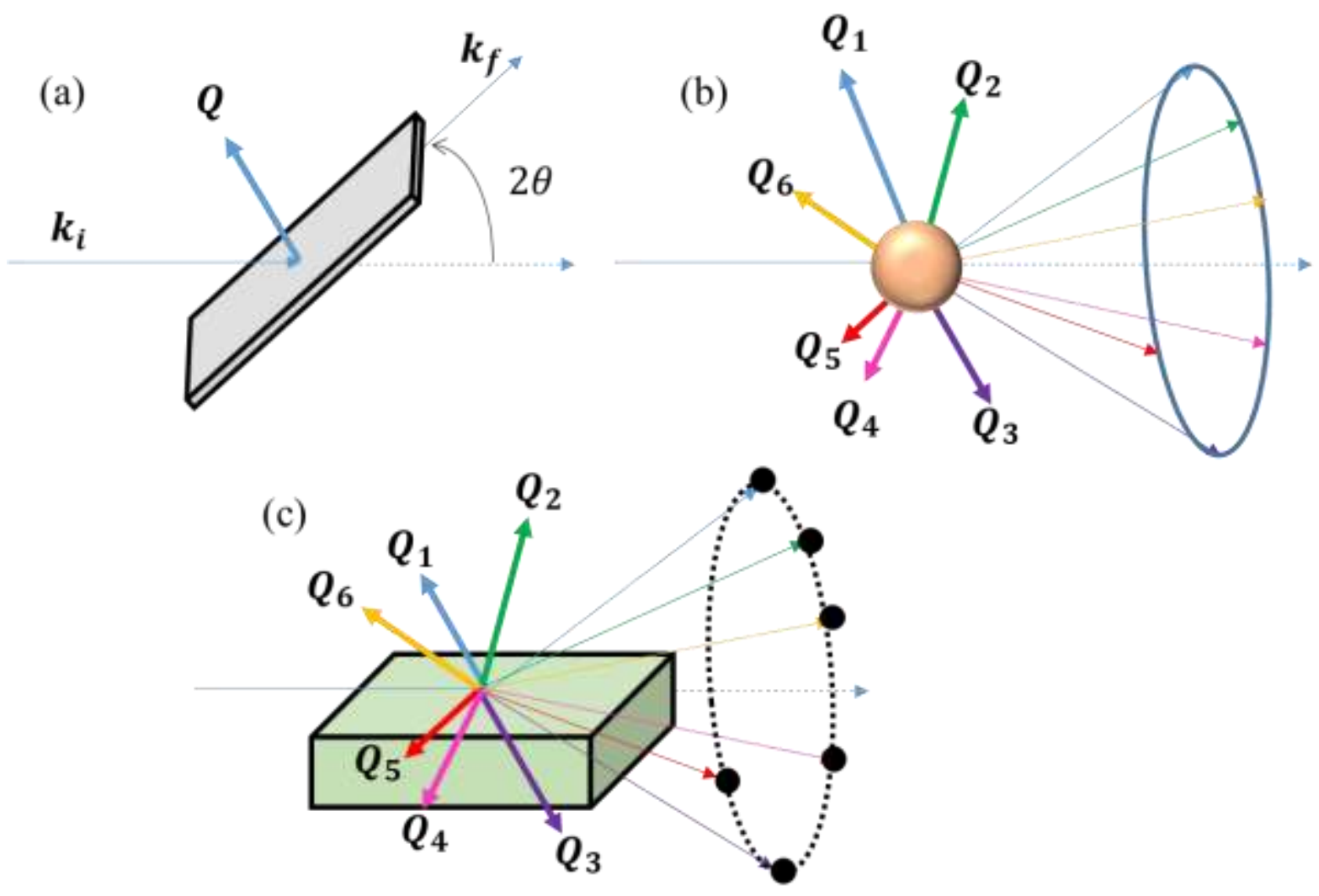


Figure 1.5 Diffraction from (a) single crystal, (b) randomly textured polycrystal and (c) textured polycrystal.

Texture is analyzed via the diffraction experiment by determining what volume fraction of crystallites will diffract in a given orientation, i.e. by manipulating the sample so that different $\boldsymbol{Q}$ 's meet the diffraction condition and measuring the intensity. The set of all possible orientations of all $Q$ 's is known as orientation space (or Euler space if represented by the Euler angles), which is 3-D and thus difficult to visualize. The volume fraction of each orientation is reported by the orientation distribution function (ODF), and computing the ODF is the goal of quantitative texture analysis.

Smaller and easier to visualize regions of orientation space are given by the pole figure or inverse pole figure, which are common (2-D) graphical representations for texture. The pole figure represents a set of orientations of the sample coordinates around a given $\boldsymbol{G}_{h k l}$ (e.g. $\boldsymbol{G}_{001}$, the vector normal to the (001) planes), as defined by the diffraction condition, and the inverse pole figure represents a set of orientations of the crystal coordinates around a given vector in the sample coordinates (e.g. the ND in a rolled sheet of metal).

\subsection{Methods for Texture Analysis}

\subsubsection{Philosophical Differences}

The differences between methods for texture analysis ultimately originate from the differences between diffraction experiments. In view of Bragg's law (equation 1.1), one can measure the intensity as a function of $\vartheta$ with $\lambda$ fixed, or as a function of $\lambda$ with $\vartheta$ fixed. The former is known as "angular-dispersive" and employs monochromatic source radiation and typically a single (movable) detector, while the latter is called "energy (or wavelength)-dispersive" and employs polychromatic radiation and many fixed-location (or one position-sensitive) detectors.

For angular-dispersive methods, the typical approach involves setting a single detector at the Bragg condition for a given lattice plane $\{h k l\}$ and rotating the sample such that $\boldsymbol{Q}$ maps out a hemisphere with respect to the initial sample orientation. This is shown schematically in Figure 1.6, where $\boldsymbol{Q}$ is fixed while the sample is rotated and tilted to bring grains with different orientations into the diffraction condition. The initial sample orientation is represented by the axes $\boldsymbol{X}, \boldsymbol{Y}$, and $\boldsymbol{Z}$. This is 
the procedure for measuring a pole figure, which is merely a projection (e.g. stereographic (Wulff) and equal area (Schmidt) are common; see Engler and Randle 2010) of the hemisphere onto a flat surface. This is the classic technique for texture measurement and will be explored in Chapters 2, 4 and 5. It is also possible to measure texture with monochromatic radiation by imaging (2-D slices of) Debye cones directly with area position-sensitive detectors (PSDs) or Laue (e.g. CCD) cameras (Garces et al., 2015a; Ischia et al., 2005; Lonardelli et al., 2005; Lutterotti et al., 2007). This is typically done at a synchrotron, where the $\mathrm{x}$-ray energy is large so that the cones are narrow (small Bragg angles). This more recently developed technique will be explored in Chapter 3.
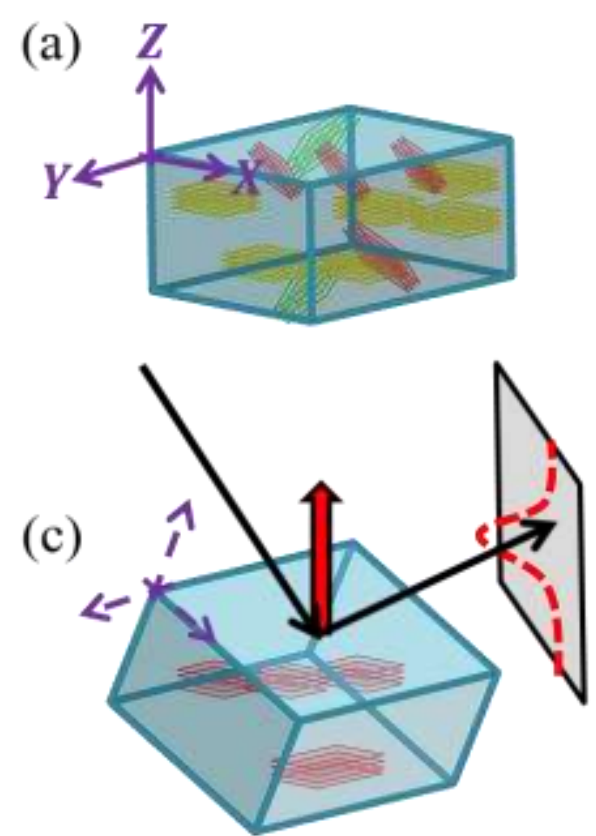
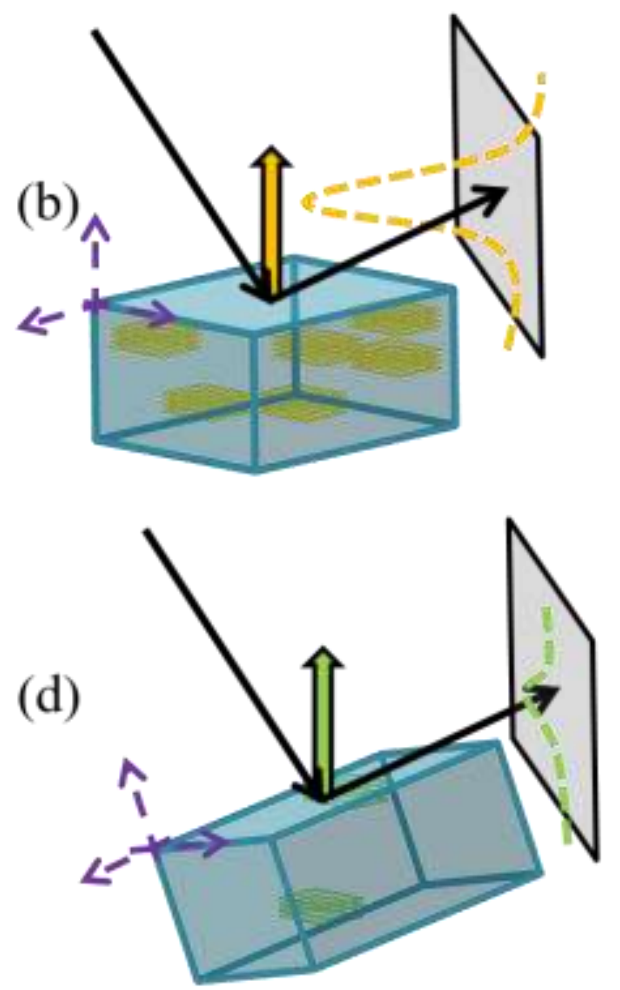

Figure 1.6 Schematic of the pole figure measurement technique: (a) grains in a textured polycrystalline specimen, (b)-(d) rotations of the specimen to bring each set of grains into the diffraction condition.

Energy-dispersive techniques typically employ polychromatic source radiation and a detection technique that allows for both the energy and the intensity of diffracted radiation to be determined. The most obvious benefit of this technique for texture measurement is that $\theta$ is fixed so the detector can remain stationary and still capture a number of diffraction peaks, depending on the energy range of the detected radiation (equation 1.1). If a sufficient number of diffraction peaks are 
available, it may be possible to directly measure an inverse pole figure using this technique (Stoica et al., 2014). More recently, this has been exploited by using multiple fixed detectors, so that many "sample orientations" or inverse pole figures (corresponding to the different detector locations) can be measured at once. This is shown schematically in Figure 1.7. For XRD, energy-dispersive measurement requires a polychromatic x-ray source and a detector that can directly discriminate between x-ray photons of different energy levels.

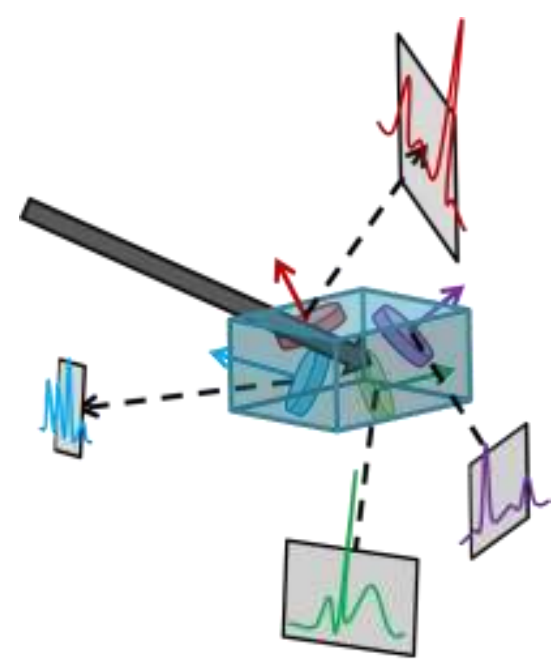

Figure 1.7 Schematic of the energy-dispersive measurement technique. Incident and diffracted vectors are represented by the solid gray and black dashed arrows, respectively.

The neutron analogy to energy-dispersive XRD is time-of-flight (TOF) neutron diffraction. Polychromatic neutrons are used as the source, but direct discrimination of neutron energies is not currently possible. Instead, the velocity $v$ of neutrons is determined from the time $t$ it takes for them to traverse the fixed distance $L$ from source to sample by $v=\frac{L}{t}$. Then the wavelength can be computed as follows (Hutchings et al., 2005):

$$
\lambda=\frac{h t}{m L} .
$$

Neutrons are counted by the detector(s), and this signal is fed to a time analyzer that is synchronized with the neutron beam pulse from the source (Los Alamos National Laboratory, 2015). The time analyzer bins the neutron counts by TOF and records the intensity value for each bin. For better statistics, this is repeated over many pulses, and the results are summed. This results in a diffraction pattern with intensity vs. $t$ (i.e. $\lambda$ or $d$ ), without having to move the source or detector. In 
other words, $\lambda$ is varied with $\vartheta$ fixed (equation 1.1). TOF neutron diffraction methods will be explored in great detail in Chapters 4 and 5.

A final method for texture analysis is the direct measurement of individual grain orientations, which can be performed with either electrons or, more recently, high-energy x-rays. The former involves the use of electron backscatter diffraction (EBSD) in a scanning electron microscope (SEM), while the latter is typically performed at a synchrotron. For microtexture analysis, so-called "orientation maps" can be produced where the individual grain orientation information is superposed onto a micrograph (Wrights et al., 1991). For macrotexture analysis, a large number of orientations are averaged so that the data approximates what is recorded in a more conventional x-ray or neutron diffraction experiment.

More experimental details and differences of each technique will be given in section 1.4.5 and later chapters. The quantitative analysis of data from the three categories of texture experiment will now be explored.

\subsubsection{Pole Figure Inversion}

The inherent differences between the two measurement approaches results in fundamental differences in the way the data is analyzed for texture. Historically, the most common analysis approach has been pole figure inversion: a number of pole figures are collected, and the orientation distribution function (ODF), the standard quantitative descriptor of texture, is computed. This problem will now be explored in detail.

An orientation can be defined by a rotation matrix $\boldsymbol{g}$ that rotates the specimen coordinates $\boldsymbol{K}_{\boldsymbol{S}}=(\boldsymbol{X}, \boldsymbol{Y}, \boldsymbol{Z})$ into the crystal coordinates $\boldsymbol{K}_{\boldsymbol{C}}=(\boldsymbol{a}, \boldsymbol{b}, \boldsymbol{c})$ via $\boldsymbol{K}_{\boldsymbol{C}}=\boldsymbol{g} \boldsymbol{K}_{\boldsymbol{S}}$. In other words, the crystal's axes are oriented away from the fixed reference coordinate system (here $\boldsymbol{K}_{\boldsymbol{S}}$ ), and the deviation is given by $\boldsymbol{g}^{*}$. Any direction in either the crystal $(\boldsymbol{h})$ or the specimen $(\boldsymbol{r})$ system can be represented as integer multiples of the basis vectors $\boldsymbol{K}_{\boldsymbol{C}}$ and $\boldsymbol{K}_{\boldsymbol{S}}$, respectively,

$$
\boldsymbol{h}=\left[\begin{array}{l}
h \\
k \\
l
\end{array}\right] \cdot \boldsymbol{K}_{\boldsymbol{C}}, \boldsymbol{r}=\left[\begin{array}{c}
u \\
v \\
w
\end{array}\right] \cdot \boldsymbol{K}_{\boldsymbol{S}} .
$$

\footnotetext{
${ }^{*}$ Note that if $\boldsymbol{g}$ is the identity matrix, then $\boldsymbol{K}_{\boldsymbol{S}}$ and $\boldsymbol{K}_{\boldsymbol{C}}$ coincide.
} 
Because the crystal has symmetry and thus crystallographically equivalent directions, orientations can be symmetrically equivalent: if there exists a symmetry element $\boldsymbol{q}$ in the Laue class of the crystal such that $\boldsymbol{g} \boldsymbol{q}=\boldsymbol{g}^{\prime}$, then $\boldsymbol{g}$ and $\boldsymbol{g}^{\prime}$ are symmetrically equivalent. The ODF is given by $f(\boldsymbol{g})$, which is simply a real-valued function describing (in physical terms) the number of crystallites with orientation $\boldsymbol{g}$ in the specimen. Because orientations, as described in this way, are not directly observable by experiment (i.e. diffraction), it is necessary to also define them based on poles (or plane normal vectors) in the crystal, which are described by crystal directions $\boldsymbol{h}$ and their orientation relationship with specimen directions $\boldsymbol{r}$. However, defining only $(\boldsymbol{h}, \boldsymbol{r})$ leaves an extra degree of freedom with respect to the description $\boldsymbol{g}$, since a given $\boldsymbol{h}$ can lie parallel to $\boldsymbol{r}$ in an infinite number of ways (though of course some may be crystallographically equivalent). This ambiguity is shown schematically in Figure 1.8: the crystal can be rotated arbitrarily about $\boldsymbol{h}$ while still maintaining $\boldsymbol{h} \| \boldsymbol{r}$.

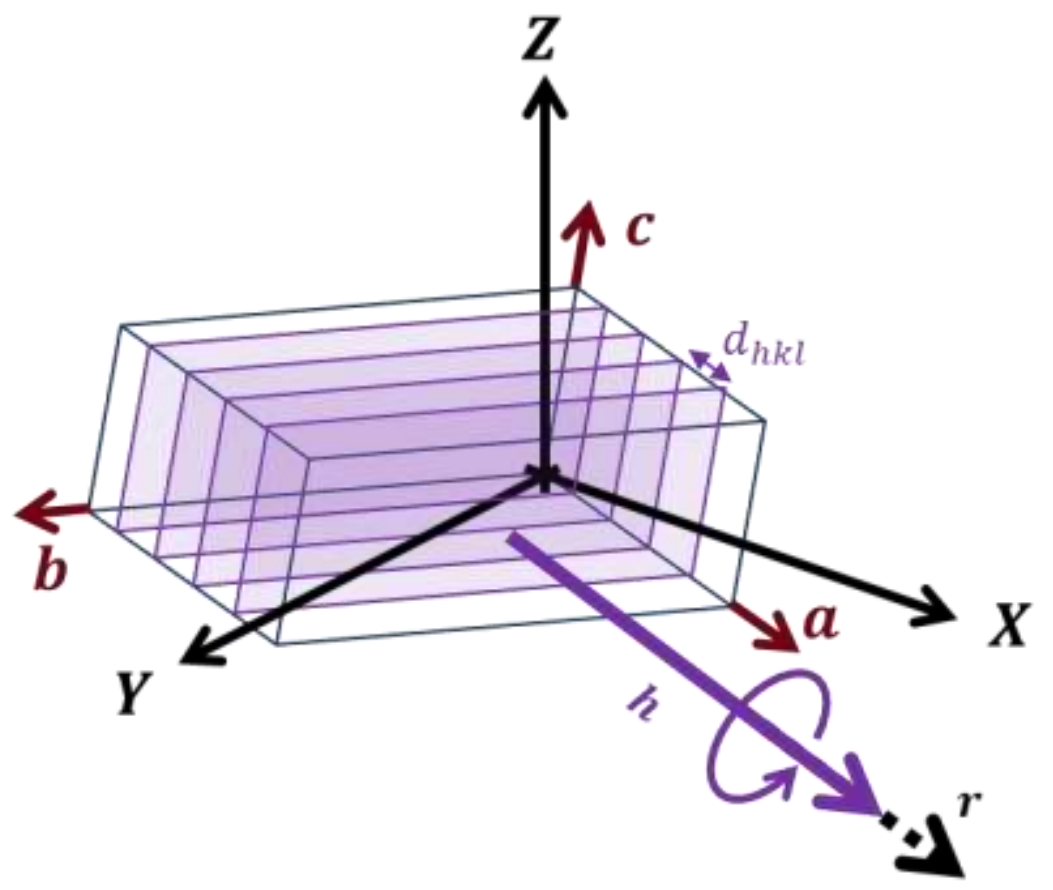

Figure 1.8 Ambiguity of the PDF. The specimen and crystal coordinates are $(\boldsymbol{X}, \boldsymbol{Y}, \boldsymbol{Z})$ and $(\boldsymbol{a}, \boldsymbol{b}, \boldsymbol{c})$, respectively. The crystal and specimen directions are $\boldsymbol{h}$ and $\boldsymbol{r}$, respectively.

The pole distribution function (PDF) is the 2-D vector analogy to the ODF and is given by $P(\boldsymbol{h}, \boldsymbol{r})$, a real-valued function describing the volume fraction of crystallites in the specimen with their lattice plane normal (or crystal direction) $\boldsymbol{h}$ oriented parallel to some specimen direction $\boldsymbol{r}$. The 
problem of pole figure inversion is summarized by the relationship between the PDF and ODF (Engler and Randle, 2010):

$$
P(\boldsymbol{h}, \boldsymbol{r})=\frac{1}{2 \pi} \int_{0}^{2 \pi} f(\boldsymbol{g}) d \gamma
$$

where $\gamma$ is the angle through which $\boldsymbol{h}$ can be rotated while remaining parallel to $\boldsymbol{r}$ (the extra rotational degree of freedom described in Figure 1.8). Typically, graphical representations of texture (e.g. plots of $P(\boldsymbol{h}, \boldsymbol{r})$ or sections of $f(\boldsymbol{g}))$ are shown in units of multiples of a random distribution (MRD), where the normalization is such that every orientation for a randomly textured sampled has 1 MRD (Engler and Randle, 2010). Equation 1.4 is referred to as the fundamental equation of ODF computation, and the process of pole figure inversion aims at solving it.

\subsubsection{The Harmonic Method}

The conventional pole figure inversion method is known as the series expansion or harmonic method because of its use of spherical harmonic functions. Bunge (1965) and Roe (1965) are credited with independently developing the formulation for quantitative texture analysis. The standard spherical harmonic functions $\kappa_{l}^{n}(\boldsymbol{r})$ and $\kappa_{l}^{m}(\boldsymbol{h})$ are together proposed as a solution to $P(\boldsymbol{h}, \boldsymbol{r})$ (the PDF), and the generalized spherical harmonic functions $\tau_{l}^{m n}(\boldsymbol{g})$ are proposed as a solution to $f(\boldsymbol{g})$ (the ODF). Substituting into equation 1.4 and accounting for normalization of the PDF yields (Engler and Randle, 2010):

$$
\left(\frac{2}{2 l+1}\right) \cdot \overline{\kappa_{l}^{m}}(\boldsymbol{h}) \cdot \kappa_{l}^{n}(\boldsymbol{r})=\frac{1}{2 \pi} \int_{0}^{2 \pi} \tau_{l}^{m n}(\boldsymbol{g}) d \gamma
$$

where the bar represents the complex-conjugate.

In analogy to Fourier analysis, the ODF is now expanded in a series of the functions $\tau_{l}^{m n}(\boldsymbol{g})$ :

$$
f(\boldsymbol{g})=\sum_{l=0}^{L_{\max }} \sum_{m=-l}^{l} \sum_{n=-l}^{l} C_{l}^{m n} \tau_{l}^{m n}(\boldsymbol{g})
$$

where the $C_{l}^{m n}$ are the series coefficients. In words (and just like in Fourier analysis), the unknown ODF is represented by a superposition of known functions, whose weights are given by the series coefficients. The PDF can also be expanded:

$$
P(\boldsymbol{h}, \boldsymbol{r})=\sum_{l=0}^{L} \sum_{n=-l}^{l} D_{l}^{n} \kappa_{l}^{n}(\boldsymbol{r}), \text { where } D_{l}^{n}=\left(\frac{4 \pi}{2 l+1}\right) \sum_{m=-l}^{l} C_{l}^{m n} \overline{\kappa_{l}^{m}}(\boldsymbol{h}) .
$$


The coefficients $C_{l}^{m n}$ are determined by fitting the coefficients $D_{l}^{n}$ to each of the $2 L+1$ experimental pole figures and solving the second part of equation 1.7. Finally, since the functions $\tau_{l}^{m n}(\boldsymbol{g})$ are known, the ODF $f(\boldsymbol{g})$ is computed via equation 1.6, and any PDF $P(\boldsymbol{h}, \boldsymbol{r})$ can be "recalculated" using equation 1.4. An exact solution is only possible when $L_{\max } \rightarrow \infty$, so in practice the analysis is performed numerically, with the associated error ("truncation error") determined by the specific value of $L_{\max }$ used in the computation. Generally the smaller the value of $L_{\max }$, the smoother the peaks in the predicted distribution (ODF), and so truncation error makes it difficult to model textures with exceedingly sharp features using the harmonic method (Engler and Randle, 2010). Increasing the value of $L_{\max }$ helps to alleviate this problem to a point but many software implementations have an upper limit to maintain feasible computational time and expense. One auxiliary benefit of the harmonic method is that polycrystalline tensor properties can be easily predicted using only the single crystal tensor and the coefficients $C_{l}^{m n}$ (Engler and Randle, 2010).

\subsubsection{Direct Methods}

Instead of attempting to fit continuous functions to the pole figure data, it is also possible to discretize the problem and directly derive values for the OD based on a number of PD's (the "F"'s are dropped because we are no longer referring to functions). First, we consider that we can represent the space of all possible orientations with a discrete grid of Euler angles (e.g. $0^{\circ}, 5^{\circ}, 10^{\circ}$, etc.) rather than allowing them to vary continuously. This means that instead of integrating corresponding portions of the ODF to yield a PDF, we can sum the OD "cells" to give a PD:

$$
P_{i}=\sum_{j=1}^{J} N_{i j} f_{j}
$$

where the $N_{i j}$ are the normalization constants and $J$ is the total number of OD cells. Since they do not rely on continuous functions to model features in orientation space, direct methods are more suitable than the harmonic method for modeling sharp textures (Matthies, 2002).

The WIMV method is one approach to solving equation 1.8 that uses a self-correcting iterative procedure (Matthies, 2002). An initial (0th iteration) guess for the OD is formulated by taking the geometric mean of all associated cells in the PD: 


$$
f_{0}(\boldsymbol{g})=N_{0} \prod_{k=1}^{K} \prod_{m_{k}=1}^{M_{k}}\left\{P_{\exp }\left(\boldsymbol{h}_{k}, \boldsymbol{r}_{m_{k}}\right)\right\}^{1 / K M_{k}}
$$

where $N_{0}$ is a normalization constant, $K$ is the total number of experimental pole figures, and $M_{k}$ is the multiplicity of the $k$ th pole. Now, assuming the current iteration is $n$, pole figures are recalculated from this OD via equation 1.8 and given by $P_{n}\left(\boldsymbol{h}_{k}, \boldsymbol{r}_{m_{k}}\right)$, which go into a correction factor $C_{n}$ :

$$
C_{n}=\frac{f_{0}(\boldsymbol{g})}{\prod_{k=1}^{K} \prod_{m_{k}=1}^{M_{k}}\left\{P_{n}\left(\boldsymbol{h}_{k}, \boldsymbol{r}_{m_{k}}\right)\right\}^{1 / K M_{k}}} .
$$

Now the guess for the next iteration is simply $f_{\mathrm{n}+1}(\boldsymbol{g})=f_{\mathrm{n}}(\boldsymbol{g}) C_{\boldsymbol{n}}$ and the process is repeated.

Now that the machinery has been presented, the simplest way to understand how the algorithm works is by walking through a few iterations. Equation 1.9 starts things off by guessing the ODF from the experimental pole figure data. Still in the $0^{\text {th }}$ iteration, we now re-calculate the pole figures $P_{0}\left(\boldsymbol{h}_{k}, \boldsymbol{r}_{m_{k}}\right)$ using equation 1.8, where the $f_{j}$ 's are simply the value of $f_{0}(\boldsymbol{g})$ in each OD cell $j$. Now we calculate the correction factor $C_{0}$ using equation 1.10 . In this case, the denominator is the geometric mean of the pole figures we just re-calculated in iteration 0 . If $P_{n=0}>P_{\text {exp }}$, i.e. the initial guess was too high, then $C_{0}<1$, and so $f_{1}(\boldsymbol{g})=f_{0}(\boldsymbol{g}) C_{0}$ will be smaller than $f_{0}(\boldsymbol{g})$. If it was too low, then $C_{0}>1$ and $f_{1}(\boldsymbol{g})>f_{0}(\boldsymbol{g})$. The beauty of the algorithm is that in either case, $C_{n}$ starts to approach 1 and the guesses start to converge to the experimental data.

Once we have computed $f_{1}(\boldsymbol{g})$, we start the 1 st iteration by re-calculating the pole figures $P_{1}\left(\boldsymbol{h}_{k}, \boldsymbol{r}_{m_{k}}\right)$ using equation 1.8 . Now we use this to compute $C_{1}$, which will hopefully be closer to 1 due to the correction to $f_{1}(\boldsymbol{g})$ at the end of the previous iteration. Still, if $C_{1}<1$ then $f_{2}(\boldsymbol{g})=$ $f_{1}(\boldsymbol{g}) C_{1}<f_{1}(\boldsymbol{g})$ and if $C_{1}>1$ then $f_{2}(\boldsymbol{g})=f_{1}(\boldsymbol{g}) C_{1}>f_{1}(\boldsymbol{g})$. Now, since $f_{2}(\boldsymbol{g})$ is closer to the "true" value of the OD, the re-calculated pole figures $P_{2}\left(\boldsymbol{h}_{k}, \boldsymbol{r}_{m_{k}}\right)$ will again be closer to $P_{\exp }$. The algorithm terminates when $\left(P_{n}-P_{\exp }\right)$ falls below a chosen convergence threshold, typically after about 10 iterations (Engler and Randle, 2010).

One of the drawbacks of WIMV is that it requires a regular grid $\left(\right.$ e.g. $\left.5^{\circ} \times 5^{\circ} \times 5^{\circ}\right)$ in orientation space, and so an interpolation routine must be included at each step to regularize experimental data that are not on such a grid (Lutterotti et al., 2004). This shortcoming was overcome 
by the introduction of the E-WIMV ("extended-WIMV") algorithm (Lutterotti et al., 2004), which can be used on data with arbitrary coverage of orientation space and is included in the MAUD program (along with WIMV). Some additional and less important differences between E-WIMV and WIMV are given in H. R. Wenk, Lutterotti, and Vogel 2003.

\subsubsection{The MTEX algorithm}

The MTEX algorithm, as implemented in the MATLAB texture toolbox of the same name (Bachmann et al., 2010), can be viewed as a combination of the harmonic and direct methods. Just as in the harmonic method, the ODF is estimated by linear combinations of functions, but now these functions are radially symmetric in orientation space. A suitable function (and the default in MTEX) is the bell-shaped de la Vallee Poussin kernel, as defined in Schaeben 1997. One way to control the behavior of this kernel function in MTEX is to specify the "half-width" (i.e. the full-width at half maximum; FWHM); several choices are shown in Figure 1.9. Now the analogy to equation 1.6 is (Hielscher and Schaeben, 2008):

$$
f(\boldsymbol{g})=\sum_{m=1}^{M} c_{m} \psi\left(\boldsymbol{g g}_{m}^{-\mathbf{1}}\right)
$$

where $\psi$ is the "Ansatz" function (e.g. the de la Vallee Poussin kernel), the $c_{m}$ are integer coefficients, and the $m$ indexes the "nodes" in orientation space where the functions are placed (i.e. the sum is over all nodes $M$ ).

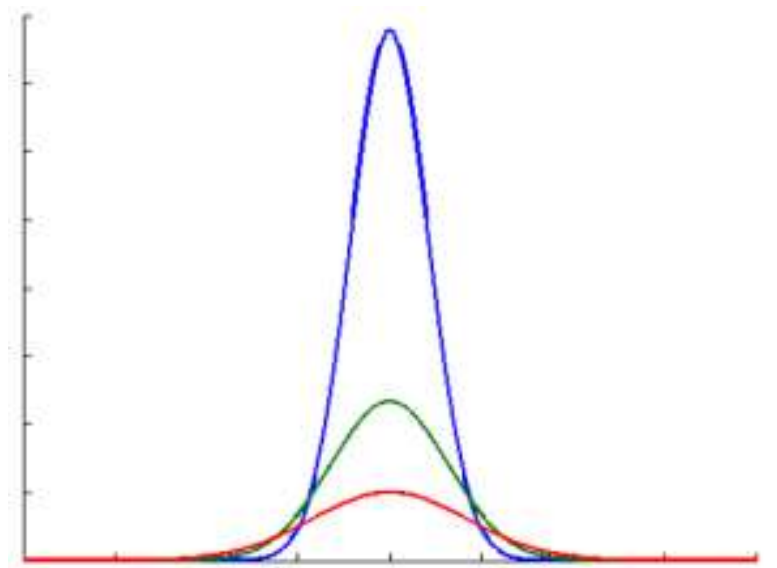

Figure 1.9 De la Vallee Poussin kernel with various half-widths. 
The algorithm is similar to direct methods (e.g. WIMV) because it employs a discretization of orientation (e.g. Euler) space, but in this case the locations of the nodes are determined based on the experimental data, rather than interpolated onto a regular (e.g. $\left.5^{\circ} \times 5^{\circ} \times 5^{\circ}\right)$ grid. This is of course advantageous for analyzing sparse or non-uniform pole figure data. By default, MTEX uses heuristics to determine the "resolution" and half-width based on the experimental data, though it is always an option for the user to specify these quantities instead. Though analyses are often quite sensitive to the choice of kernel half-width, using different resolutions tend to alter the results only slightly, unless the data are indeed very sparse or irregular. This issue will be explored in detail throughout this thesis, most notably in Chapter 2.

Since the algorithm involves fitting a finite series of continuous functions to the experimental pole figure data, it employs least-squares minimization in order to guarantee that the predicted ODF will converge to the experimental data (Van Houtte, 1983). This is performed my minimizing the socalled modified least-squares estimator, which is the squared difference between the re-calculated and experimental pole figures, normalized by the expected variance of the measurement error (Hielscher and Schaeben, 2008). During the computation, the locations of the nodes are determined and radially symmetric, symmetrized (based on input crystal and specimen symmetry) Ansatz functions $\psi$ are placed at each node, but $\psi$ itself (i.e. its form and half-width) is fixed across all nodes. The weighting coefficients $c_{m}$ determine how much weight each node gets and thus the height of the peak centered on the node. After ODF computation, pole figures can be re-calculated in an analogous way to equation 1.8 (summed over the nodes $M$ rather than the cells $J$ ), and the coefficients $C_{l}^{m n}$ (see section 1.4.2.1) can be estimated by a Fourier transform for the purpose of polycrystalline tensor property prediction (Hielscher and Schaeben, 2008).

\subsubsection{Rietveld Texture Analysis (RITA)}

To analyze the large amount of data collected during an energy-dispersive diffraction experiment, techniques have been developed to analyze whole diffraction patterns at once. A common technique originally developed to analyze powder diffraction data is known as the Rietveld method (Rietveld, 1967; Will, 2006), which more recently has been applied to analyze texture (Ischia et al., 
2005; Lutterotti et al., 2007, 1997; Wenk et al., 2010). This is commonly called Rietveld texture analysis (RITA) and one of its principal advantages over traditional methods is that it works even for diffraction patterns with severe peak overlap, such as in low crystal symmetry and/or multi-phase materials. For this reason, it is of special interest in the study of geological materials (Gómez Barreiro et al., 2015; Lutterotti et al., 1997; Wenk et al., 2001). The Materials Analysis Using Diffraction (MAUD) software implements the Rietveld method to analyze diffraction experiments for structure, strain, and/or texture (Kockelmann et al., 2006b; Lutterotti, 2010a; Wenk et al., 2010).

The idea of the Rietveld method is to calculate the intensity at every point in a diffraction spectrum and try to minimize the difference between this model and the experimental data (Lutterotti et al., 2004; Rietveld, 1967; Will, 2006). The refinement itself is performed by sequentially varying chosen parameters in order to minimize the weighted squared sum of differences function:

$$
W_{S S}=\sum_{i}\left[\frac{\left(I_{i}^{\mathrm{obs}}-I_{i}^{\mathrm{calc}}\right)^{2}}{\sqrt{I_{i}^{\mathrm{obs}}}}\right]
$$

where $I_{i}$ is the observed or computed intensity at point $i$ in the diffraction pattern. Though it is typically chosen as the quantity to minimize, $W_{s S}$ is also sometimes used as an indicator of fit quality (Lutterotti, 2010b). Other common indicators are difference plots (i.e. plot of $\sum_{i}\left[I_{i}^{\mathrm{obs}}-I_{i}^{\text {calc }}\right]$ ) and the residual index $R_{w p}$ (Ståhl, 2008):

$$
R_{w p}(\%)=100 \% * \sqrt{\frac{W_{S S}}{\sum_{i}\left[\frac{\left(I_{i}^{\text {obs }}\right)^{2}}{\sqrt{I_{i}^{\text {obs }}}}\right]}} .
$$

In general, the model must account for variations in the intensities, positions and shapes of diffraction peaks based on a variety of factors related to the sample material, type and energy of source radiation, and experimental geometry. The calculated intensity $I_{i}^{\text {calc }}$ at any point $\theta$ in reciprocal space (related to the inter-planar spacing $d_{h k l}$ by equation 1.1), and $(\chi, \varphi)$ in orientation space, is given by (Lutterotti et al., 2004):

$$
I_{i}(\chi, \varphi, \theta)=B k g_{i}+I_{0} L_{p}\left(2 \theta_{i}\right) A_{i}(\chi, \varphi) \sum_{n=1}^{N_{p}} f_{n} \sum_{h=1}^{N_{h k l}} M_{h, n}\left|F_{h, n}\right|^{2} S_{h, n}\left(2 \theta_{i}-2 \theta_{h, n}\right) P_{h, n}(\chi, \varphi)
$$


where $n$ indexes the phases present and $h$ the diffraction peaks (totals given by $N_{p}$ and $N_{h k l}$, respectively). In addition, $B \mathrm{~kg}$ is the background, $I_{0}$ the incident intensity of radiation (x-rays, neutrons, or electrons), $L_{p}$ the Lorentz polarization factor, $A$ the Debye-Waller (or "temperature") factor, $f$ the phase fraction, $M$ the multiplicity, $F$ the structure factor, $S$ the strain factor, and $P$ the texture factor. The latter six of these all depend on the sample itself, while the others depend on the instrument and measurement conditions.

In MAUD, the texture factor is computed by simultaneously refining the ODF along with the structural and instrumental variables. For the harmonic method, this is achieved by including the harmonic coefficients $C_{l}^{m n}$ as refinable parameters and re-calculating pole figures via equation 1.4 to get $P_{h, n}(\chi, \varphi)$ at every iteration. Direct methods are especially suitable for this technique because they are themselves based on an iterative refinement procedure. The weights assigned to each OD cell are refined at the end of each iteration, after the structural and instrumental variables have been refined and the pattern has been re-computed. Hopefully $P_{h, n}(\chi, \varphi)$ starts to converge, leading to better agreement between equation 1.14 and the experimental diffraction spectra.

Depending on the number of phases, the structural complexity of each phase, the number of factors affecting the analysis (e.g. texture, strain, etc.) and the amount of data, RITA analyses can be quite complex and computationally intensive. Such refinements have the tendency to become unstable, and so, unless the analysis is simple, the refinement procedure is quite difficult to fully automate (from a user's perspective) in software. In fact, it is quite difficult even to outline a routine procedure, since it will vary considerably depending on the aforementioned factors. Finally, as with any optimization problem of reasonable complexity, it is quite common that the solution (i.e. minimum) is not unique, and of course there is no guarantee that it will be plausible or physically meaningful (Will, 2006). For these reasons, it is important to approach the Rietveld method with patience and some background knowledge, and to always use caution when interpreting the results. The method will be discussed in detail and example procedures for refinements will be given in Chapters 3and 5. 


\subsubsection{Analysis of Individual Grains}

As mentioned in section 1.4.1, individual grain orientations can be measured by EBSD or synchrotron XRD. If a large number of grains are measured, the data starts to approach the level of statistical smoothness appropriate for ODF computation. Engler (1999) reported that as few as 100200 orientations are needed to compute an ODF that agrees well with results from X-ray pole figure inversion methods, for sufficiently sharp textures in the cubic crystal system. For weaker textures, the minimum is closer to 1000 , and of course more orientations are required the lower the symmetry of the crystal structure. In MTEX, spatially-resolved EBSD data (i.e. an orientation map) are imported, and the software takes a random sample from the presumably large number of grains appearing in the map. Then, the orientation from these grains serve as input for the typical ODF computation algorithm outlined in section 1.4.2.3, i.e. radially symmetric functions are fitted to the discrete set of orientations. The creation of orientation maps is typically done in post-processing software available with most EBSD systems.

\subsubsection{Practical differences}

Quite apart from the philosophical differences between the two broad types of texture analysis approaches, there are also practical differences resulting from the geometry and the radiation source chosen for the measurement. The types of radiation sources used for texture measurement via diffraction are x-rays, neutrons, and electrons (Engler and Randle, 2010). Owing to their higher penetration depths and typically larger beam size, x-rays and neutrons are usually employed for "bulk" or "macro" texture measurements. In other words, the diffracting volume of grains (gauge volume) is large so that the texture information can be regarded as an average over all the grains in that region of the specimen. Electrons have much smaller depths of penetration than either x-rays or neutrons and can be focused to a nano-scale probe, allowing for efficient diffraction measurements of individual grains using EBSD. Because it is inherently advantageous for small-scale, local measurements, EBSD is regarded as a "microtexture" measurement technique (Engler and Randle, 2010). The main difference between $\mathrm{x}$-rays and neutrons is their penetration depths: $\mathrm{x}$-rays are 
strongly absorbed by solids so that generally diffraction information comes from the first $100 \mu \mathrm{m}$ below the surface, whereas neutron experiments typically employ a gauge volume several $\mathrm{mm}$ in depth for most materials. In this way, XRD can be regarded as a near-surface macrotexture technique, whereas neutron diffraction truly probes the bulk. However, both XRD and EBSD can be employed as bulk measurement techniques by removing successive layers and performing measurements on the remaining material.

In recent years, both of these drawbacks of XRD have been addressed to a certain degree by using extremely bright synchrotron x-ray sources, where micro-scale beam sizes have been used with to measure individual grain orientations or at least perform spatially-resolved measurements (Dimasi and Sarikaya, 2004; Wagermaier et al., 2007), and transmission measurements through relatively thick samples have been conducted (Garces et al., 2015a; Yan et al., 2016). Additionally, Budai et al. (2003) created orientation maps (like are common in EBSD) using X-ray "microdiffraction," to study the layer-by-layer texture, crystal structure and strain of oxide thin films grown on metal substrates. Micro-diffraction or "diffraction microscopy" experiments have even been conducted with polychromatic laboratory sources, though the smallest detectable grain using this approach is about $40 \mu \mathrm{m}$ (McDonald et al., 2015). While it will never match the spatial resolution of EBSD or the penetration depths of neutron diffraction, synchrotron XRD is a favorable choice for a wide variety of advanced texture measurement techniques, especially given these recent developments.

The advantages and disadvantages of the various techniques will be explored in more detail throughout the rest of this thesis, most notably in Chapters 4 and 5 .

\subsection{Goals}

The purpose of this thesis is to explore, evaluate and compare various methods for texture measurement and analysis. Chapter 2 studies in detail the impact of using various measurement and analysis procedures on the quality of results for the pole figure inversion technique with XRD and MTEX. A procedure is outlined for reducing the measurement time while maintaining the reliability of pole figure measurements. Chapter 3 explores the use of synchrotron XRD with RITA to analyze 
the textural evolution of an interesting Mg-based alloy during compression testing. The MAUD analysis procedure is described in detail and the benefits and difficulties of RITA are discussed. Chapter 4 applies both XRD and TOF neutron diffraction to measure and analyze the texture of $\alpha$ uranium foils. The results are compared across the methods and to those appearing in the literature. Chapter 5 demonstrates the capability to measure texture on two neutron diffractometers at the Oak Ridge National Laboratory and compares the results from such measurements to those obtained via XRD. A number of analysis procedures are employed and the different results are rigorously and thoroughly compared. Finally, the thesis is concluded and summarized in chapter 6 . Throughout, the reader (and hopefully future texture analyst) is given procedural outlines for measurement and analysis techniques, and the relevance, benefits and drawbacks of each technique are discussed in the context of past, current and foreseeable applications. 


\section{Pole Figure Inversion using MTEX: Comparison of Various Measurement and Analysis Procedures}

\subsection{Motivation}

Texture measurements using monochromatic source radiation require the ability to rotate the sample about a minimum of three axes in order to cover a reasonable range of orientation space. In the standard Bragg-Brentano reflection geometry (Figure 2.1), the rotations about the axes $\theta, \chi$ and $\varphi$, correspond to variation of the diffraction angle, tilt angle, and in-plane rotation angle, respectively (Kocks et al., 1998). A common measurement procedure using this geometry involves fixing the diffraction angle $2 \theta$ while separately varying $\chi$ and $\varphi$ in discrete angular steps, either counting the diffracted radiation continuously or stopping to count at each step. The sample rotations are executed by computer-controlled motors on a texture goniometer (e.g. Huber orienter, Eulerian cradle), and the diffracted intensity is recorded by a movable point detector, or a fixed 2-D position-sensitive detector (PSD) (Engler and Randle, 2010).

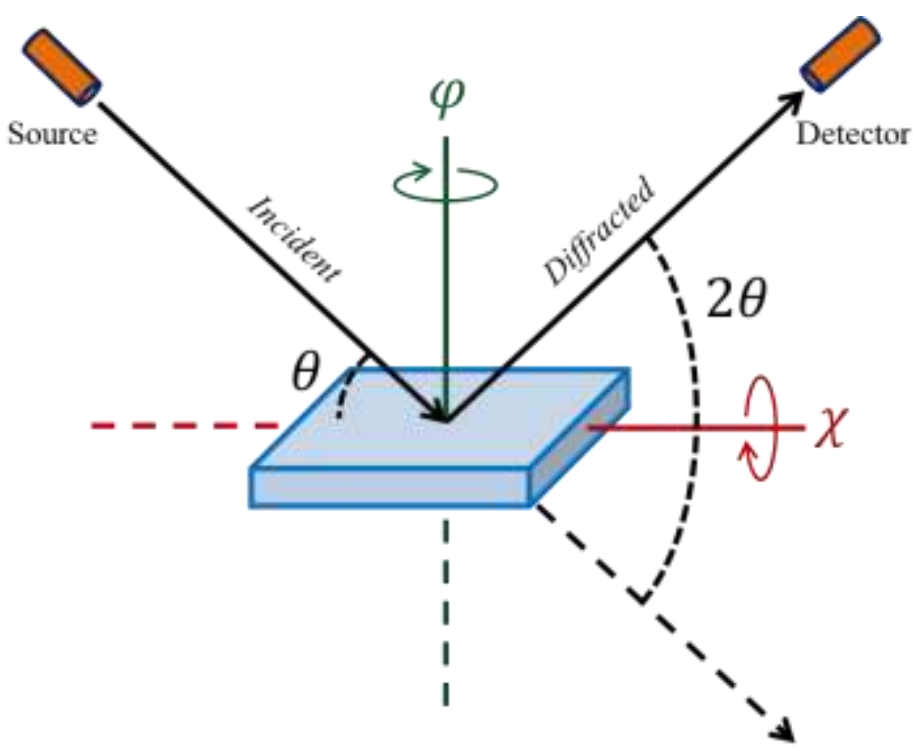

Figure 2.1 Bragg-Brentano reflection geometry, with rotation axes relevant for texture. Note that the sample orientation shown is $(\chi, \varphi)=\left(0^{\circ}, 0^{\circ}\right)$.

It is useful to view the data obtained from this measurement scheme as an intensity function of the two variables $\alpha$ and $\beta$, where $\alpha=90^{\circ}-\chi$, and $\beta=\varphi$. Furthermore, the various sample orientations $(\alpha, \beta)$ can be projected onto a flat circle (via the stereographic projection, for example) 
and represented in polar coordinates, as shown in Figure 2.2. The function $P_{h}(\alpha, \beta)$ is the PDF or pole figure. For a random sample $P_{h}=$ constant for all $\alpha$ and $\beta$, whereas for textured samples $P_{h}=$ $P_{h}(\alpha, \beta)$. With neutron diffraction, it is possible to measure "complete" pole figures, that is covering the full range $0^{\circ} \leq \alpha<90^{\circ}, 0^{\circ} \leq \beta<360$, a considerable advantage over laboratory XRD methods, for which this is not possible due to defocusing beyond about $\alpha=75^{\circ}$ (Engler and Randle, 2010).

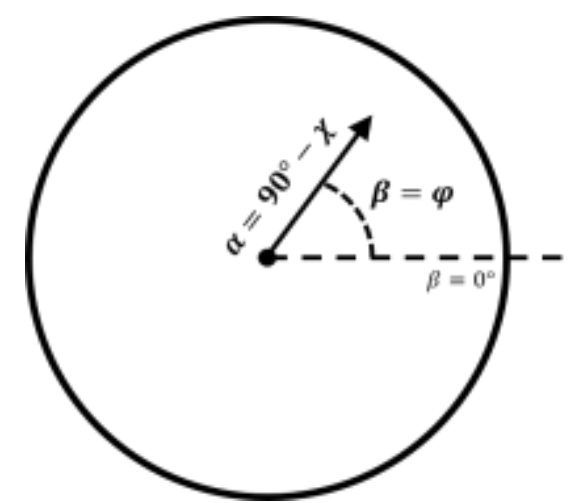

Figure 2.2 Pole figure angles with respect to goniometer rotations.

Successful computation of the ODF generally requires more than one measured pole figure (2-5, depending on the crystal system; see Engler and Randle 2010), each with a large number of measurement points. This, coupled with the longer counting times required for detecting neutrons as compared to x-rays, makes measuring texture with neutron diffraction rather time consuming.

There are two ways to address the issue of long measurement time: maximize intensity in order to minimize the counting time needed per measurement point, or minimize the number of measurement points. Of course, the most efficient method will employ both of these strategies. Intensity can be increased by enlarging the gauge volume, since more diffracting grains contributing to the signal leads to greater intensity. This has the auxiliary benefit of improving grain statistics, but there are limits due to the size of the beam and detector. Minimizing the number of measurement points is slightly more complicated because it involves a balance between efficiency and pole figure resolution/coverage. With too coarse a resolution (i.e. not enough data points), sharp texture components will go unnoticed. The ideal scenario is to reduce the number of measurement points as much as possible, while still producing an accurate result, even for sharp textures. 
The goal of this study is to test, using XRD, a pole figure scanning method which reduces the number of measurement points and thus the measurement time required for texture measurement.

\subsection{Proposed Methodology}

Traditionally, pole figures are scanned on "grids" of equal $\chi$-tilt and $\varphi$-rotation step sizes (usually $5^{\circ} \times 5^{\circ}$ ). This method was designed for laboratory XRD where measurement time is of little concern, and as such it is considerably suboptimal. In particular, viewing a single pole figure in (e.g. stereographic) projection shows that $\alpha$ circles near the inner regions of the pole figure are vastly oversampled, since they contain the same number of data points $\left(360 / 5=72\right.$ for $\left.5^{\circ} \times 5^{\circ}\right)$ over a much smaller arc-length. This oversampling has been shown to yield little information at considerable cost of measurement time, as faster schemes have been proposed and shown to produce nearly identical results (Eichhorn, 1965; Matthies and Wenk, 1992; Rizzie et al., 2008; Tarkowski et al., 2004). However, all of these alternative schemes entirely abandon the equiangular grid and rely on measurements at arbitrary values of $\chi$ and $\varphi$.

In order to use continuous detection, which saves time by eliminating mechanical starts and stops, it is more straightforward from the perspective of data collection and analysis to measure continuously along concentric $\chi$ circles. In other words, the goniometer sits at a given $\chi$ and rotates $\varphi$ a full $360^{\circ}$, while the detector continuously counts. This is repeated for the next $\chi$ and so on, until a full pole figure is collected. After collection, the data can be binned at any values of $\varphi$ along each $\chi$ circle. This is equivalent to the equiangular grid with one difference: the number of measurement points along each $\alpha$ circle is not fixed (72, for $5^{\circ} \times 5^{\circ}$ resolution) but instead depends on the value of $\alpha$, i.e. the radius of the circle. More specifically, moving from outside to inside of the pole figure, the number of measurement points or data bins decreases. This reduces oversampling but still maintains the concentric ring structure, decreasing measurement time with (hopefully) minimal loss of information. The coverage of both schemes is shown in Figure 2.3. 

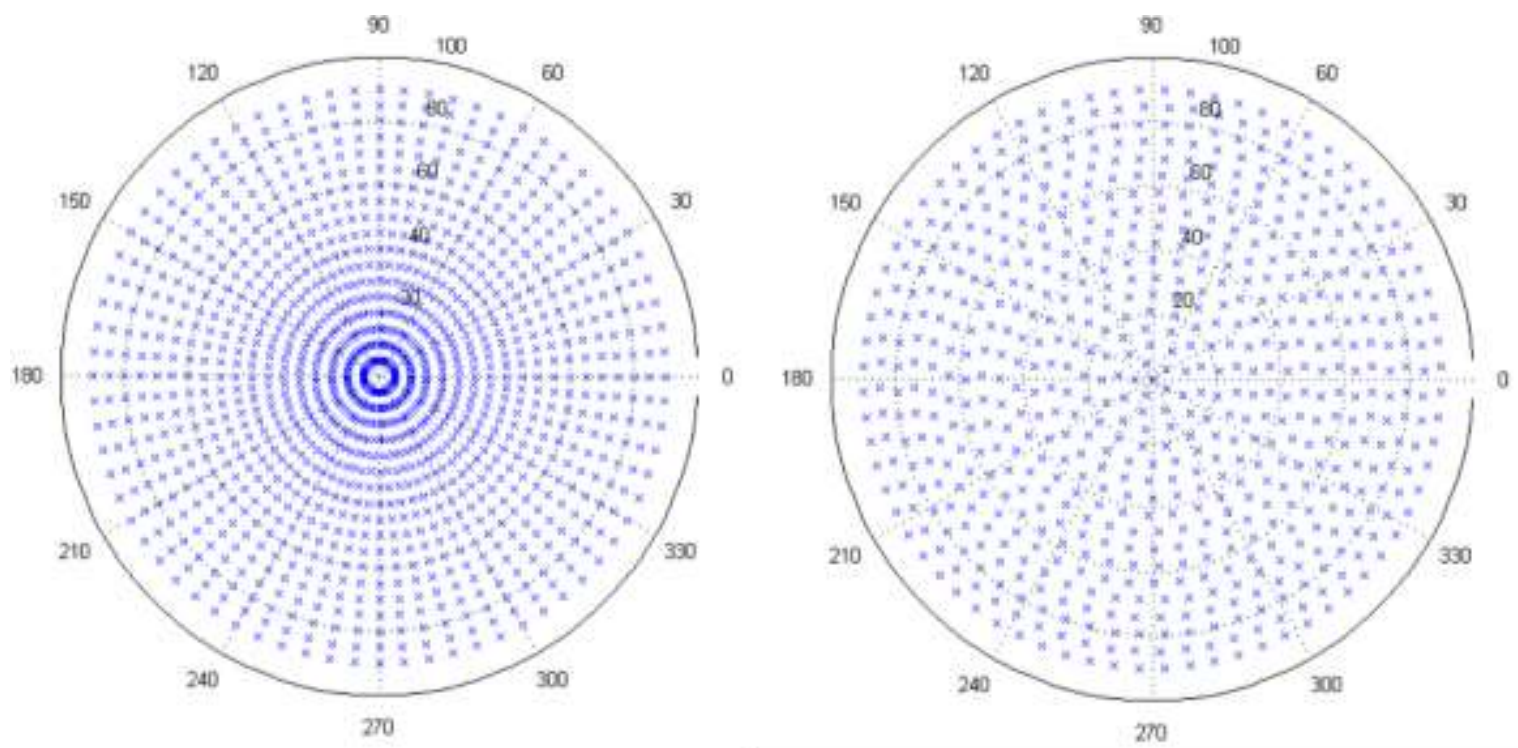

Figure 2.3 Coverage of equal angle (left) vs. proposed grid scheme. The polar angle is $\alpha$ and the azimuthal angle $\beta$.

The idea of the proposed scheme is to closely approximate equal area coverage (as in the hexagonal grid of S. Matthies and Wenk 1992; Rizzie, Watkins, and Payzant 2008) without abandoning the concentric $\alpha$ circle structure. First, the resolution is chosen based on the number of $\beta$ points on the outermost $\alpha$ circle $\left(\chi=0^{\circ}, \alpha=90^{\circ}\right)$ and the number of $\alpha$ circles: for resolution analogous to $5^{\circ} \times 5^{\circ}$, respectively 72 and 18 were used in this study. Then, the arc-length "step" is computed for the outermost circle (the total arc-length divided by the number of $\beta$ points) and the result is used to determine the number of points on all of the remaining circles: the total arc-length of the circle divided by this fixed arc-length step. Since the radius of each circle is simply $\alpha$, the arclength $2 \pi \alpha$ decreases linearly with decreasing $\alpha$. Since it is computed from the arc-length of the first circle, the arc-length step is constant, and so the number of points on each $\alpha$ circle decreases linearly with $\alpha$. Finally, for each circle the starting $\beta$ value is alternated so as to spread the points out and prevent pile-ups, which is not necessary for sufficiently fine resolution but is found to lead to better coverage at coarser resolutions. The savings in number of points approaches $50 \%$ as the resolution gets arbitrarily fine. In this experiment, the proposed grid and the equiangular grid (Figure 2.3) have 684 and 1314 points respectively, a savings of $48 \%$.

\subsection{Experimental Method}


The sample was a small ( $\sim 2 \mathrm{~cm}$ diameter $)$ circular piece cut from a single sheet of basic household-use aluminum foil. The thickness was not measured, but $\mathrm{Al}$ foil is usually about $10-17 \mu \mathrm{m}$ thick ("Thickness of Aluminum Foil," 2013) and at least 99\% pure ("Aluminum Foil: Chemical and Physical Properties," n.d.). The sample was mounted to a noncrystalline glass tray which was attached to the sample stage with double-sided tape.

All measurements were performed on a PANalytical X'Pert Pro MRD, with a Cu X-ray tube source arranged in line focus and operated at $45 \mathrm{kV}, 40 \mathrm{~mA}$. Monochromatic $\mathrm{Cu} \mathrm{K}_{\alpha 1}(\lambda=1.5406 \AA) \mathrm{x}-$ rays were selected from the incident spectrum by mirror optics, and the diffracted intensity was measured with a proportional Xe line detector. The sample stage was an Eulerian cradle capable of rotation about the four axes $\theta, \omega, \varphi$, and $\chi$.

Pole figure scans were performed in step mode (the goniometer stops to measure at each point) both with the standard $5^{\circ} \times 5^{\circ}$ grid and the proposed grid. The first four diffraction peaks in the FCC Al spectrum were chosen for pole figure measurement: (111), (200), (220), and (311), corresponding to $2 \theta=38.41^{\circ}, 44.62^{\circ}, 65.19^{\circ}$, and $78.20^{\circ}$ respectively. For both measurement schemes, a counting time of $1 \mathrm{~s}$ per step was used. Background measurements were attempted, but it was observed that off-peak intensities were so low as to escape detection by the instrument.

Defocusing was ignored because the methods have similar resolutions (and thus will be affected by defocusing in a similar way) near the outer regions of the pole figure, where it might be a significant concern.

\subsection{Results}

Experimental pole figures were plotted with MTEX for both grid methods and are shown in Figure 2.4. The ODFs were calculated for both methods in MTEX using all four experimental pole figures, with default kernel resolution $(R)$ and half-width $(H W)$, as well as $R=5^{\circ}$ and $H W=10^{\circ}$, $15^{\circ}$ (Bachmann et al., 2010). It is possible to query the $R$ and $H W$ that the MTEX heuristic settles on by default; in this case they were $R=5^{\circ}$ and $H W=5^{\circ}$. Pole figures were recalculated from the two ODFs as well as their difference and are shown in Figure 2.5. 


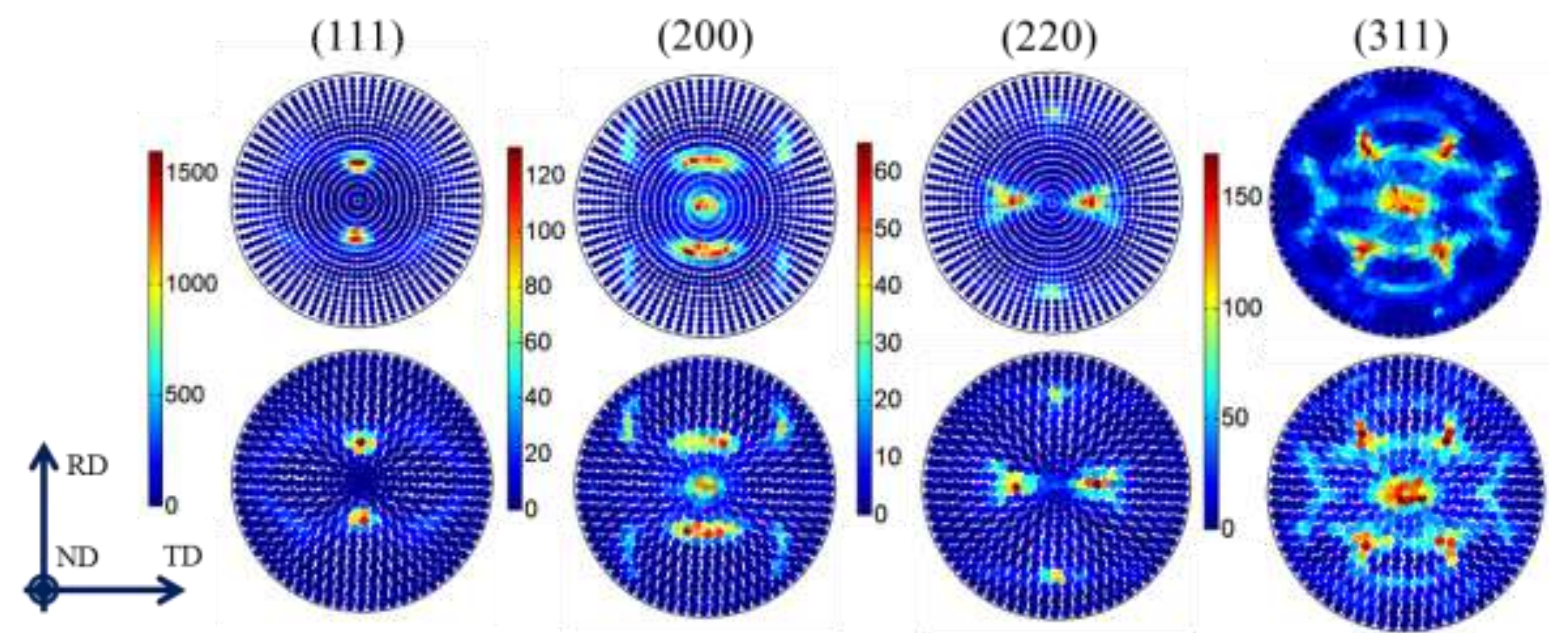

Figure 2.4 Experimental pole figures for $5^{\circ} \times 5^{\circ}$ grid (top) and ring grid. Note that each pole figure is plotted on a different scale, but that the intensity scale is the same across the two methods.

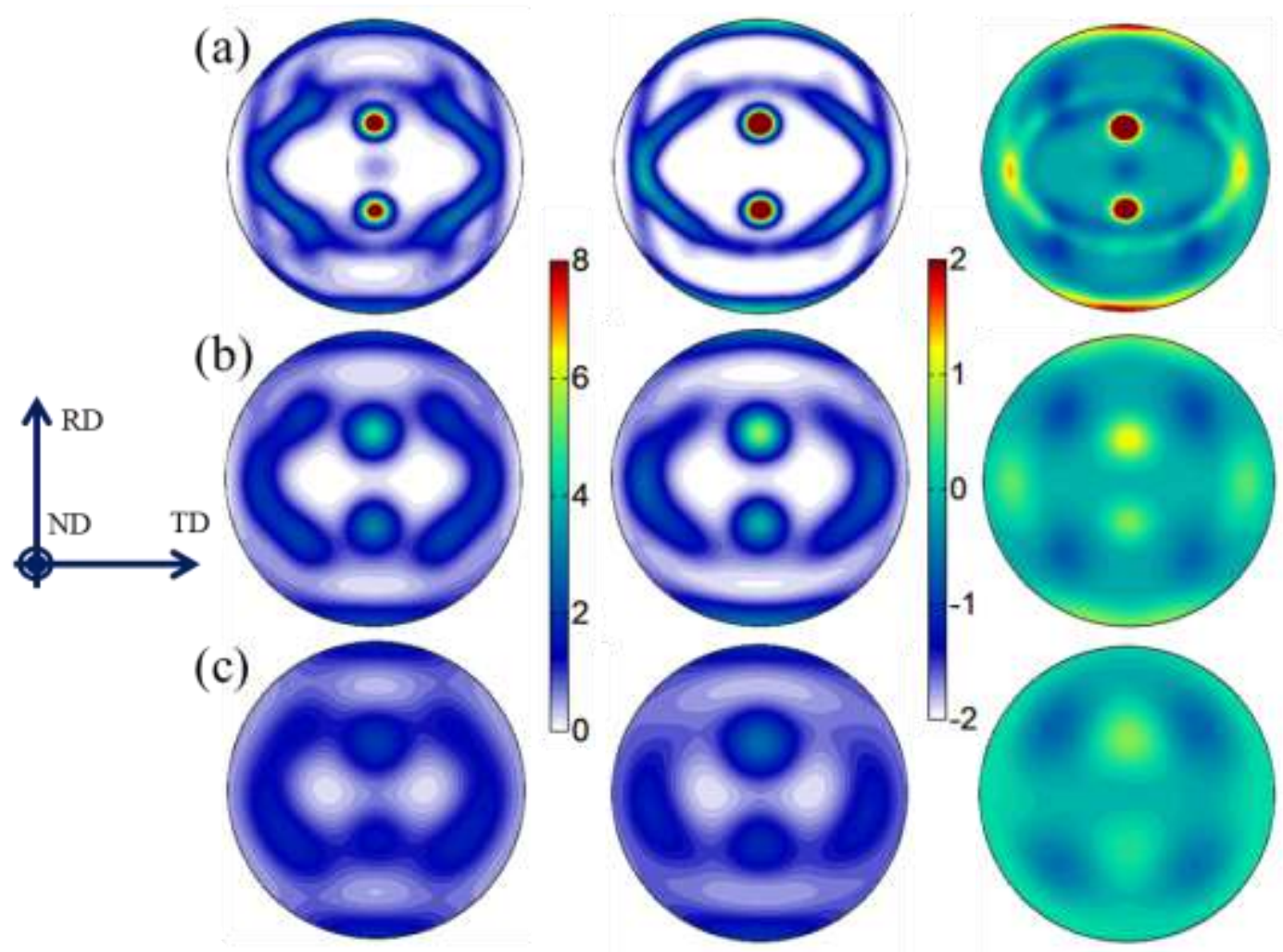

Figure 2.5 $\{111\}$ Pole figures recalculated from the ODF for (from left) $5^{\circ} \times 5^{\circ}$, ring grid and their difference; $R=5^{\circ}$ and $H W=$ (a) $5^{\circ}$ (b) $10^{\circ}$ and (c) $15^{\circ}$.

To more quantitatively examine the differences, the pole figure intensity was plotted as a function of $\beta$ for the $\alpha=35^{\circ}$ ring for both methods. The experimental data are presented in Figure 2.6. In addition, the fits of the ODF computation to the experimental data are displayed in Figure 2.7.

Note that the data are normalized based on the $5^{\circ} \times 5^{\circ}$ and ring grid ODFs (both with default 
resolution and half-width) for plots (a) and (b), respectively. In contrast, the data appearing in Figure 2.8 are normalized based on only the $5^{\circ} \times 5^{\circ} \mathrm{ODF}$ (with default resolution and half-width), in order to compare the fits across the two methods.

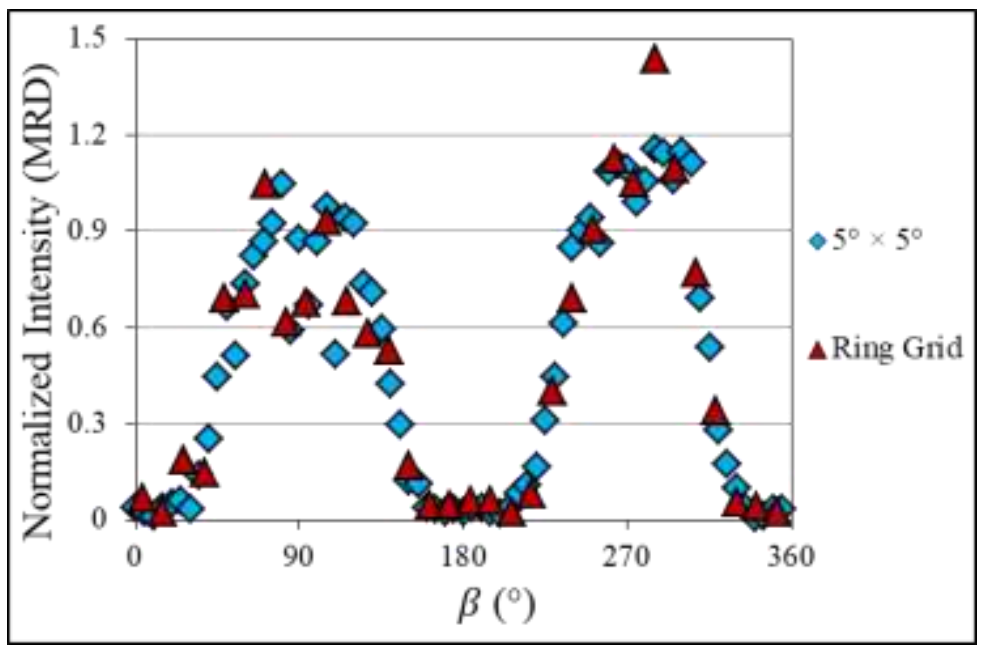

Figure 2.6 Experimental $\alpha=35^{\circ}$ curves for each of the two methods.
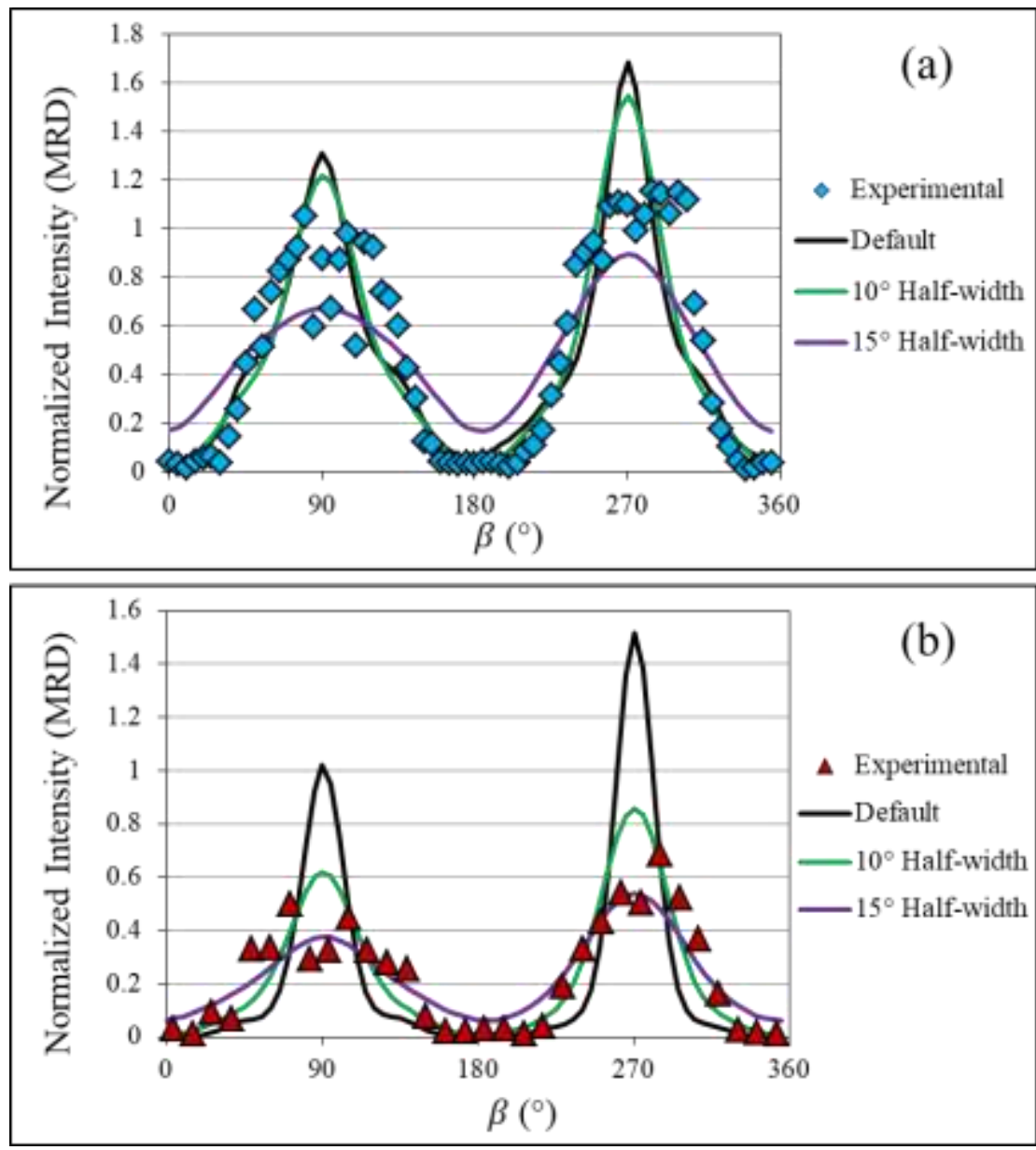

Figure 2.7 ODF fits to $\alpha=35^{\circ}$ curves for (a) $5^{\circ} \times 5^{\circ}$ and (a) ring grid. 

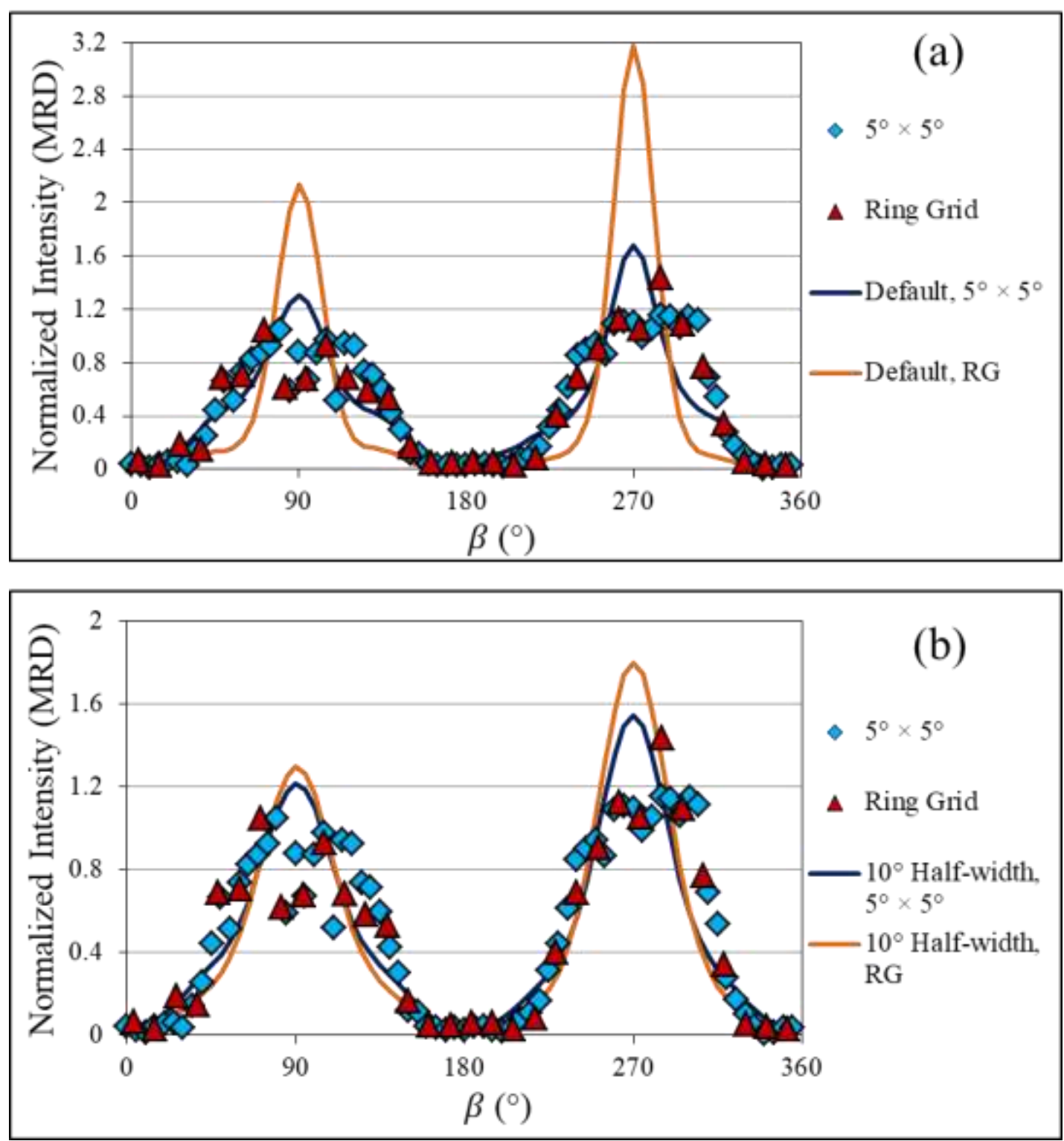

Figure 2.8 Comparison of fits to $\alpha=35^{\circ}$ curves for (a) $R=5^{\circ}, H W=5^{\circ}$ and (b) $R=5^{\circ}, H W=10^{\circ}$.

To more holistically evaluate the texture estimation, orthotropic (point group $\mathrm{mmm}$ or 222) specimen symmetry was imposed and 2-D sections of the ODF were plotted. These are shown for the full range of $\varphi_{2}$ in steps of $5^{\circ}$ for the $5^{\circ} \times 5^{\circ} \mathrm{ODF}$ with $R=5^{\circ}, H W=10^{\circ}$ in Figure 2.9. Then, in Figure 2.10, only the $\varphi_{2}$ sections most appropriate for viewing FCC rolling texture components, which are labeled and consist of the deformation components copper, brass, and S, and the recrystallization components Goss and cube (Engler and Randle, 2010) are shown. The "beta fiber" is also important for FCC rolling textures and is defined approximately by the tube in orientation space running from copper, through $\mathrm{S}$, to brass, but typically one searches for maxima in the neighborhood of the "ideal" components (i.e. copper, $\mathrm{S}$ and brass) along the path $\varphi_{2}=45-90^{\circ}$, rather than just 
reporting the intensity at each component. Figure 2.10 gives a graphical representation of the intensity profile along the beta fiber for the ODFs computed by both methods. Finally, and still under the assumption of orthotropic symmetry, the texture index $J_{O D F}$ and the volume fraction within $\pm 10^{\circ}$ of the components labeled in Figure 2.10(a) were computed and the results compiled in Table 2.1.
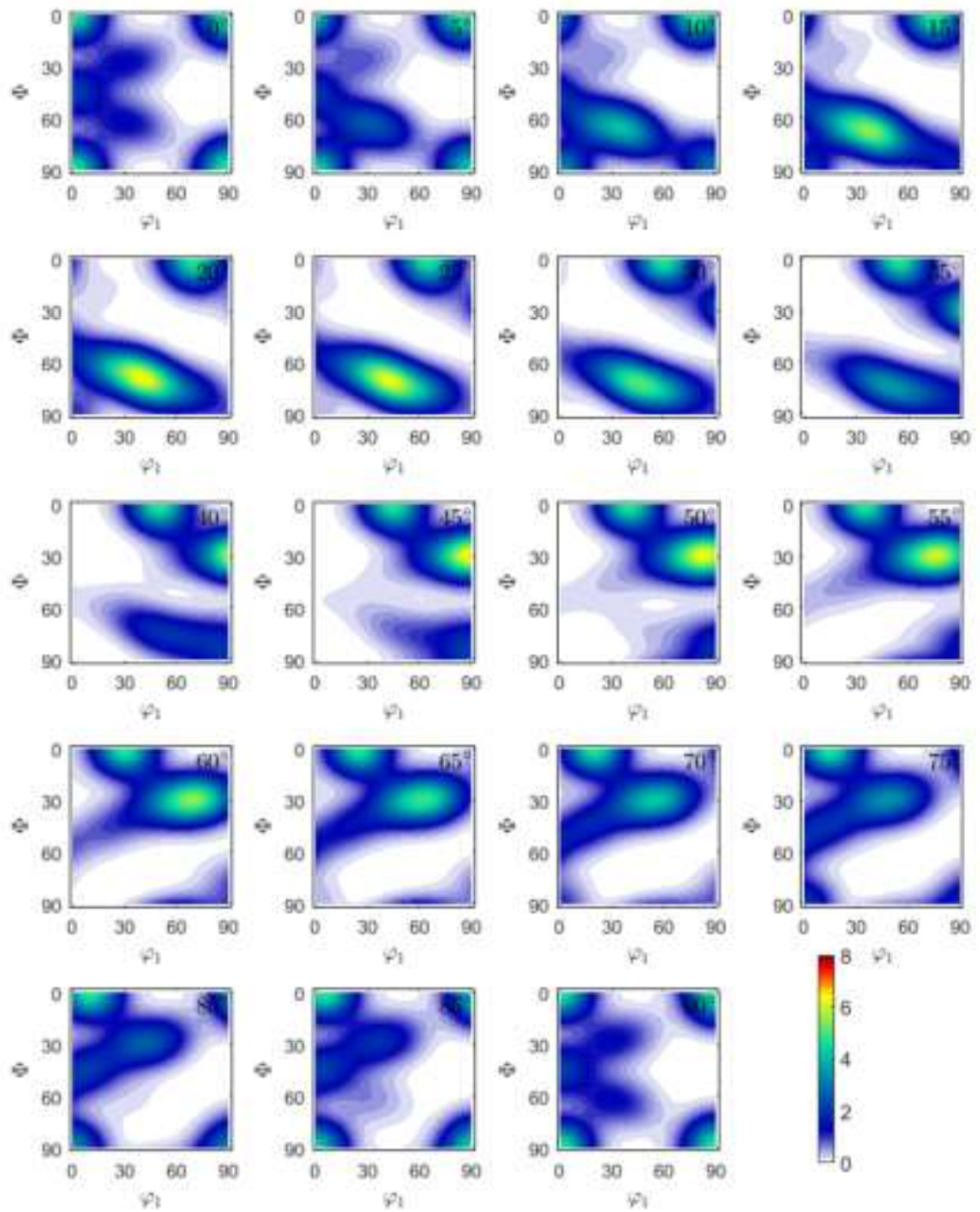

Figure 2.9 Sections $\left(\varphi_{2}=0-90^{\circ}\right.$ in steps of $\left.5^{\circ}\right)$ of the $5^{\circ} \times 5^{\circ}$ grid ODF (orthotropic specimen symmetry) computed using $R=5^{\circ}, H W=10^{\circ}$. 

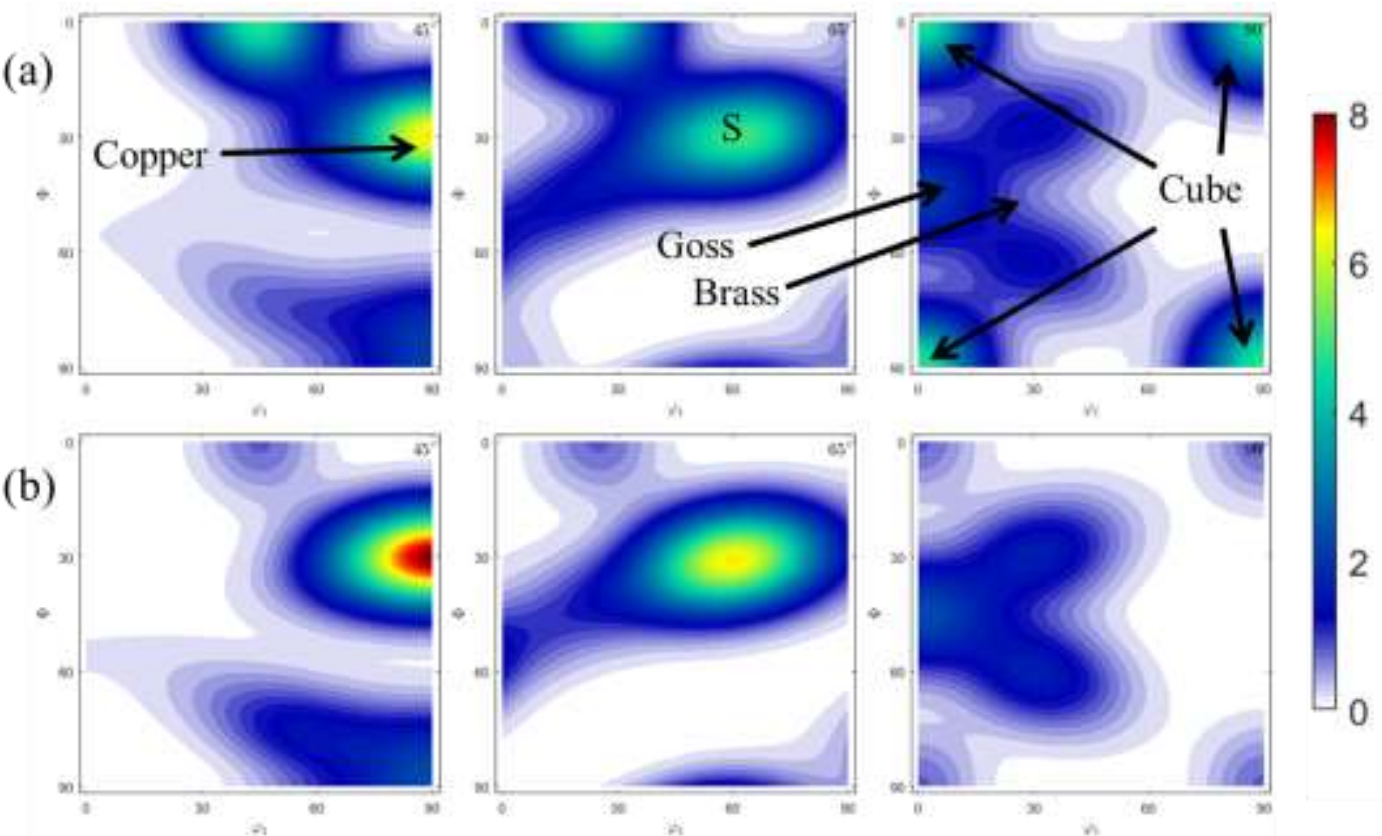

Figure 2.10 Sections $\left(\varphi_{2}=45,65\right.$ and $\left.90^{\circ}\right)$ of the ODF (orthotropic specimen symmetry) computed using $R=5^{\circ}, H W=10^{\circ}$ for (a) $5^{\circ} \times 5^{\circ}$ grid and (b) ring grid. Common FCC rolling and recrystallization texture components are labeled.

Table 2.1 Volume fraction of several FCC rolling and recrystallization components and $J_{O D F}$ for both methods with two $H W$ choices (orthotropic specimen symmetry).

\begin{tabular}{|c|c|c|c|c|c|c|}
\hline & Indices & $\begin{array}{c}\text { Bunge Euler } \\
\text { Angles } \\
\left(\varphi_{1} \Phi \varphi_{2}\right)\end{array}$ & $\begin{array}{c}5^{\circ} \times 5^{\circ} \\
R=5^{\circ}, \\
H W=5^{\circ}\end{array}$ & $\begin{array}{c}\text { Ring Grid } \\
R=5^{\circ}, \\
H W=5^{\circ}\end{array}$ & $\begin{array}{c}5^{\circ} \times 5^{\circ} \\
R=5^{\circ}, \\
H W=10^{\circ}\end{array}$ & $\begin{array}{c}\text { Ring Grid } \\
R=5^{\circ}, \\
H W=10^{\circ}\end{array}$ \\
\hline Copper & $\{112\}<111>$ & $90^{\circ} 30^{\circ} 45^{\circ}$ & 9.3 & 16.2 & 9.7 & 12.7 \\
\hline $\mathrm{S}$ & $\{123\}<634>$ & $59^{\circ} 34^{\circ} 65^{\circ}$ & 9.9 & 13.3 & 8.9 & 11.7 \\
\hline Brass & $\{011\}<211>$ & $35^{\circ} 45^{\circ} 0 / 90^{\circ}$ & 0.5 & 0.4 & 1.8 & 2.6 \\
\hline Cube & $\{001\}<100>$ & $0^{\circ} 0^{\circ} 0 / 90^{\circ}$ & 11.2 & 3.4 & 10.6 & 1.8 \\
\hline Goss & $\{011\}<100>$ & $0^{\circ} 45^{\circ} 0 / 90^{\circ}$ & 6.2 & 8.2 & 5.5 & 5.8 \\
\hline$J_{\text {odf }}$ & - & - & 5.3 & 10.1 & 2.3 & 3.0 \\
\hline
\end{tabular}

\subsection{Discussion}

Though the experimental pole figures for the two methods are quite similar (Figure 2.4), it is clear that the texture strength is dramatically overestimated if the ring grid method is analyzed with default resolution and half-width. The strongest feature appearing in the pole figures is the $\{111\}$ inclined $\sim 35^{\circ}$ from the ND, which is near both the copper and $S$ orientations $\{112\}<111>$, both of which are prominent in cold-rolled pure FCC metals such as Al (Kocks et al., 1998). As shown in Figure 2.4, this feature appears qualitatively similar in both experimental datasets, but Figure 2.7(b) and Figure 2.8(a) show that the MTEX algorithm predicts an overly strong texture if $R=5^{\circ}$ and 
$H W=5^{\circ}$ are used. In fact, the difference between the two ODFs is $89 \%$ at $\beta=270^{\circ}$, despite a difference of only $24 \%$ between the corresponding experimental maxima (Figure 2.6). This, and perhaps similar overestimation of features in other pole figures, causes the significant deviations observable in Figure 2.5(a). Even the $5^{\circ} \times 5^{\circ}$ ODF overestimates the strength near $\beta=270^{\circ}$ by $45 \%$ with default resolution and half-width (Figure 2.7(a)), but this is still a drastic improvement over the $120 \%$ difference in Figure 2.7(b). It is clear that, while neither fit is particularly favorable with $R=5^{\circ}$ and $H W=5^{\circ}$, the use of a finer-resolution grid during the experiment seems to lead to smoother data, which helps the MTEX algorithm find a better fit. For the $5^{\circ} \times 5^{\circ}$ grid, it is evident that even if outliers happen to appear, their overall weight in the ODF computation is less due to the larger number of data points. This is an advantage of sampling heavily near the center of the pole figure.

This analysis of course underscores the importance of using the appropriate half-width and resolution to analyze textures with MTEX, particularly when features differ significantly from the typical bell-shaped kernel form (see section 1.4.2.3; different choices of half-width are also available in MTEX). Of the choices of half-width shown here, $H W=10^{\circ}$ results in the best fit to the experimental data for both collection methods. In general, the best choice will be dependent on the sharpness of the texture and, for unknown textures, should be determined first using analysis of this sort. It should also be noted that various resolutions were tried but the results are often much less sensitive to resolution than to half-width selection, hence the choice to fix the resolution for all ODFs presented here.

In addition to the copper component, the cube and Goss components are quite strong, which is not uncommon for deformed and recrystallized (i.e. hot rolled or cold-rolled and annealed) aluminum (Kocks et al., 1998). The agreement between the two methods in estimating the cube component is extremely poor, which is probably related to the over-estimation of other components (particularly copper) by the ring grid ODF. The cube component is important for balancing the strong rolling texture components that develop during deep-drawing of $\mathrm{Al}$ cans, and under-estimating it so dramatically might result in serious consequences for beverage can production (section 1.1). 
One of the advantages of the proposed method is that its resolution can be increased appreciably while still requiring less measurement points than the traditional $5^{\circ} \times 5^{\circ}$ grid. One could simply increase the number of points on each $\alpha$ circle, the number of $\alpha$ circles, or both, which would still be much more efficient than using a higher resolution equiangular grid (e.g. $\left.2.5^{\circ} \times 2.5^{\circ}\right)$. When use of neutron instrument time is of dire concern, it is worthwhile to first perform a study like this one in XRD. That way, one can know beforehand if the proposed method is suitable for measuring that particular sample.

\subsection{Conclusions}

The method for pole figure measurement proposed in this study produces qualitatively similar results to the standard equiangular method of analogous resolution, but it requires $48 \%$ less data points and therefore results in a considerable reduction in measurement time. For this reason, the method is well suited for neutron diffraction texture measurements, where collection time is a major concern. Since the validity of the method depends on the specific texture, it is additionally recommended that the method first be validated in XRD whenever possible, to ensure that sharp texture features are not being missed or overestimated by the ring grid scheme. This study has also demonstrated the importance of ensuring that pole figure inversion using MTEX is conducted with the appropriate choice of kernel half-width. 


\section{In-situ Texture Analysis of Long-Period Stacking Order Mg Alloys via Synchrotron X-Ray Diffraction}

\subsection{Introduction}

Magnesium alloys are of interest for mechanical and transportation applications because of their exceptionally light weight. However, they often suffer from poor mechanical strength compared to other structural metals like steels or $\mathrm{Al}$ alloys. One method to improve the strength without necessarily sacrificing the ductility is to introduce long-period stacking ordered (LPSO) phases into the $\mathrm{Mg}$ matrix. LPSO phase is known to form in alloys with the composition $\mathrm{Mg}_{97} \mathrm{Zn}_{1} \mathrm{Y}_{2}$, leading to superior mechanical properties (Kawamura et al., 2001). When extruded, the alloy's mechanical properties improve but both the Mg and LPSO phases tend to develop exceedingly sharp textures, which can lead to anisotropy in the deformation behavior (Hagihara et al., 2010a).

Synchrotron X-radiation is ideal for in situ diffraction experiments because of its high brightness and energy (Ángeles et al., n.d.). By equation 1.1 (Bragg’s law), high energy means small diffraction angles (i.e. narrow cones), which allows for direct imaging of full sets of Debye cones with position sensitive detectors. This means that an angular-dispersive measurement can be performed without moving the (source or) detector. High brightness or intensity means that count rates are extremely high, so in-situ studies can be performed with far superior time resolution (especially compared to neutron diffraction). Diffraction spectra can be collected every few seconds or even milliseconds (Van Swygenhoven and Van Petegem, 2013), which is especially useful for studying texture or strain evolution under loading, where strain rates are typically on the order of $10^{-4}-10^{-2} \mathrm{~s}^{-1}$ (Garces et al., 2015a; Liss and Yan, 2010; Zhang et al., 2014).

The purpose of this experiment was to measure the texture and internal strain evolution of an extruded $\mathrm{Mg}_{97} \mathrm{Y}_{2} \mathrm{Zn}_{1}$ alloy with LPSO phase during compression. Owing to the polycrystalline nature of the specimen, $\mathrm{x}$-rays diffract in Debye cones that intersect with the flat detector to form rings. However, a single grain will contribute only a single spot along each ring, and the location of the spots depends on the grain orientation. Thus, the variation in intensity along the ring (i.e. which spots 
are brighter) yields information about the preferred orientation or texture of the specimen, while the spacing between the rings yields information about the lattice spacing and thus the strain in the specimen. The current chapter deals specifically with the analysis of the textural evolution, while authors of a separate manuscript have focused on analyzing the internal strain evolution and the implications of the texture evolution for the crystal plasticity model.

\subsection{Experimental}

\subsubsection{Data Collection}

\subsubsection{Specimen}

A cast billet with nominal composition $\mathrm{Mg}_{97} \mathrm{Y}_{2} \mathrm{Zn}_{1}$ was extruded at $350{ }^{\circ} \mathrm{C}$ and $0.5 \mathrm{~mm} / \mathrm{s}$ in air to a final reduction ratio of 18:1. Cylindrical compression test specimens of $10 \mathrm{~mm}$ length and 5 mm diameter were machined from the extruded bar for diffraction experiments.

\subsubsection{Diffraction Experiment}

In-situ synchrotron x-ray diffraction (XRD) experiments were performed on the P07 HEMS beamline at the PETRA III DESY photon source in Hamburg, Germany (Schell et al., 2008). The high-energy x-ray beam $(\lambda=0.124 \AA$ ) forms a $0.7 \mathrm{~mm}$ x $0.7 \mathrm{~mm}$ spot (defined by the principal slits) on the specimen, leading to a diffracting gauge volume of $0.7 \times 0.7 \times 5=2.45 \mathrm{~mm}^{3}$. The specimen was placed in compression along the axis shown in Figure 3.1, and XRD data were collected at 35 roughly equal strain increments of $\sim 0.4 \%$. The specimen was strained to $9.7 \%$ in total, corresponding to $484 \mathrm{MPa}$ applied stress. 


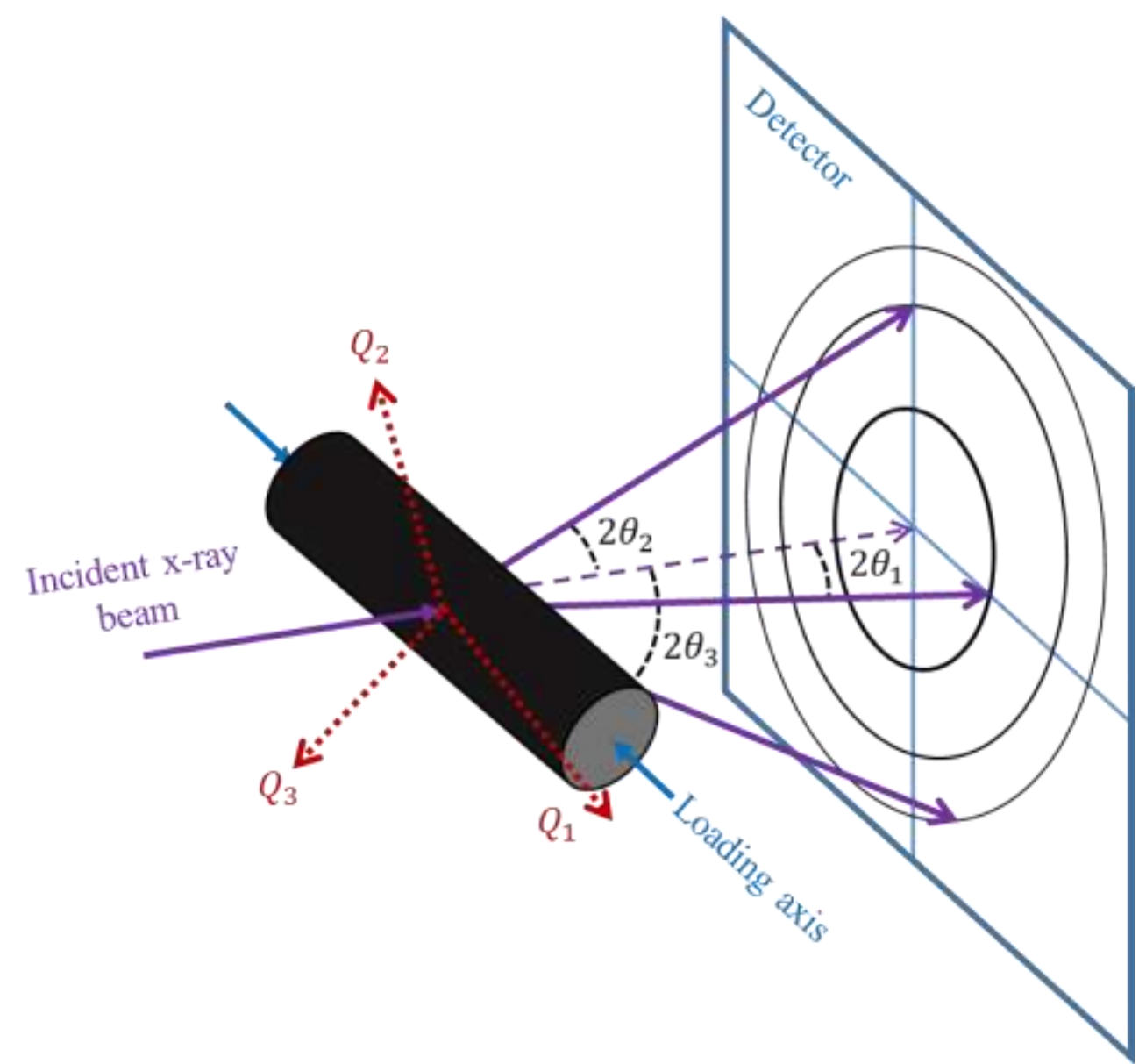

Figure 3.1 Diffraction geometry. Note that $\boldsymbol{Q}_{\mathbf{1}}$ represents diffraction from planes with their poles aligned near the compression axis.

Diffracted X-rays transmit through the sample and are collected on a Perkin Elmer XRD1621 flat panel detector (200 $\mu \mathrm{m}$ pixel size) (Perkin Elmer, 2011), as shown in Figure 3.1. Note that the diffraction vector $\boldsymbol{Q}_{\mathbf{1}}$ is nearly parallel to the compression axis and that the angular deviation from this condition is simply $2 \theta_{1}\left(\sim 2-5^{\circ}\right)$. However, $\boldsymbol{Q}_{2}$ (for example) is exactly a radial direction. Of course, $\boldsymbol{Q}_{3}$ is intermediate to these two.

\subsubsection{Data Analysis}

\subsubsection{Image Processing}

For the texture analysis, the 2-D detector image is converted to a set of diffraction patterns using the ImageJ plugin in MAUD (Lutterotti et al., 1997; Schindelin et al., 2015). The images are corrected for misalignment and rotation and divided into "pie slices" of angular width $5^{\circ}$, corresponding to $360 / 5=72$ diffraction patterns per image. After image processing, RITA was 
performed in MAUD on the set of 72 diffraction patterns, fitting both the $\alpha-\mathrm{Mg}$ (P63/mmc; $\mathrm{a}=3.209$ $\AA$, $c=5.211 \AA$ ) and 18R LPSO (P3212; a = 11.162 ̊, c = 46.899 ̊) phases (Garcés et al., 2014). The refinement procedure is outlined below.

\subsubsection{Rietveld Refinement}

First, 5 overall background parameters* (a $4^{\text {th }}$ degree polynomial) were supplied in addition to one separate background parameter and one scale factor parameter for each of the 72 patterns. There parameters were refined sequentially and then simultaneously. Then, instrument and image processing-dependent parameters, namely x-ray wavelength, sample-detector distance and image centering corrections, were consecutively refined. The volume fractions of each phase were then refined for the initial (zero strain) dataset and fixed for the remainder of the analysis. The angular dependence of peak width was modeled via the Caglioti description (Caglioti et al., 1958), for which the parameters $U, V$ and $W$ were refined (Rietveld, 1969). MAUD also includes a "Gaussianity" term with the Caglioti parameters to model the peak shape as a function of angle; these parameters were refined for each pattern. Each refinement step was performed with 5-10 overall iterations to guarantee convergence (Wenk et al., 2010) with reasonable computation time.

Texture was included as a "variable" in the Rietveld refinement process and, based upon the processing history and prior ex-situ measurements of the texture (Garces et al., 2015a), the assumption of axial (or radial) symmetry was imposed, which is often alternatively described as a "fiber texture" (Kocks et al., 1998). The best fit to the experimental data was achieved using the EWIMV algorithm with $5^{\circ}$ resolution, but other choices were attempted, including standard WIMV with 5 and $10^{\circ}$ resolution, harmonic with $L_{\max }=22$, and E-WIMV with 7.5, 10 and $15^{\circ}$ resolution. In addition, processing the images in slices of $10^{\circ}$ was attempted coupled with $10^{\circ} \mathrm{E}-\mathrm{WIMV}$ and WIMV algorithm choices. All of these alternative choices were found to produce considerably lower quality fits, as gauged by difference plots, $W_{s s}$ values and $R_{w p}$ values. The E-WIMV (or other direct

\footnotetext{
"MAUD allows background parameters to be added one-by-one and the refinement steps repeated. It was determined in this case that 5 parameters yielded a substantially better fit than 4 , but the $6^{\text {th }}$ refined to near-zero. Thus, 5 was chosen to model the background well without unnecessary computational expense.
} 
method e.g. WIMV) computation is implemented in MAUD by adding extra "texture iterations" at the end of each overall refinement iteration (Lutterotti et al., 2004). In this study, the number of texture iterations was fixed at 10 (default in MAUD) during the analysis for a total of $10 n$ iterations where $n$ is the number of overall iterations (varied between 5 and 10 depending on the number of refinable parameters). Strain was accounted for in the analysis by refining "macro-strain" parameters, though this fails to account for the anisotropy in the strain response ("micro-strain"). In other words, as the sample is increasingly deformed, the resulting overall shifts of the diffraction pattern (macro-strain) can be modeled using this approach, but various diffraction peaks shifting independently (microstrain) cannot. Analysis of the micro-strain (or internal strain) was instead considered in the separate study mentioned earlier by tracking the position of individual, well-separated diffraction peaks.

Only a limited number of LPSO peaks are available due to severe overlap at higher diffraction angles, which makes it absolutely necessary to analyze the texture of the LPSO phase with the Rietveld method. Conversely, the behavior of all four $\alpha-\operatorname{Mg}$ peaks $[\{10 \overline{1} 0\},(0002),\{10 \overline{1} 1\},\{10 \overline{1} 2\}]$ in the angular range of interest can be unambiguously described because they are much stronger in intensity than any of the LPSO peaks. For this reason, these reflections were chosen to analyze the internal strain evolution. Only the $\{4 \overline{2} \overline{2} 8\}$ and $\{4 \overline{2} \overline{2} 10\}$ reflections of the LPSO phase were considered in the internal strain analysis.

Both the processing of the 2-D Debye ring images and the full RITA analysis (with the exception of refining the phase volume fraction) were repeated for each of the 35 strain levels, and thus the texture evolution can be described as a function of strain or stress. The texture evolution behavior is important from the perspective of validating the crystal plasticity model (Tahreen et al., 2016) because the type of texture evolution observed can provide clues regarding the deformation mechanisms (dislocation slip and twinning) that accommodate the plastic strain. Each slip system and twinning variant will induce characteristic reorientations of the crystallites during deformation (see e.g. Kocks, Tome, and Wenk 1998). In addition, the initial texture is important because it can serve as an input for crystal plasticity models of the micro-scale response. 


\subsection{Results}

The solution to the Rietveld refinement is shown as a difference plot in Figure 3.2(a) and as fits to the axial and radial experimental diffraction patterns in Figure 3.2(b) and (c), respectively. The $R_{w p}$ value (see equation 1.13 ) was $9.08 \%$ for the initial texture but increased as a function of strain, as shown in Figure 3.3. Pole figures recalculated from the E-WIMV orientation distribution (OD) were exported to MTEX (Hielscher and Schaeben, 2008) and plotted without modification. These are shown for the initial texture of both phases in Figure 3.5. The volume fraction of LPSO was approximately $13.9 \%$.

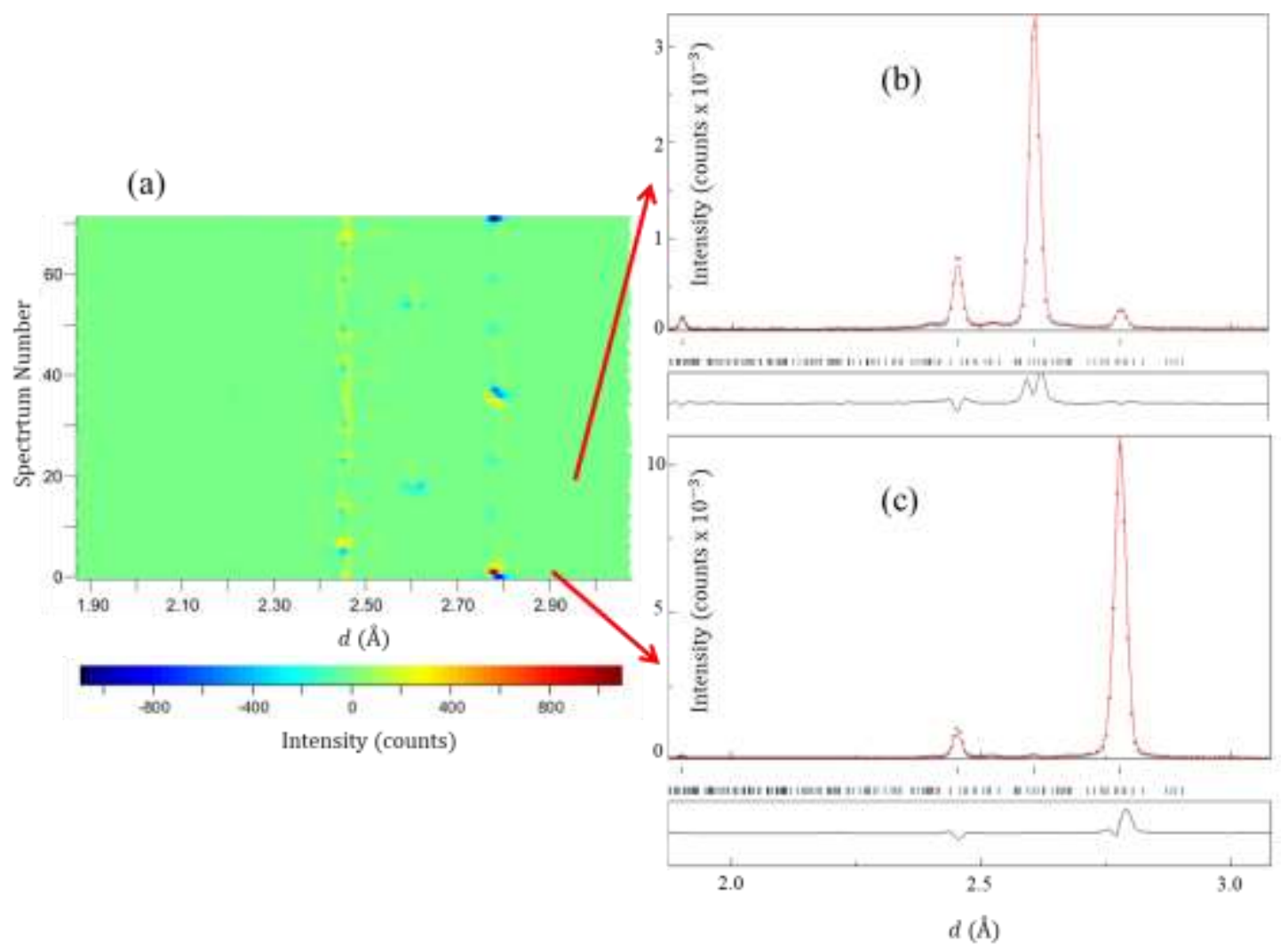

Figure 3.2 (a) Difference plot for all 72 spectra, (b) fit to experimental data for radial pattern, (c) same as (b) for axial pattern. The black dots are experimental data, and the red curve is the Rietveld fit. From top to bottom starting immediately below horizontal axis: $\alpha-\mathrm{Mg}$ peak ticks, LPSO peak ticks, difference curve. 


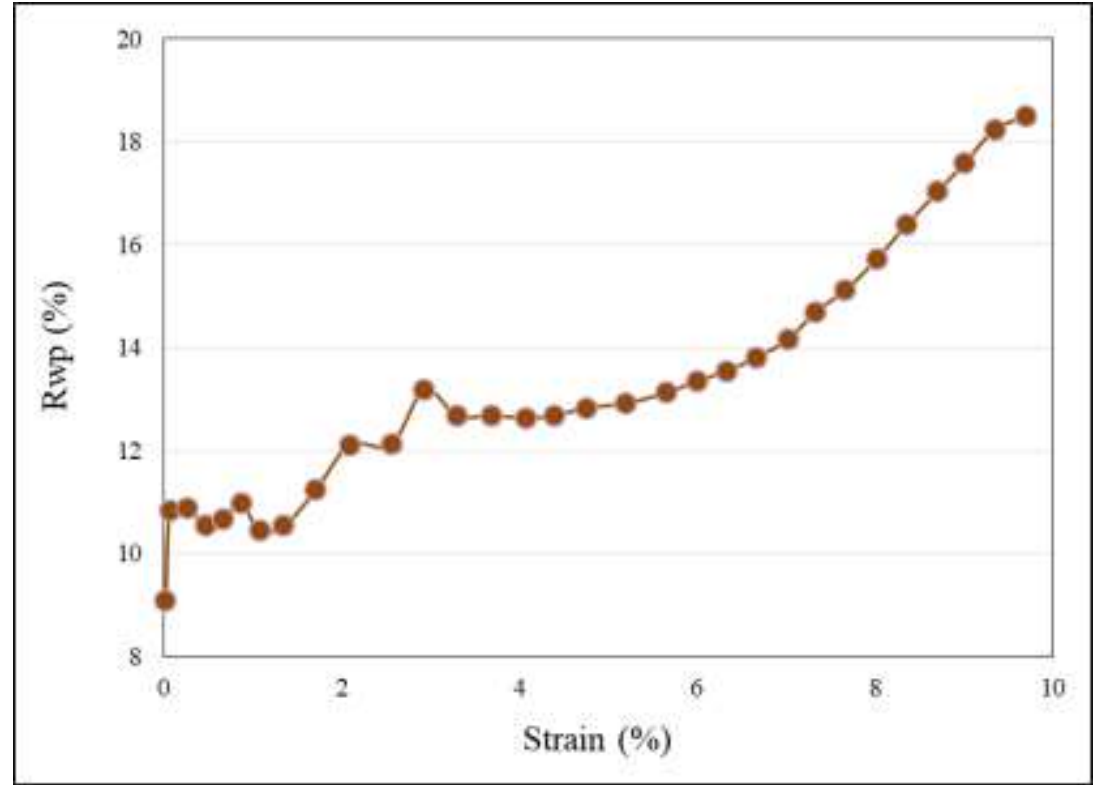

Figure 3.3 Agreement to experimental data $R_{w p}$ (equation 1.13) vs. strain.
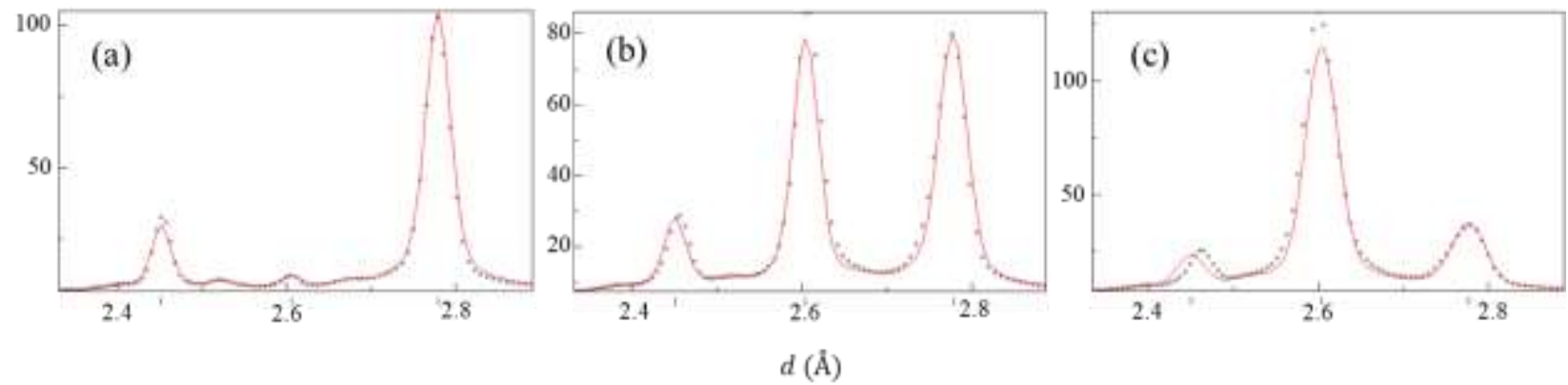

Figure 3.4 Fit to experimental data vs. strain: (a) initial pattern (no strain), (b) intermediate strain ( 6\%) and (c) final pattern $(9.7 \%)$. 


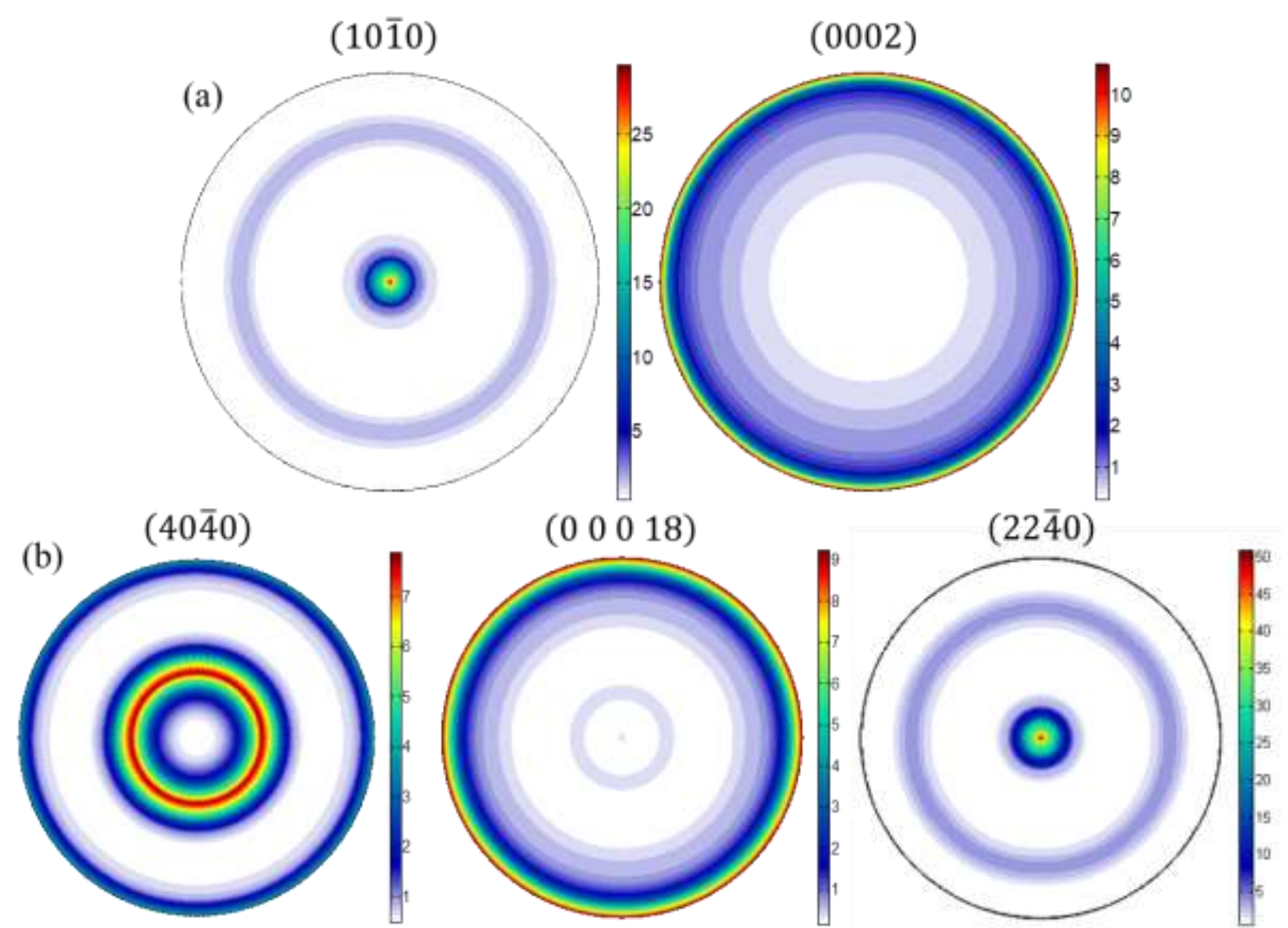

Figure 3.5 Initial texture of (a) $\alpha-M g$ phase, and (b) LPSO phase. The ED (compression axis) is in the center of the pole figure.

The texture evolution can be represented concisely by plotting the axial and radial pole figure intensities as a function of stress or strain. This is done for both phases in Figure 3.6. The axial direction is parallel to both the ED and the axis of compression (orthogonal to the radial direction), and so the axial data are simply the MRD values recorded at the exact center of the pole figure (e.g. in Figure 3.5(a)) while the radial data are from the periphery. The normalization procedure, though consistent across all levels of strain, was different in MAUD vs. MTEX. This is the reason for the quantitative differences between Figure 3.5 and Figure 3.6. Note that any point on the periphery is equivalent due to the fiber symmetry assumption and that logarithmic axes are employed for the intensity, since this permits consideration of the very strong and very weak peaks simultaneously. 
(a)
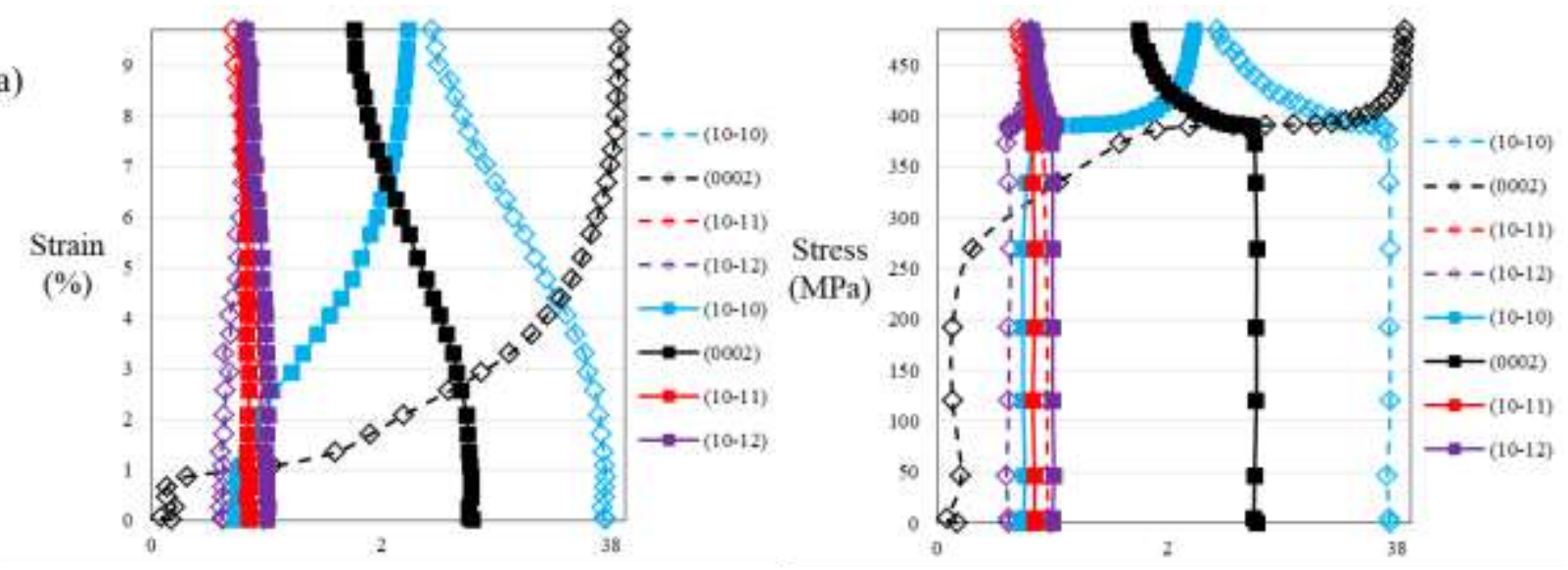

(b)

b)
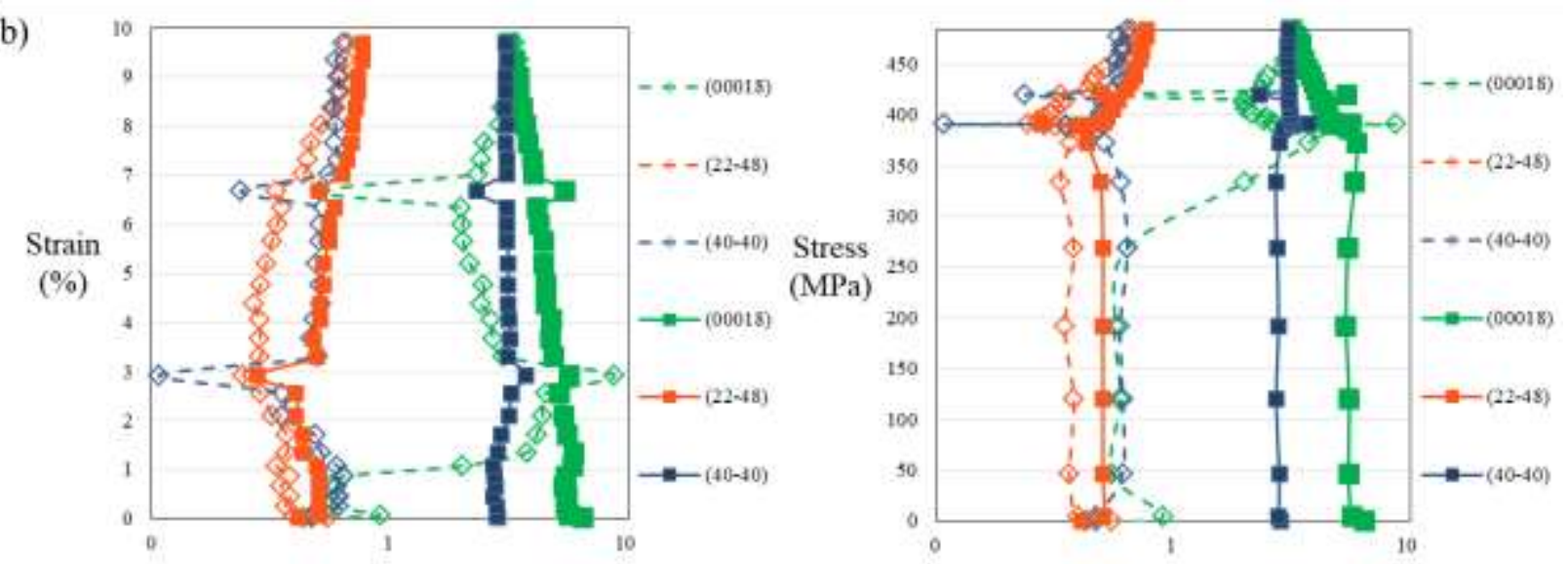

Normalized Pole Figure Intensity $(\log [\operatorname{mrd}])$

Figure 3.6 Evolution of texture as a function of strain and stress for (a) $\alpha-\mathrm{Mg}$ and (b) LPSO. Open symbols and dashed lines are axial (center of pole figure), closed symbols and solid lines are radial (periphery).

Finally, the intensity of the (0002) pole figure was plotted as a function of polar angle for both the initial and final patterns. The results are shown in Figure 3.7. 

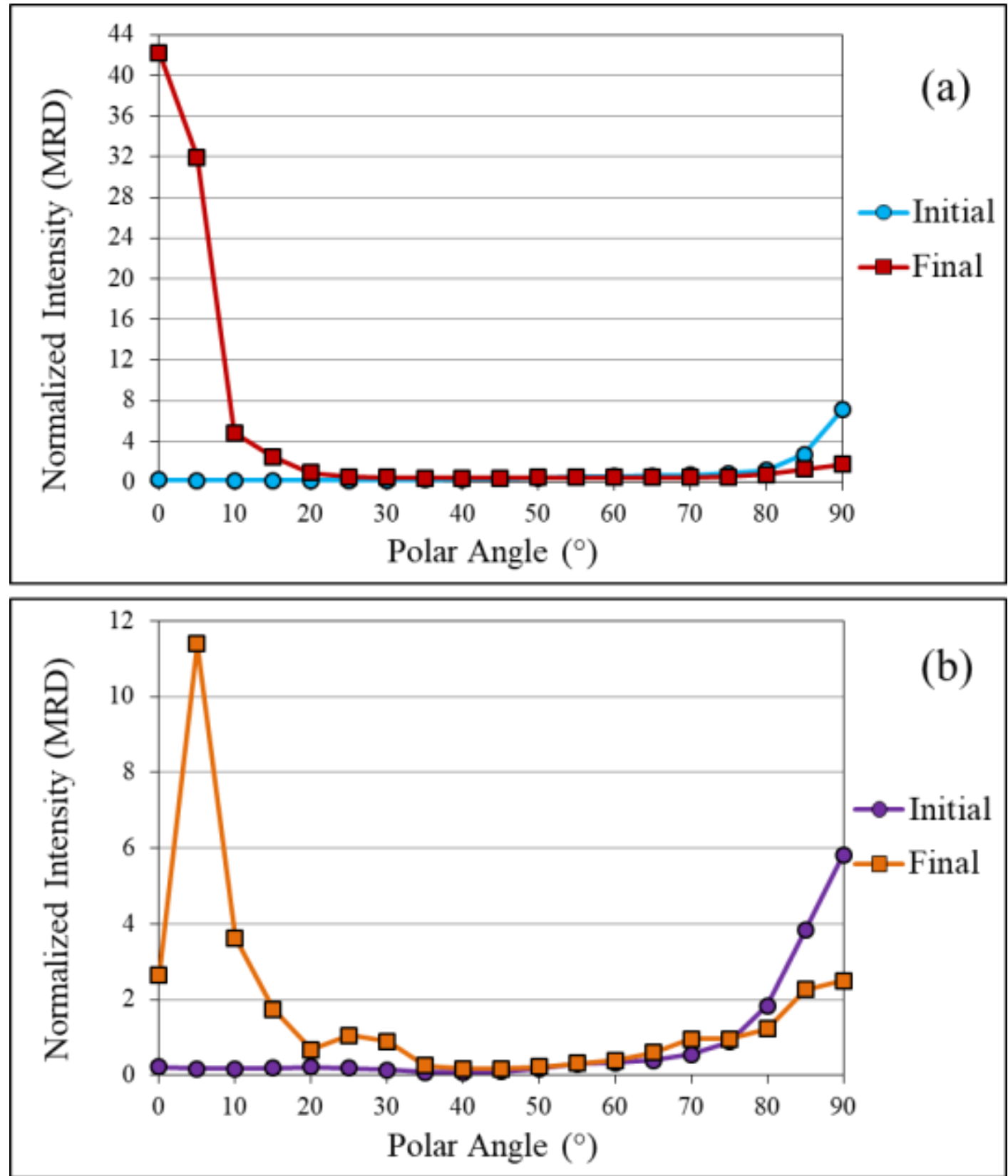

Figure 3.7 Basal reflection intensity vs. polar angle for initial and final texture of (a) Mg and (b) LPSO phases.

\subsection{Discussion}

\subsubsection{Fit to Experimental Data}

The solution to the Rietveld refinement fits the experimental data quite well at zero strain, though it significantly underestimates the axial intensity of the $\{10 \overline{1} 0\}$ reflection $(d \sim 2.778 \AA$; Figure 3.2(a) and (c)). The agreement, as measured by $R_{w p}$ (Figure 3.3), suffers as a function of increasing strain. This is mostly attributable to dramatic and irregular peak shifts that result from elasto-plastic 
anisotropy of the crystals, rather than differences in intensity due to texture. This anisotropy at the grain level results in intergranular stresses that increase with straining, causing the lattice spacings, and thus the diffraction peak locations, to evolve in a way that is difficult to model with full pattern analysis methods. This phenomenon is demonstrated graphically in Figure 3.4 (note especially the $\{10 \overline{1} 1\}$ reflection at $d \sim 2.5)$. This underscores the importance of relying not only on "r-indices"

(including $R_{w p}$ ) but also difference plots to assess the quality of the fit. Such indices only give information on deviation across the whole pattern, whereas difference plots can help determine the specific origin of the deviation (i.e. whether they are due to mismatches in peak position, shape or intensity).

\subsubsection{Initial Texture}

The strong prismatic fiber texture ( $\{10 \overline{1} 0\}$ || ED) present in the $\alpha$-Mg phase initially (at zero strain) is shown in Figure 3.5 and Figure 3.6. This is a typical extrusion texture for this alloy, but the strength of the fiber (maximum $\sim 35 \mathrm{MRD}$ ) is much stronger than previously reported ( 6.5 MRD) on specimens from the same bar in Garces et al. (2015). However, the strength of the texture reported in the previous study may have been significantly underestimated due to the use of the harmonic method to analyze an exceedingly sharp texture (see sections 1.4.2.1 and 1.4.2.2). The texture was also analyzed via pole figure inversion, which relies on well-separated peaks and thus fails to deconvolute the contributions of the LPSO phase to the diffraction pattern. It is also curious that the texture of the same extruded alloy (same extrusion conditions but different specimens) was measured via neutron diffraction and found to have an intermediate value of $\sim 20$ MRD (Garces et al., 2015b). It is possible (but not likely), then, that the difference could be partly related to differences in sampled gauge volume, an issue that will be explored further in chapter 5 .

Results for the same alloy but with different extrusion conditions (i.e. temperature, rate and reduction ratio) have also been reported in the literature. Hagihara et al. (2010) measured the texture via laboratory $\mathrm{XRD}$ of $\mathrm{Mg}_{97} \mathrm{Y}_{2} \mathrm{Zn}_{1}$ bars extruded at $450{ }^{\circ} \mathrm{C}$ and $2.5 \mathrm{~mm} / \mathrm{s}$ in air to a reduction of 10:1. They reported a similarly sharp $\{10 \overline{1} 0\}$ || ED fiber texture with 18.2 MRD maximum intensity and 
suggested that the sharp texture originates from alignment of LPSO grains during extrusion but may weaken during recrystallization. Because of the lower temperature and slow extrusion rate used in the present study, it is likely that the Mg grains experienced little recrystallization and thus little weakening of the initial deformation texture. This is based on the assumption that lower extrusion temperature and rate does in fact result in significantly less dynamically recrystallized (DRXed) grains, which was found to be the case in Hirano et al. (2010).

To investigate the cause of the differences further, a number of approaches to re-analyzing the data were applied. Firstly, the analysis of the initial texture was repeated using the harmonic method $\left(L_{\max }=22\right)$ in MAUD, and the $\{10 \overline{1} 0\} \|$ ED fiber had strength near 20 MRD. This suggests that the differences between the results reported here and those appearing previously in the literature are purely due to differences in analysis procedures (in particular, choice of OD computation algorithm), rather than any experimental anomalies (including gauge volume), since this result is the same as reported in Garces, Perez, et al. (2015) for measurements using neutron diffraction. In addition, the impact of truncation error (see section 1.4.2.1) was assessed by attempting the analysis with $L_{\max }=$ 12 and 8 , and the maximum intensities in the $\{10 \overline{1} 0\}$ pole figures were 11 and $6 \mathrm{MRD}$, respectively. It should also be noted that analyzing the data with direct methods (i.e. E-WIMV) using a resolution coarser than $5^{\circ}$ always resulted in weaker textures. Clearly, the estimated texture strength is sensitive not only to the choice of algorithm type but also to the resolution (in direct methods) or the series expansion limit (in series-based methods).

An additional alternative strategy of importing the MAUD $O D$ (rather than re-calculated pole figures) into MTEX was employed. MTEX requires that OD data imported directly be smoothed by the user's choice of kernel (see section 1.4.2.3; in this case, the default de la Vallee Poussin kernel was selected) before plotting. It was observed that the intensity of the $\{10 \overline{1} 0\}$ || ED fiber was extremely sensitive to kernel half-width and, to a lesser extent, OD resolution. In particular, with a resolution of $5^{\circ}$ and half-widths of $5^{\circ}, 2.5^{\circ}$ and $1^{\circ}$, the maximum intensities of the $\{10 \overline{1} 0\} \|$ ED fiber were 6, 10 and $29 \mathrm{MRD}$, respectively. Again, this demonstrates that the resulting strength is highly sensitive to analysis parameters. 


\subsubsection{Texture Evolution}

With increasing strain, the fiber shifts to $<0001>\|$ ED (basal texture), with similar strength. This phenomenon has been observed previously and is thought to be caused by $<10 \overline{1} 1>$ $\{10 \overline{1} 2\}$ twinning, which rotates (0002) planes by $86^{\circ}$ with respect to their initial orientation (Garces et al., 2015b). In this case, this re-orientation corresponds to the plane normal vectors (reciprocal lattice vectors $\boldsymbol{G}_{0002}$ ) rotating from perpendicular (radial) to parallel (axial) to the compression axis. As expected, the evolution of the radial intensities is complementary to that of the axial ones: the radial intensity of the basal and prismatic poles decreases and increases, respectively, with increasing strain. The simplest way to analyze this in the context of the deformation is by comparing the quantity

$$
\gamma=\int\left[I_{\text {final }}(\alpha)-I_{\text {initial }}(\alpha)\right] \sin (\alpha) d \alpha,
$$

where $\alpha$ is the polar angle over the range where $\left[I_{\text {final }}(\alpha)-I_{\text {initial }}(\alpha)\right] \geq 0$ for the curves in Figure 3.7. This is more reliable than comparing maxima in intensity because it accounts for the different volumes of orientation space occupied by an interval of (say) $\Delta \alpha=5^{\circ}$ at the center vs. the periphery of the pole figure. As expected, $\gamma$ is exactly equal for the axial and radial portions of the patterns even if these portions are defined as $\alpha<30^{\circ}$ and $\alpha>60^{\circ}$, respectively (i.e. ignoring the entire region $\alpha=30-60^{\circ}$ ). Moreover, the fact that the basal intensity shifted from strongly axial to the same strength (as measured by $\gamma$ ) radial suggests that the deformation was accommodated almost entirely by twinning, rather than dislocation slip. In contrast to the sudden and dramatic $\left(86^{\circ}\right.$ in the case of $<10 \overline{1} 1>\{10 \overline{1} 2\}$ ) rotation of crystallites provided by twinning, slip only rotates grains gradually. Thus, if slip were a major contributor, one would expect to see some intensity towards the center of Figure 3.7 (i.e. for $\alpha \sim 30-60^{\circ}$ ), corresponding to crystallites whose basal poles rotated only slightly away from their initial axial orientation. That this is not the case, i.e. that all of the intensity either remains in the region $\alpha \sim 60-90^{\circ}$ (un-twinned grains) or shifts all the way to $\alpha \sim 0-30^{\circ}$ (twinned), and that there is nothing in between (as quantitatively demonstrated by $\gamma_{\text {radial }}=\gamma_{\text {axial }}$, even when the $\alpha \sim 30-60^{\circ}$ region is ignored entirely) suggests that only dramatic rotation characteristic of twinning has occurred. 
Similarly to the initial $\alpha-\mathrm{Mg}$ texture, the LPSO phase initially has its basal poles aligned radially, away from the compression axis. This basal texture is qualitatively consistent with Garces et al. (2014), in which a different alloy of mostly ( $95 \%)$ LPSO phase was analyzed after extrusion, though again the texture is much stronger here. Interestingly, the $\{10 \overline{1} 0\}$ poles align slightly oblique $\left(\sim 25^{\circ}\right)$ to the axis, whereas in this prior study the $\{10 \overline{1} 0\}$ poles were found to align more closely with the ED. An additional noteworthy feature is the extremely sharp $\{22 \overline{4} 0\}$ || ED texture component. Strong alignment of the $\{11 \overline{2} 0\}$ poles along the growth direction was observed in directionally solidified polycrystalline $\mathrm{Mg}_{88} \mathrm{Zn}_{5} \mathrm{Y}_{7}$ (Hagihara et al., 2010b), and this textural feature, though much stronger in this case, was also observed in Garces et al. (2014). Again, the differences in textural strength are likely due entirely to differences in analysis procedures, as the harmonic method produces much weaker results.

Though the LPSO basal texture does not show quite as dramatic a shift as that of the $\mathrm{Mg}$ phase, it is nonetheless prominent. In this case, however, there is some intensity near $\alpha \sim 30-60^{\circ}$, representing grains that have rotated only slightly. Little has been reported on the textural evolution of LPSO in the literature, since an in situ study of this kind is presently unavailable. However, the smaller change may be attributable to the finding that the LPSO tends to deform elastically even after the matrix has yielded (Garces et al., 2015a). It therefore stands to reason that re-orientation due to twinning or would be more prominent in the matrix than in the LPSO phase, while elastic and plastic deformation will more strongly determine the behavior of LPSO grains. Still, it is curious that a considerable volume fraction of grains seem to have re-oriented near axially, as this would suggest the ability of the LPSO phase to twin under compression. This is at odds with the prevailing wisdom (Garces et al., 2015b) and will need to be investigated further by more specifically focusing on the evolution of the LPSO phase in future in situ loading studies.

\subsection{Conclusions}

The texture of an extruded Mg-based alloy with LPSO phase was analyzed via in-situ synchrotron XRD. An exceedingly sharp $\{10 \overline{1} 0\}$ ॥ ED fiber texture, which can perhaps be explained 
by the low extrusion temperature and rate, was observed initially. Some previous reports suggested that the texture was considerably weaker, but the following problems with those measurement and analysis procedures provide us with confidence in the present assertion that the texture is indeed very strong. All of the prior results were based on pole figure measurements via neutron diffraction or laboratory XRD and were analyzed with the harmonic method, which may cause considerable underestimation of the texture strength. A direct method such as E-WIMV is a more suitable choice for analyzing extrusion and other sharp textures, as series-based (e.g. harmonic or MTEX) methods tend to artificially smooth sharp features. In general, full pattern analysis (i.e. RITA) methods will yield more reliable results with the added benefit of being able to analyze the texture of the LPSO phase with relative ease. This is not the case for pole figure inversion methods where severe peak overlap makes it difficult to resolve the intensity contributions of individual poles to diffraction peaks. The texture of the LPSO phase was also analyzed and, again, was found to be qualitatively similar to but much stronger than results in the literature from pole figure inversion methods.

As the sample was compressed, the fiber shifted towards $<0001>\|$ ED with similar strength. This is understood to occur because of twinning, and the results presented here suggest that the deformation must have been accommodated in the matrix almost entirely by $<10 \overline{1} 1>\{10 \overline{1} 2\}$ twinning. The LPSO texture also shifted during compression, though the overall change was smaller than for the Mg texture, suggesting that the plastic deformation is accommodated less by twinning and more by dislocation slip in the LPSO phase. A study dedicated to tracking the evolution of the LPSO phase under load should be conducted to aid in understanding of the deformation behavior of this phase. 


\section{Crystallographic Texture of Straight-Rolled $\alpha$ - Uranium Foils via Neutron and X-ray Diffraction}

\section{Attribution:}

The following text is taken from a publication appearing in the June 2017 issue of The Journal of Applied Crystallography, vol. 50 (3), p. 859-865. With the exception of some minor formatting changes (for consistency with the rest of the thesis), it appears exactly as published. X-ray texture measurements and analysis using MTEX were performed by John Einhorn at the University of Virginia. Neutron diffraction measurements were performed and analyzed preliminarily by Dr. Sven Vogel at the Los Alamos National Laboratory. They were further analyzed with MAUD by John Einhorn. Much of the literature review was performed by Dr. Matt Steiner, and the manuscript was written jointly by Dr. Steiner and John Einhorn. All authors contributed to editing both before and after initial submission. Dr. Steiner handled submission and all correspondence with reviewers. Additional acknowledgements concerning sample preparation and funding of the work are given at the end of the chapter. 


\title{
Crystallographic Texture of Straight-Rolled $\alpha$-Uranium Foils via Neutron and X-ray Diffraction
}

\author{
J.R. Einhorn', M.A. Steiner ${ }^{*}$, S.C. Vogel', E. Garlea ${ }^{3}$, and S.R. Agnew \\ ${ }^{1}$ University of Virginia, Material Science and Engineering, 395 McCormick Rd, Charlottesville, VA 22904, USA \\ ${ }^{2}$ Los Alamos National Laboratory, Los Alamos, NM 87545, USA \\ ${ }^{3}$ Y-12 National Security Complex, Oak Ridge, TN 37831, USA \\ *Corresponding Author: mas4cw@virginia.edu
}

\subsection{Abstract}

The texture of recrystallized straight-rolled $\alpha$-uranium foils, a component in prospective irradiation target designs for medical isotope production, has been measured by neutron diffraction, as well as X-ray diffraction using both $\mathrm{Cu}$ and Mo sources. Variations in the penetration depth of neutron and X-ray radiation allow for determination of both the bulk and surface textures. The bulk $\alpha$ uranium foil texture is similar to the warm straight-rolled plate texture, with the addition of a notable splitting of the (001) poles along the transverse direction. The surface texture of the foils is similar to the bulk, with an additional (001) texture component that is oriented between the rolling and normal directions. Differences between the surface and bulk textures are expected to arise from shear forces during the rolling process and the influence that distinct strain histories have on subsequent texture evolution during recrystallization.

Keywords: $\alpha$-uranium, rolling texture, neutron diffraction, X-ray diffraction, uranium foil

\subsection{Introduction}

The crystallographic texture of uranium can have a significant impact on its bulk properties and mechanical behavior as the room temperature allotrope, the orthorhombic $\alpha$-phase $(a=2.85 \AA$, b $=5.87 \AA, c=4.94 \AA$ ), has unique anisotropic properties including a negative thermal expansion coefficient along the crystallographic b-axis (Anderson et al., 1982; Burke and Turkalo, 1957; Foote, 1956; Sherby et al., 1976). Even with highly anisotropic single-crystalline properties, polycrystalline materials that are randomly textured can still have largely isotropic bulk properties. As $\alpha$-uranium is known to develop relatively strong textures during processes such as rolling, extrusion, annealing, and even casting (Sturcken and Walter, 1974), polycrystalline anisotropy in $\alpha$-uranium must often be 
taken into account. Owing to anisotropic thermal expansion and the resulting grain-grain interactions, residual stresses and even macroscopic dimensional instabilities can develop from temperature changes in polycrystalline $\alpha$-uranium (Calhoun et al., 2015; Salice and Zein, 1961). Plastic deformation in polycrystalline $\alpha$-uranium is heavily dependent on the presence and relative strength of specific texture components (Cahn, 1953; Zecevic et al., 2016a). Since the thermomechanical properties of $\alpha$-uranium vary with texture, understanding how texture develops during processing is critical to optimizing material performance during service.

A number of recent studies have investigated the texture of $\alpha$-uranium as it undergoes different types of deformation (Brown et al., 2009; Calhoun et al., 2015; Choi and Staker, 1996; Knezevic et al., 2013a, 2013b, 2012; McCabe et al., 2010; Zecevic et al., 2016a), but these typically focus on samples deformed only to moderate strain levels. The present study investigates an extreme case, where a plate has been rolled progressively until reaching a foil thickness of less than $200 \mu \mathrm{m}$. Foils of $\alpha$-uranium are of technical interest as a means of breeding medical radioisotopes such as Technetium-99m (Tc-99m), which is used in approximately $85 \%$ of nuclear diagnostic imaging procedures worldwide (Bradley, 2013). Traditionally, Tc-99m is produced by irradiating dispersion targets of highly enriched uranium (HEU) in an aluminum alloy matrix. The matrix contains the fission products and facilitates cooling, but also contributes unnecessarily to the liquid waste stream as the entire target must be dissolved to remove the fission products (Bradley, 2013; Lokhov, 2010). The ability to physically separate the aluminum before chemical extraction of the radioisotopes would greatly reduce the liquid waste stream and enable the use of targets manufactured with low enriched uranium (LEU). LEU produces a lower fission yield than HEU, but also reduces proliferation risk, has less stringent export controls, and can be irradiated in a larger fraction of the world's nuclear reactors (Bradley, 2013; Lokhov, 2010; Whipple and Larson, 2009). One promising target design strategy involves cladding $\alpha$-uranium foils in a recoil barrier, placing the assembly between two aluminum tubes, and then closing the gap between them with hydraulic pressure (Govindarajan et al., 2013; Makarewicz and Solbrekken, 2013; Snelgrove et al., 1995; Turner et al., 2009). This target can be disassembled post-irradiation, so that the uranium foil is processed separately from the tubes, but it requires intimate contact of the foil with the tubes during irradiation in order to transfer heat to a 
surrounding coolant. As a result, it is necessary to determine the crystallographic texture produced during the processing of $\alpha$-uranium foils in order to accurately predict their anisotropic aggregate polycrystalline properties and ensure that no gaps will form.

This study investigates the crystallographic texture of straight-rolled $\alpha$-uranium foils using neutron diffraction, as well as diffraction with both $\mathrm{Cu} K \alpha(\lambda=1.54 \AA)$ and $\mathrm{Mo} \mathrm{K} \alpha(\lambda=0.71 \AA) \mathrm{X}$ ray sources. The diffracting material volume will be dependent on both the wavelength and type of radiation. To contribute to a diffraction spectrum, incident radiation must penetrate and then exit the material, facing attenuation due to absorption and scattering (coherent and incoherent) along each path length. The intensity after attenuation can be calculated using the familiar Beer-Lambert law:

$$
I=I_{0} \exp (-a x)
$$

where $a$ is the radiation's linear attenuation coefficient in the material, $I_{0}$ is the initial intensity, and $x$ is the path length (Hutchings et al., 2005). The attenuation coefficients for X-ray radiation in $\alpha$ uranium are $5458 \mathrm{~cm}^{-1}$ for $\mathrm{Cu} \mathrm{K \alpha}$ and $1933 \mathrm{~cm}^{-1}$ for Mo Ka (Fultz and Howe, 2008; Saloman and Hubbell, 1988), taking the density of $\alpha$-uranium to be $18.95 \mathrm{~g} / \mathrm{cm}^{3}$ (Hutchings et al., 2005). The neutron scattering cross-section will be wavelength dependent unless the source is monochromatic, but at the $1.8 \AA$ wavelength commonly used for comparison, the attenuation coefficient for depleted $\alpha$-uranium is $0.43 \mathrm{~cm}^{-1}$, which is fairly representative over the wavelength spectrum employed in this study (Hutchings et al., 2005; Sears, 1992). Figure 4.1 plots the relative attenuation of the three radiation sources as a function of path length. $\mathrm{Cu} \mathrm{K \alpha} \mathrm{X}$-rays reaching the detector during texture measurements have traveled a mean path length through the foil of $1.37 \mu \mathrm{m}$. Higher-energy Mo K $\alpha$ X-rays are able to travel further through the material, with a mean path length of $4.69 \mu \mathrm{m}$, though the lower diffraction angles corresponding to the higher wavelength (compared to $\mathrm{Cu} \mathrm{K} \alpha \mathrm{X}$-rays) increase the necessary path length for diffraction, partially offsetting the gain in penetration depth. Both X-ray sources are limited to measuring only the first few micrometers at the foil surface. In stark contrast, neutrons are able to easily penetrate the entire thickness of the $\alpha$-uranium foil with only minimal attenuation due to their relatively weak interaction with matter (Engler and Randle, 2010). This disparity in the penetration depths allows for the foil texture at the surface and over the full thickness of the foil to be determined independently and compared. 


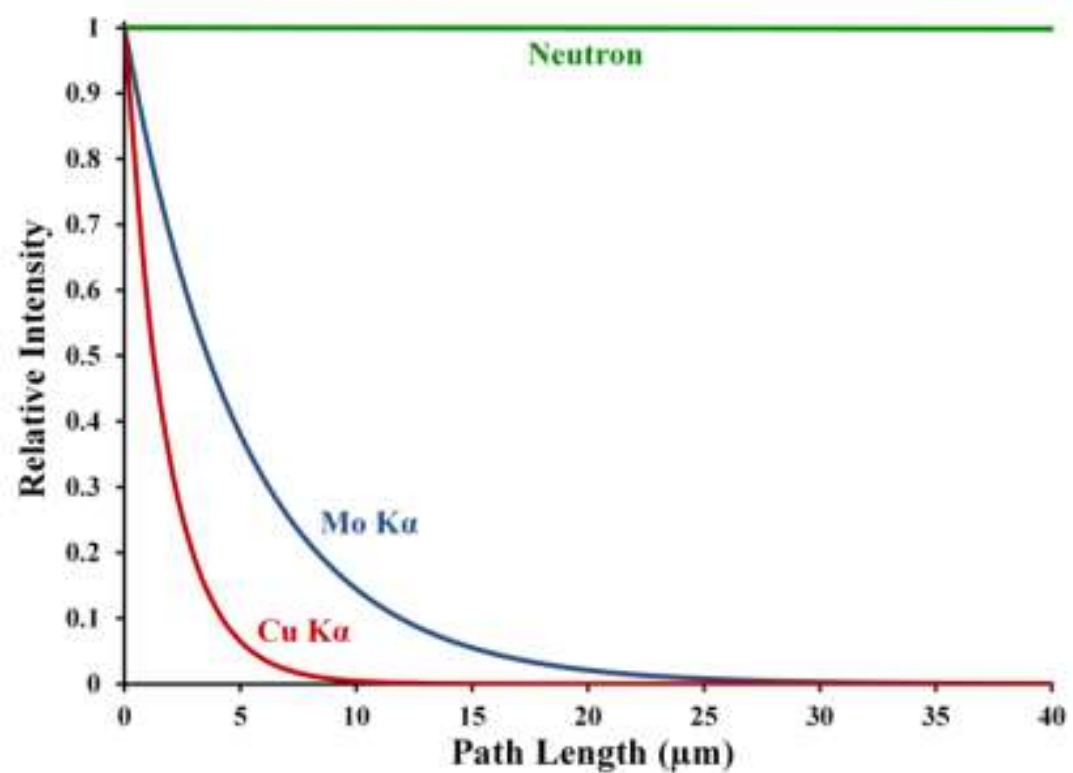

Figure 4.1 Attenuation relative to the initial intensity of $\mathrm{Cu} K \alpha$, Mo $\mathrm{K} \alpha$ and neutron radiation in depleted $\alpha$ uranium, as a function of path length through the material. The path length required for diffraction is considerably longer than the depth into the surface

\subsection{Experimental Methods}

The foils analyzed in this paper started as an approximately $6 \times 10 \times 1.25 \mathrm{~cm}$ depleted uranium $\left(0.3 \% \mathrm{U}_{235}\right)$ casting in a graphite mold. The casting was heated to $630^{\circ} \mathrm{C}$ (below the $\alpha \rightarrow \beta$ phase transition at $661{ }^{\circ} \mathrm{C}$; see Rechtien and Nelson 1973) in a molten salt bath and hot, cross rolled twice, with each pass reducing the thickness approximately $10 \%$, introducing a cumulative hot reduction of about $34 \%$. The hot rolled plate was then cold straight-rolled in forward and backward passes until reaching a thickness of $300 \mu \mathrm{m}$, a thickness reduction of around $95 \%$ from the hot rolled plate. Oil was used as a lubricant during cold rolling. At a thickness of $300 \mu \mathrm{m}$ the foil had work hardened significantly and was subsequently annealed at $630^{\circ} \mathrm{C}$ for 2 hours to restore ductility. The foil was then cold rolled to approximately $225 \mu \mathrm{m}$, annealed a second time, and finally rolled to a thickness of $175 \mu \mathrm{m}$ before undergoing a final 2 hour $630^{\circ} \mathrm{C}$ heat treatment. A $4 \mathrm{~cm}$ strip across the 15 $\mathrm{cm}$ width of the final foil was sectioned into three $4 \times 5 \mathrm{~cm}$ samples for diffraction analysis. Three $2 \mathrm{x}$ $2 \mathrm{~cm}$ samples were also sectioned from the center width of the foil, mounted, polished, and imaged with polarized light along the three orthogonal directions (RD-rolling, TD-transverse, ND-normal) of the foil to establish the microstructure. 
A Panalytical X'pert PRO powder diffractometer equipped with an open Eulerian cradle was used to measure the texture of each of the three diffraction samples using both $\mathrm{Cu} \mathrm{K \alpha}$ and Mo $\mathrm{K} \alpha$ source tubes. In both cases the diffractometer was arranged in point focus mode, with $2 \mathrm{~mm} \times 1 \mathrm{~mm}$ slits to define the cross-section of the beam. The diffracted beam optics consisted of a parallel plate collimator with a $0.18^{\circ}$ acceptance angle. $\mathrm{Ni}$ and $\mathrm{Zr}$ filters were used to remove $\mathrm{Cu} \mathrm{K} \beta$ and $\mathrm{Mo} \mathrm{K} \beta$ radiation, respectively. The samples were tilted up to $80^{\circ}$ and rotated a full $360^{\circ}$, both in $5^{\circ}$ steps. This experimental setup obviated the need to employ any defocusing corrections, which was confirmed by tests on a randomly textured polycrystalline $\alpha$-Ti standard utilizing the same diffraction alignment and settings. Prior to measurement, the samples were pickled with nitric acid to remove the oxide layer which had accumulated on their surfaces during storage, rinsed with methanol, and wiped with lens cleaning paper. Pickling removed less than $1 \%$ of the foil mass, and therefore less than $1 \mu \mathrm{m}$ from each surface.

Bulk foil textures were subsequently acquired via neutron diffraction utilizing the HIPPO time-of-flight (TOF) instrument located at the Los Alamos Neutron Science Center (LANSCE) within the Los Alamos National Laboratory. 1200 Helium-3 detector tubes are arranged on 45 panels in 5 separate rings surrounding the sample. Each detector measures the intensity of diffracted neutrons and, independently, the time it takes for these neutrons to traverse the fixed distance from the source to the detector (Los Alamos National Laboratory, 2015; Matthies et al., 2005; Wenk et al., 2003). The diffracted neutron velocities and, in turn, the wavelengths are then calculated from this temporal data at each detector location. The TOF method utilizes a polychromatic "white" pulsed neutron source with wavelengths covering more than 100 diffraction peaks for $\alpha$-uranium. The detector arrangement is designed to simultaneously optimize spatial coverage and resolution so that, with full diffraction spectra being measured concurrently at over a thousand locations in 45 detector panels around the sample, the complete orientation distribution function (ODF) can be determined from simultaneous Rietveld analysis with only a few sample rotations. The $\alpha$-uranium foil samples were mounted in a single-axis rotating goniometer on the HIPPO robotic sample changer (Losko et al., 2014) and measured under rotations about $\mathrm{TD}$ of $-45^{\circ}, 22.5^{\circ}$, and $45^{\circ}$ (with the beam parallel to ND at $0^{\circ}$ ) for 15 minutes each. 


\subsection{Results}

Metallographic analysis of the $\alpha$-uranium foils was performed via polarized optical microscopy, with the thin film oxide layer $(<<1 \mu \mathrm{m})$ that forms on the polished surface providing intergranular contrast (Figure 4.2). As-cast $\alpha$-uranium has a grain size of 2-3 mm (Yemel'yanov et al., 1969), which can be refined significantly through hot/cold working and recrystallization annealing, reducing the average grain size to between $10 \mu \mathrm{m}$ and $100 \mu \mathrm{m}$ (McCabe et al., 2015; Yemel'yanov et al., 1969). On the basis of the linear intercept method (E112-13, ASTM Standard, Standard Test Methods for Determining Average Grain Size, 2013), the average grain diameter in the foil is approximately $10 \mu \mathrm{m}$, which is consistent with the extensive deformation and multiple recrystallization treatments undergone by the foils. Grains in the foil are equiaxed along all three orthogonal sample directions and there is a low density of deformation twins, despite the fact that plastic deformation generally leads to heavy twinning in $\alpha$-uranium (McCabe et al., 2015, 2010). This confirms that the final heat treatment, and likely those prior, led to complete recrystallization of the microstructure. Precipitates related to carbon impurities are observed in bands along the rolling direction of the foils.

(a)

(b)

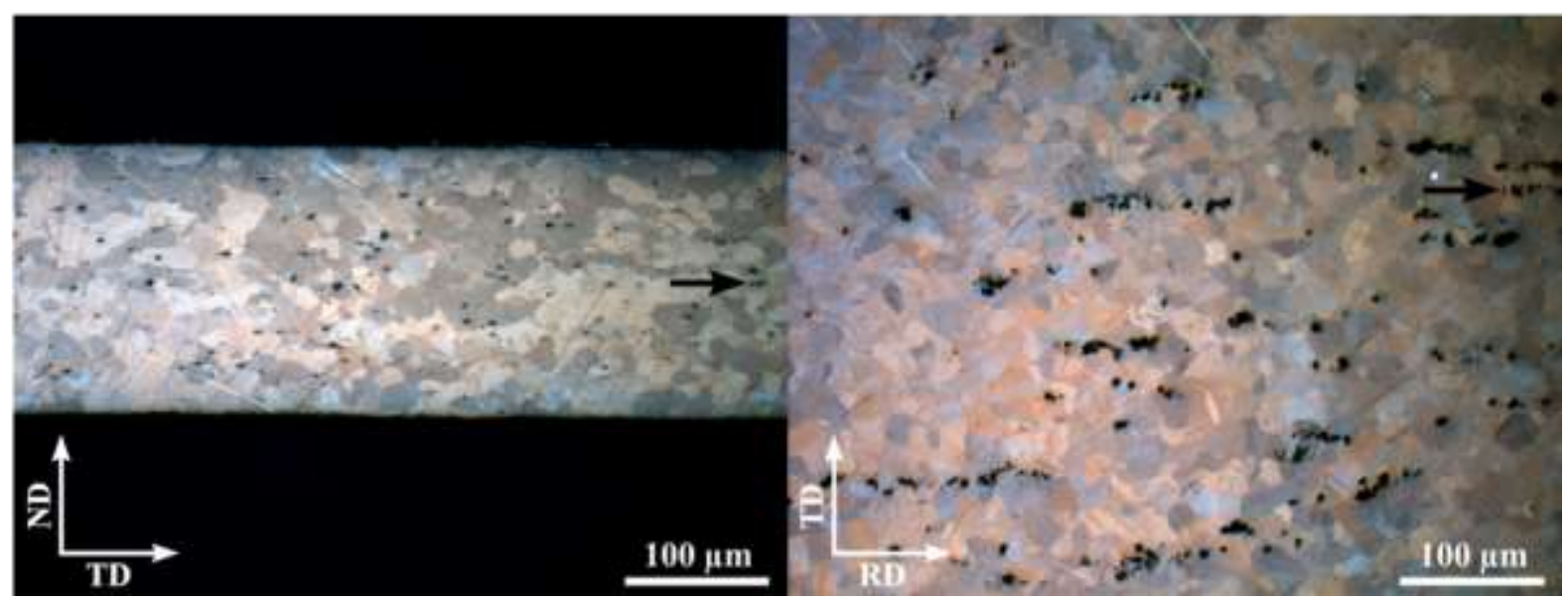

Figure 4.2 Polarized optical micrographs of the $\alpha$-uranium foil for (a) view along RD and (b) view along ND, showing equiaxed $10 \mu \mathrm{m}$ diameter grains and bands of precipitates (noted with black arrows) along the rolling direction that are related to carbon impurities. 
Neutron diffraction data from HIPPO comes in the form of full spectra acquired at hundreds of different detector locations surrounding the sample. These spectra are fit using a Rietveld refinement procedure, which is implemented by the texture analysis software package, MAUD, and corrects for instrument and material-dependent errors. The fitted patterns are then used to compute a full (non-symmetrized) ODF with the E-WIMV algorithm, which discretizes Euler space into a regular grid (e.g. $10^{\circ}$ resolution) and assigns an intensity value to each grid point based on the data (Matthies, 2002; Matthies et al., 2005; Wenk et al., 2003, 1994). For both X-ray sources the nonsymmetrized ODFs were computed using MTEX (Bachmann et al., 2010) with $5^{\circ}$ resolution, and for consistency MTEX was used to display all pole figures presented in this paper. Averages across the three samples were computed and used to calculate pole figures, presented as equal area projections in units of multiples of a random distribution (MRD). No differences in texture were observed between the samples for a given radiation source, even though they were sectioned from different locations along the width of the foil. The average texture over the entire foil, as measured by neutron diffraction, is presented in Figure 4.3. While the ODF for the neutron measurement is computed using all 100 measured pole figures, no changes to the texture are observed by including more than the first eight pole figures in the calculation. For direct comparison with the X-ray diffraction (XRD) measurements, where the number of available pole figures is more limited, the ODF was also calculated using only the three most accessible peaks of $\alpha$-uranium (strongest intensities with minimal overlaps), i.e. (002), $\{111\}$, and $\{112\}$. This mock-XRD measurement closely approximates the larger dataset (Figure 4.3(b)), with the primary difference being a slight reduction in the texture strength. Texture strength can be represented quantitatively by the texture index JODF that approaches 1 for a completely random orientation distribution, and infinity for a perfect single crystal (Mainprice et al., 2014). The texture index for the complete neutron texture in Figure 4.3 is JODF $=2.43$, while the texture using only the three peaks weakens to JODF $=1.97$. 


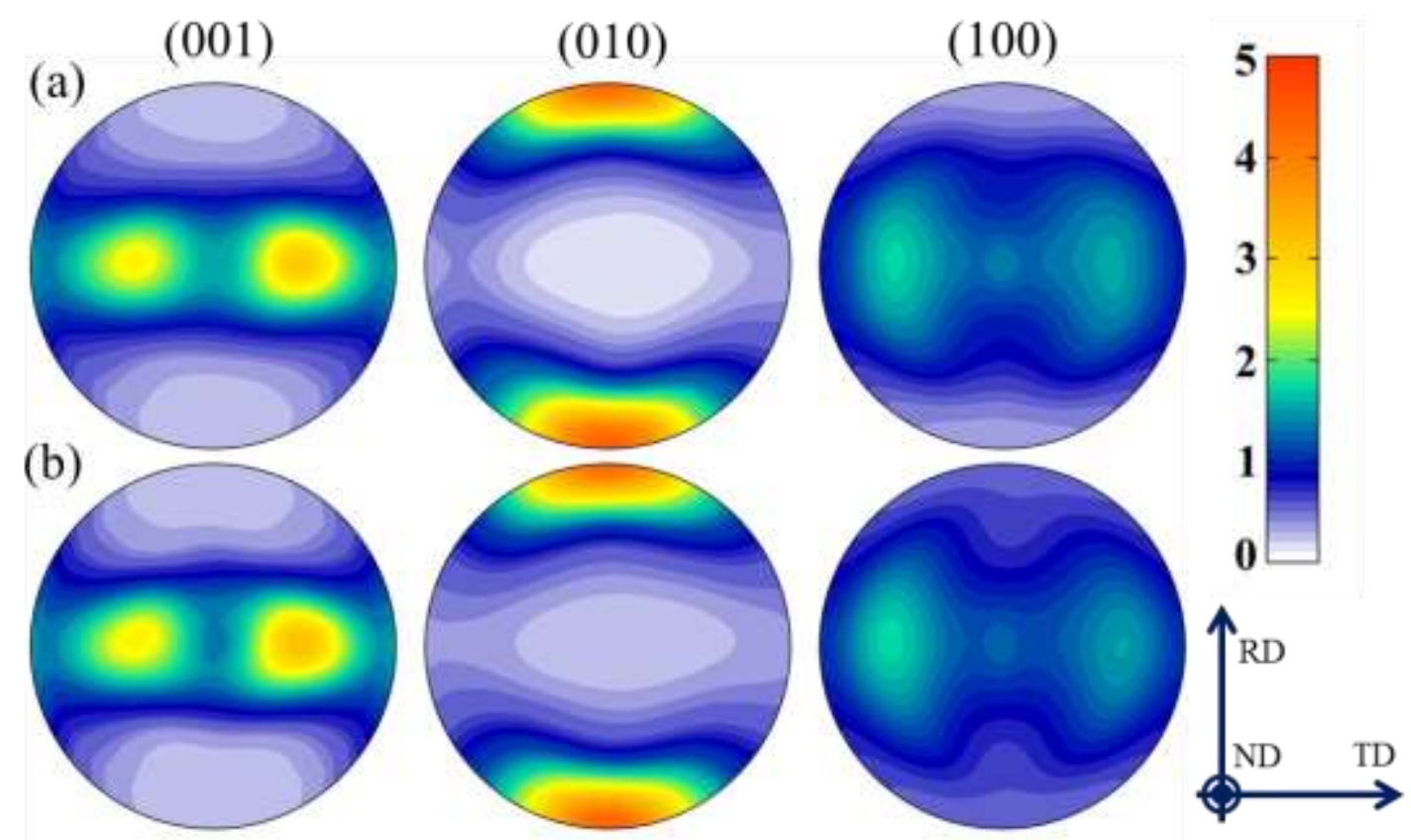

Figure 4.3 Pole figures from the calculated ODF of the $\alpha$-uranium foil bulk texture measured by neutron diffraction, using (a) the full complement of 100 measured diffraction peaks or (b) only the (002), $\{111\}$, and $\{112\}$ peaks to compute the ODF.

Three experimental pole figures is the theoretical lower limit necessary to calculate an ODF from a sample with orthorhombic crystal symmetry, so no further reduction is possible (Bunge, 1982). Comparing the neutron texture computed with a reduced number of peaks (Figure 4.3(b)) to that calculated using the full dataset (Figure 4.3(a)), the similarity suggests that only experimental pole figures of these three peaks are necessary to adequately characterize $\alpha$-uranium rolling textures, despite the orthorhombic crystal structure having a lower symmetry than cubic. The bulk texture of the $\alpha$-uranium foil exhibits a strong alignment of the (010) poles along RD, with some tangential spread along the edge of the pole figure (rotation about ND) and a clear absence of (010) poles near the TD-ND plane. In contrast the (001) poles reside almost exclusively in the TD-ND plane, with two high intensity peaks split along TD away from the ND axis. The (100) poles are nearly randomly distributed but have two broad lobes of intensity in a position similar to the stronger (001) TD split features.

Experimental pole figures of the (002), $\{111\}$, and $\{112\} \alpha$-uranium diffraction peaks were acquired using both X-ray sources. These three high intensity peaks do not significantly overlap with other $\alpha$-uranium peaks or those of $\mathrm{UO}_{2}$ and, as demonstrated in Figure 4.3, are sufficient to 
characterize texture of $\alpha$-uranium. Background intensity pole figures were also measured near each diffraction peak and subtracted from the raw pole figure data. The experimental partial pole figures were truncated beyond $70^{\circ}$ or $75^{\circ}$ tilt to fully eliminate minor artifacts attributed to defocusing, and complete ODFs were computed using MTEX. Figure 4.4 presents the texture of the $\alpha$-uranium foil surface measured with $\mathrm{Cu} \mathrm{K \alpha}$ X-rays. The surface texture of the foil is qualitatively similar to the bulk texture but is slightly stronger $(\mathrm{JODF}=3.15)$ and there are some notable deviations, best observed in a difference pole figure (Figure 4.5). The (010) poles exhibit a strong alignment along RD that is comparable to the bulk texture, but there is a ring of weak intensity around the edge of the entire pole figure which is not present in the bulk of the foil. In the (001) pole figure the intensity is no longer spread across the entire TD-ND plane and resides almost solely in the split TD intensity elements, which are stronger than in the bulk. In addition, a weaker RD split element that is not present in the bulk foil texture is now observed in the (001) pole figure. The two TD split lobes found in the (100) pole figure of the bulk texture are moderately stronger in the surface texture, and have broadened considerably along the tangential direction to form a ring. It is worth noting the ring-shaped intensity features in the (010) and (100) pole figures are not an artifact of the X-ray methodology, as similar features appear in electron backscattered diffraction (EBSD) measurements (Zecevic et al., 2016a, 2016b).

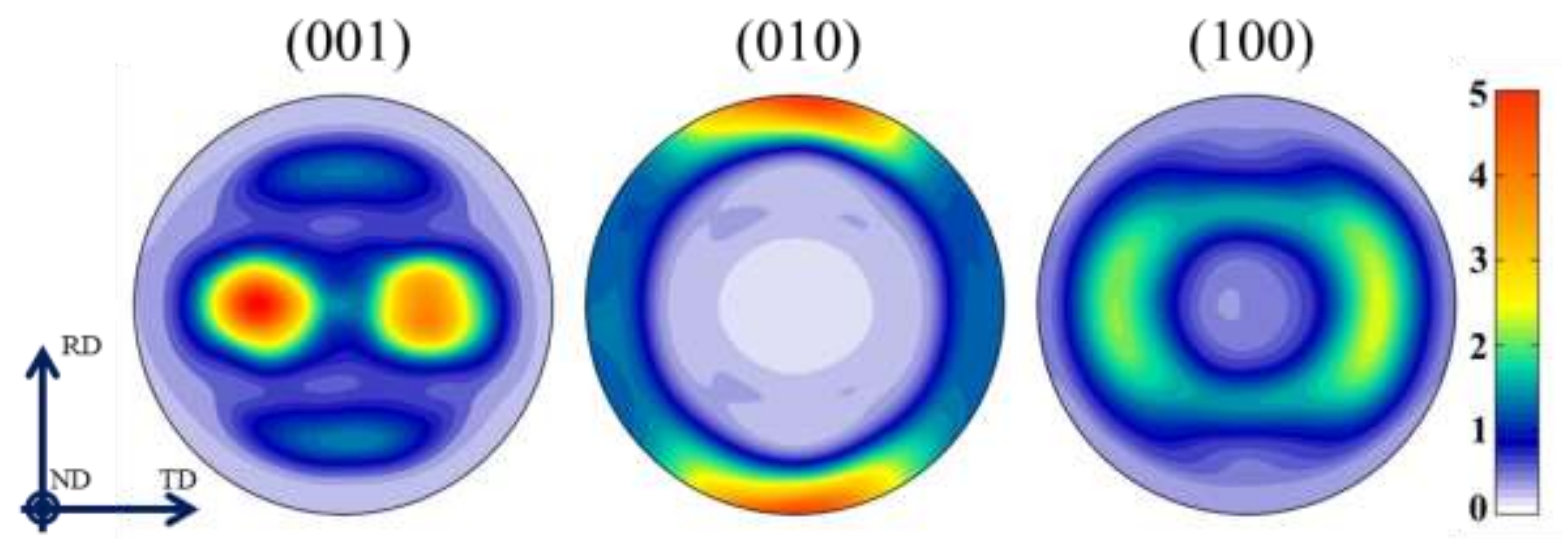

Figure 4.4 Pole figures from the calculated ODF of the $\alpha$-uranium foil surface texture measured using $\mathrm{Cu} \mathrm{K} \alpha \mathrm{X}$ rays and the (002), $\{111\}$, and $\{112\}$ peaks. 

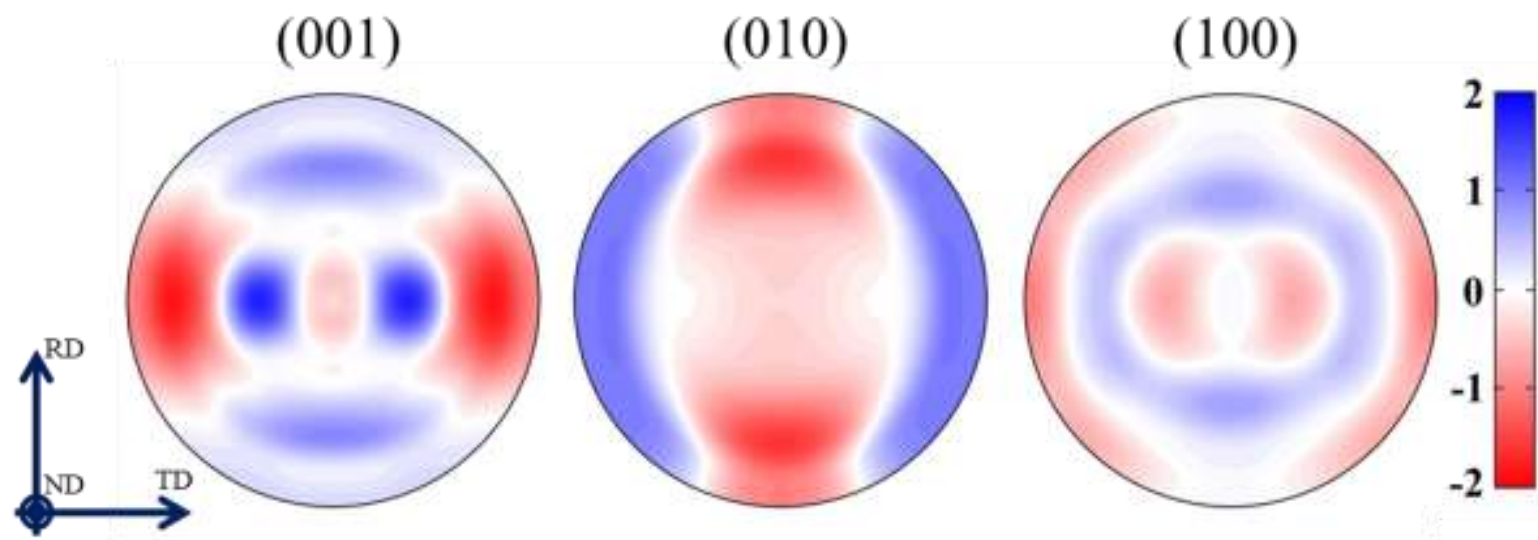

Figure 4.5 Pole figures of the difference in MRD intensity between the surface (Figure 4.4) and bulk (Figure 4.3) texture measurements. The rolled foil's orthotropic sample symmetry has been imposed to smooth experimental variations in intensity and better clarify trends.

The experimental pole figures acquired using Mo K $\alpha$ suffered in quality due to X-ray fluorescence. Mo $\mathrm{K} \alpha$ radiation $(\mathrm{K} \alpha 1=17.479 \mathrm{keV}, \mathrm{K} \alpha 2=17.374 \mathrm{keV})$ is located just above the $\mathrm{L}_{\text {III }}$ absorption edge for uranium $(17.166 \mathrm{keV})$ and consequently it was found that it leads to a substantial amount of fluorescence that obscures the diffraction peaks (Bearden, 1967). This is further compounded by the large atomic number of uranium $(Z=92)$, as the fluorescent yield (production of X-rays over Auger electrons) is higher than most materials (Krause, 1979). Therefore, the background signal is 5.5 times higher using Mo $\mathrm{K} \alpha$ as opposed to $\mathrm{Cu} \mathrm{K} \alpha$, and the measured texture is substantially weaker $\left(\mathrm{J}_{\mathrm{ODF}}=1.50\right)$. Nonetheless, the texture measurement provided by the Mo K $\alpha$ source confirms several of the texture features observed in the $\mathrm{Cu} \mathrm{K} \alpha$ surface texture, and that these features may extend past the first micrometer of the foil's surface (Figure 4.6). The texture component between ND and $\mathrm{RD}$ in the (001) pole figure is stronger than that measured using $\mathrm{Cu} \mathrm{K \alpha} \mathrm{X-rays,} \mathrm{and} \mathrm{intensities} \mathrm{in}$ the (010) pole figure are notably weaker than for either of the other measurements. Interestingly, the (100) pole figure has a four-fold symmetric element instead of having its strongest feature centered along the TD-ND plane. While the Mo Ka texture is informative, given the lower fidelity of data, the $\mathrm{Cu} \mathrm{K} \alpha$ measurement is likely most representative of the foil surface texture. 


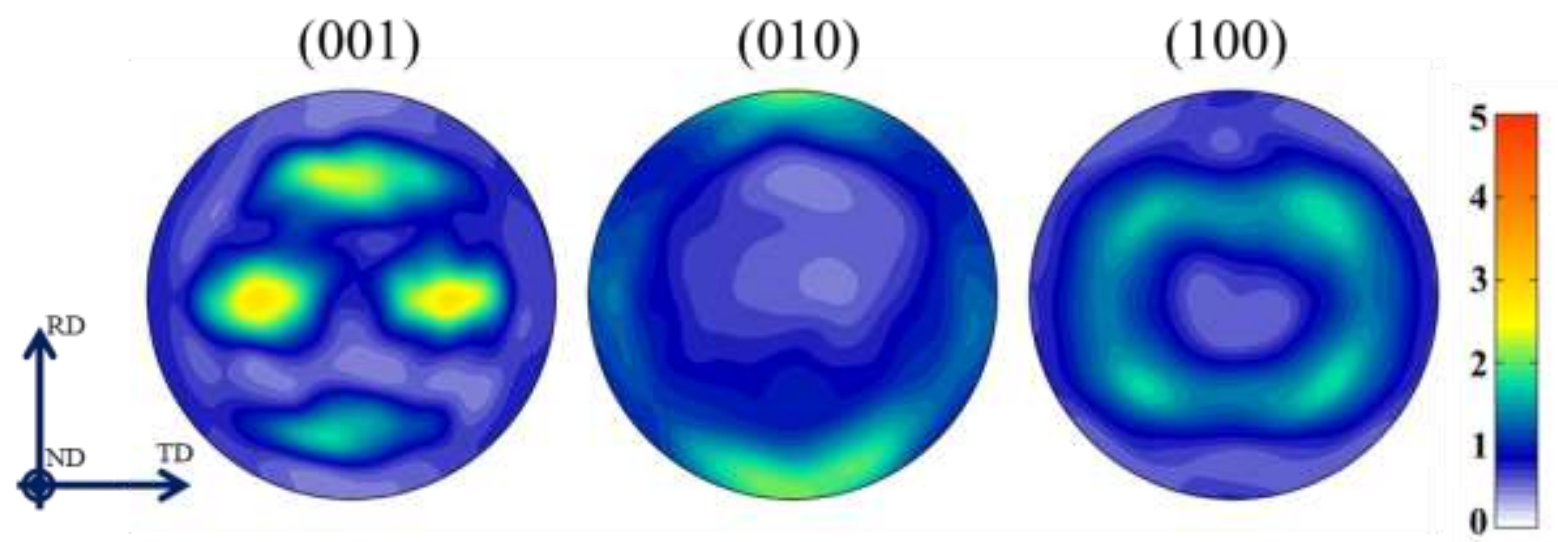

Figure 4.6 Pole figures from the calculated ODF of the $\alpha$-uranium foil surface texture measured using Mo K $\alpha$ $\mathrm{X}$-rays and the (002), $\{111\}$, and $\{112\}$ peaks.

The possibility that the (002) diffraction peak intensity could be influenced by the tail of the nearby $\{021\}$ peak in the XRD measurements was considered and does not appear to be a factor in the difference between the surface and bulk measurements. Using the bulk neutron data, where the problem of peak overlap is mitigated by analyzing the full pattern via Rietveld refinement (Lutterotti et al., 1997), a test was performed by superimposing some of the $\{021\}$ pole figure intensity onto the (002) pole figure before calculating the ODF. The changes observed in this simulated texture are decidedly different than those found in Figure 4.5, suggesting that the tail of the $\{021\}$ peak does not significantly impact the XRD texture measurement.

\subsection{Discussion}

The bulk texture of the foils (Figure 4.3) is generally consistent with that observed in recrystallized warm straight-rolled $\alpha$-uranium plates (Knezevic et al., 2012; Lloyd and Mueller, 1962). The warm straight-rolled texture is marked by a strong $\mathrm{RD}$ alignment of the (010) poles, and ND alignment of the (001) poles with a broad spread along TD. It does not, however, exhibit the full TD splitting of the (001) poles away from ND observed in the foils, and the strongest features of the (100) pole figure are located squarely along TD. Curiously, the texture of the foils is much closer, and nearly identical, to that recently reported for recrystallized swaged $\alpha$-uranium (Knezevic et al., 2012). The swaged $\alpha$-uranium texture maintains the strong RD alignment of the (010) poles, while the (001) poles are spread broadly within the TD-ND plane with a clear TD splitting of the most intense 
elements, and the (100) features have rotated off the edge of the pole figure toward ND. The foil surface texture, with the exception of the split RD features in the (001) pole figure, is similar to the recrystallized warm clock-rolled texture of $\alpha$-uranium (Knezevic et al., 2013b, 2012; Steiner et al., 2017); with the (001) poles condensed into two strong TD split elements, and extensive tangential broadening of the (001) features.

The deformation modes active in $\alpha$-uranium are complex and highly dependent on temperature and nature of the applied stress (Brown et al., 2009; Calhoun et al., 2015; Knezevic et al., 2013a, 2013b, 2012; McCabe et al., 2010), so it is not surprising that the cold straight-rolled $\alpha$ uranium foil texture varies from the warm straight-rolled $\alpha$-uranium plate texture. Additionally, recrystallization of $\alpha$-uranium can lead to texture evolution that is dependent on both the deformed state and annealing temperature (Lloyd and Mueller, 1962; Steiner et al., 2017). The texture of deformed (i.e. non-recrystallized) straight-rolled $\alpha$-uranium (Lloyd and Mueller, 1962; Zecevic et al., 2016a, 2016b) is notably different than that of the recrystallized state. In particular, there is a strong feature split from ND along RD in the (001) pole figure, and only a much weaker feature split along TD. The alignment of the (010) poles along RD is also considerably weaker than in the recrystallized state. Recrystallization (above $450^{\circ} \mathrm{C}$ ) results in the disappearance of the RD split intensity in (001) in favor of a strengthened TD split element, and reinforcement of the (010) pole alignment along RD (Lloyd and Mueller, 1962; Steiner et al., 2017). Simulations and experimental results show that the deformation texture of $\alpha$-uranium varies from the surface to the mid-plane during straight-rolling (Zecevic et al., 2016b). This manifests itself primarily as asymmetry in the (001) pole figure, with the large shear stress at the sample surface resulting in one of the RD split features becoming much stronger than the other. This asymmetric surface texture is likely to evolve differently than the symmetric bulk deformation texture during recrystallization and will presumably produce a texture similar to that observed at the foil surface. The four-fold symmetric feature observed in the (100) Mo K $\alpha$ pole figure appears in some straight-rolled texture studies (Lloyd and Mueller, 1962) but not others (Knezevic et al., 2012), and this feature is believed to be heavily dependent on specific recrystallization conditions (Steiner et al., 2017). 


\subsection{Conclusions}

The texture of recrystallized cold straight-rolled $\alpha$-uranium foils has been measured using both neutron and X-ray diffraction, as the different penetration depths of the two methods allow for comparison of the bulk and surface textures. While the bulk $\alpha$-uranium foil texture is similar to that of recrystallized warm straight-rolled $\alpha$-uranium plates, there are notable differences, including a split of the strong (001) pole alignment along TD. The surface texture exhibits an additional RD split element in the (001) poles, believed to arise from shear deformation during rolling, though an incomplete understanding of texture evolution in $\alpha$-uranium during recrystallization obscures a direct conclusion. It is demonstrated through neutron diffraction that only the (002), $\{111\}$, and $\{112\}$ experimental pole figures are necessary to satisfactorily establish $\alpha$-uranium rolling textures, making it possible to produce textures of comparable quality using X-ray diffraction.

\subsection{Acknowledgements}

The authors would like to thank Amy DeMint (Y-12) for her help preparing the samples for this study. Funding for this research was provided by the Y-12 National Security Complex under the Plant Directed Research, Development, and Demonstration program. This work of authorship and those incorporated herein were prepared by Consolidated Nuclear Security, LLC (CNS) as accounts of work sponsored by an agency of the United States Government under Contract DE NA 0001942. Neither the United States Government nor any agency thereof, nor CNS, nor any of their employees, makes any warranty, express or implied, or assumes any legal liability or responsibility to any nongovernmental recipient hereof for the accuracy, completeness, use made, or usefulness of any information, apparatus, product, or process disclosed, or represents that its use would not infringe privately owned rights. Reference herein to any specific commercial product, process, or service by trade name, trademark, manufacturer, or otherwise, does not necessarily constitute or imply its endorsement, recommendation, or favoring by the United States Government or any agency or contractor thereof, or by CNS. The views and opinions of authors expressed herein do not necessarily state or reflect those of the United States Government or any agency or contractor (other than the 
authors) thereof. This document has been authored by Consolidated Nuclear Security, LLC, under Contract DE NA 0001942 with the U.S. Department of Energy/National Nuclear Security Administration, or a subcontractor thereof. The United States Government retains and the publisher, by accepting the document for publication, acknowledges that the United States Government retains a nonexclusive, paid up, irrevocable, worldwide license to publish or reproduce the published form of this document, prepare derivative works, distribute copies to the public, and perform publicly and display publicly, or allow others to do so, for United States Government purposes. 


\section{Crystallographic Texture Measurement via Neutron Diffraction at the Oak Ridge National Laboratory}

\section{Attribution:}

The following text is taken from a manuscript prepared for submission to The Journal of Applied Crystallography in July of 2017. X-ray texture measurements and initial analysis using MTEX were performed by Dr. Jishnu Bhattacharyya at the University of Virginia. Neutron diffraction measurements at the Neutron Residual Stress Mapping Facility (NRSF2) were performed and analyzed preliminarily by Dr. Chris Fancher, Dr. Jeff Bunn and John Einhorn at the High Flux Isotope Reactor (HFIR), Oak Ridge National Laboratory (ORNL). They were further analyzed with MTEX by John Einhorn and Dr. Fancher. Neutron diffraction measurements at the Nanoscale Ordered Materials Diffractometer (NOMAD) were performed and analyzed preliminarily by Dr. Matthew Tucker and John Einhorn at the Spallation Neutron Source (SNS), ORNL. They were further analyzed with MAUD and MTEX by John Einhorn. The data reduction procedure for texture analysis using NOMAD was conceived of, implemented and documented by John Einhorn, with the expert advice of Dr. Peter Peterson (ORNL) and others. The literature review, writing and preparation for the manuscript was performed by John Einhorn. All authors contributed to editing before initial submission. Drs. Sean Agnew and Andrew Payzant provided invaluable direction and insight to the project at all stages. An exhaustive list of acknowledgements, mostly concerning work conducted at the ORNL, is given at the end of the chapter. 


\title{
Crystallographic Texture Measurement via Neutron Diffraction at the Oak Ridge National Laboratory
}

\author{
J.R. Einhorn ${ }^{\text {*, C.M. Fancher }}{ }^{2}$, M. Tucker ${ }^{2}$, J.Bhattacharyya ${ }^{1}$, J.R. Bunn ${ }^{2}$, and S.R. \\ Agnew $^{1}$ \\ ${ }^{1}$ University of Virginia, Material Science and Engineering, 395 McCormick Rd, Charlottesville, VA 22904, USA \\ ${ }^{2}$ Oak Ridge National Laboratory, Oak Ridge, TN 37831, USA \\ *Corresponding Author: jre8sy@virginia.edu
}

\subsection{Abstract}

The capability to make efficient and reliable texture measurements using the Second Generation Neutron Residual Stress Mapping Facility (NRSF2) within the High Flux Isotope Reactor (HFIR) and Nanoscale Ordered Materials Diffractometer (NOMAD) at the Spallation Neutron Source (SNS), both at the Oak Ridge National Laboratory (ORNL), is demonstrated. The texture of an Al alloy sample was measured on both instruments and compared with X-ray diffraction (XRD) measurements. The data from NOMAD was processed using the E-WIMV and harmonic texture algorithms in the Materials Analysis Using Diffraction (MAUD) program, whereas the data obtained using XRD and on NRSF2 were analyzed using the MTEX texture toolbox within MATLAB. The three datasets (XRD, NRSF2 and NOMAD) were compared, and reasonable agreement was found across the methods. However, some differences in overall texture strength and the strength of some components were identified. The texture data obtained on NRSF2 and NOMAD, analyzed using the E-WIMV algorithm, agree best. The differences are attributed mostly to the differences in the corresponding analysis approaches inherent to each measurement technique. The differences are also compared to differences between results of crystal plasticity-based texture simulations in the literature and their impact on polycrystalline property prediction is discussed. Finally, suggestions for future work on both instruments are presented, and it is proposed that the measurement time can be significantly reduced to about 9 hours/sample and 20 minutes/sample for NRSF2 and NOMAD, respectively. 


\subsection{Introduction}

\subsubsection{Motivation}

Preferred crystallographic orientation, or "texture," causes many polycrystalline materials to exhibit anisotropy in their properties, and models can be developed to predict such structure-property relationships (Kocks et al., 1998). Additionally, processing-structure relationships can be investigated by varying the processing history and measuring the resulting texture. With this suite of information in hand, engineers can design manufacturing processes to yield textures that result in desirable properties for the application of interest. Texture is also of interest in the study of geological materials, which tend to develop texture due to "processing" conditions within the Earth's crust, thereby yielding information on the thermomechanical history of the material.

Neutrons interact relatively weakly with matter, allowing for much higher penetration depths than X-rays and electrons, which gives neutron diffraction several advantages over traditional methods for texture measurement (Bunge, 1989). Firstly, diffraction occurs at depth within samples, yielding texture information for the bulk material rather than the surface alone. This allows for large samples to be measured nondestructively, which is generally not possible with other methods and is critical when specimen preparation is difficult, undesirable or impossible (e.g. mineral samples or engineering assemblies) (Kockelmann et al., 2006b). A second consideration is that the diffracting gauge volume is significantly larger with neutrons, leading to better grain statistics and thereby allowing for robust measurement, especially of large-grained samples. Finally, since obstruction of the beam is less significant for neutrons, larger and more sophisticated sample environments can be employed. This allows for in situ texture studies (provided that the time resolution required is not too small) under various non-ambient conditions (e.g. high temperature or pressure) and for highthroughput instruments using automated sample-changing devices (Reiche et al., 2015).

The Second Generation Neutron Residual Stress Mapping Facility (NRSF2) within the High Flux Isotope Reactor (HFIR) is typically employed for bulk residual stress mapping of engineering materials (Hempel et al., 2016) and the Nanoscale Ordered Materials Diffractometer (NOMAD) at the Spallation Neutron Source (SNS) is typically employed for Pair Distribution Function (PDF) analysis 
of liquids and amorphous solids (Neuefeind et al., 2012). The goal of this study is to demonstrate that both of these instruments can be used to measure texture reliably. To that end, results are presented from each instrument and compared to each other, and to those obtained via traditional using X-ray diffraction (XRD ) based pole figure measurement. The study will also highlight the relative advantages and disadvantages of each instrument, helping future users make an informed choice of instrument for texture measurement at the Oak Ridge National Laboratory (ORNL).

\subsubsection{Comparison of Methods}

Though traditional pole figure methods employing monochromatic radiation and angulardispersive point detection lack the efficiency of multi-detector time-of-flight (TOF) methods, they yield data that can be easier to analyze and interpret. Moreover, for materials with sharp or known texture, high crystallographic symmetry, and/or only one phase, the additional information is typically unnecessary, and pole figure measurement can be stream-lined to approach similar measurement timescales (Wenk et al., 2001). The open layout on the NRSF2 makes it ideal for measuring large samples, such as engineering assemblies.

NRSF2 is well-equipped to perform angular-dispersive texture measurements, since it can be configured with a 2-circle goniometer capable of performing the requisite rotations quickly and precisely (Bunn et al., 2014). Additionally, the diffractometer has seven ${ }^{3} \mathrm{He}$ detectors, which allow for greater coverage of orientation space and thus greater time efficiency for pole figure measurement. A principal advantage of this technique is that exceedingly large or oddly-shaped samples can be measured non-destructively. A similar measurement technique was employed on STRESS-SPEC at FRM-II, an instrument similar to NRSF2 except that it uses a single 2-D position-sensitive detector (Brokmeier et al., 2011).

Time-of-flight (TOF) neutron instruments allow for efficient diffraction measurements, and this technique is routinely used to measure texture on instruments known as HIPPO (High-PressurePreferred Orientation) at Los Alamos (Los Alamos National Laboratory, 2015; Wenk et al., 2003), GEM at ISIS (Kockelmann et al., 2006a), SKAT at Dubna (Ullemeyer et al., 1998), and iMATERIA at J-PARC (Onuki et al., 2016). Nevertheless, increasing the availability is of interest, especially to 
partner with other in situ measurements on the same samples using VULCAN at the SNS (c.f. Kim et al. 2017), but is not quite as efficient as NOMAD specifically for texture (Ma et al., 2017). NOMAD is an ideal candidate because it employs ${ }^{3} \mathrm{He}$ detectors arranged in banks at various diffraction angles $2 \theta$ and that surround the sample in rings (Neuefeind et al., 2012). This is advantageous for texture measurement because it allows the volume fractions of many grain orientations to be measured simultaneously, significantly reducing measurement time with respect to traditional pole figure methods. Owing to good detector coverage of orientation space, only 3 to 4 in-plane sample rotations are generally required to fully determine an orientation distribution (OD) on HIPPO, resulting in about $1 \mathrm{hr}$. total measurement time per sample (Wenk et al., 2010). This huge gain in time efficiency is the most important advantage of measuring texture using TOF instruments, including NOMAD. The main disadvantage of NOMAD is that only samples with a certain size and shape can be measured at present, meaning that sectioning is required for large samples.

Texture data has been collected on HIPPO for a wide variety of materials (Gómez Barreiro et al., 2015; Matthies et al., 2005; Vogel et al., 2004; Wenk et al., 2003). The software MAUD is used to perform Rietveld texture analysis (RITA), which fits the diffraction spectra recorded at each detector (bin) based on structural parameters for the sample, and then uses the intensity variation as a function of orientation (i.e. detector location) to determine the OD (Lutterotti et al., 1997). One goal of this study is to perform this analysis using data from NOMAD.

\subsection{Experimental Methods}

\subsubsection{Choice and Preparation of Specimen and XRD Experiment}

As part of a separate previous study on the effects of aging treatment on the resulting texture of cold-rolled commercial 7xxx-series $\mathrm{Al}$ alloys, the texture through the thickness of a plate sample of AA7085-T721 (cold-rolled and over-aged) was characterized via XRD, according to the experimental procedures outlined in (Bhattacharyya et al., 2017). Specimens were sectioned parallel to the plane of the rolling direction (RD) and transverse direction (TD) at intervals along the normal direction (ND), including at the center of the plate ("mid-plane"; $\sim 20 \mathrm{~mm}$ from surface). The approximate dimensions 
of the mid-plane XRD specimen, which was mechanically polished prior to measurement, were $20 \mathrm{x}$ $20 \times 3 \mathrm{~mm}(\mathrm{RD} \times \mathrm{TD} \times \mathrm{ND})$.

For the neutron experiments, a cylindrical specimen of diameter $7.3 \mathrm{~mm}$ and length $20 \mathrm{~mm}$ was electric discharge machined (EDM) from the center of the plate, with the cylinder axis parallel to the RD. The center of the plate was chosen because the texture is known from the XRD results to be most uniform along ND there. The cylindrical specimen geometry was chosen to be suitable for both NRSF2 and NOMAD, the latter of which commonly employs tube-shaped sample cans. The geometry is also advantageous (relative to a cuboidal specimen) from the perspective of path length isotropy, though the effect of path length differences is negligible for a low neutron absorber like $\mathrm{Al}$ (Sears 1992). The specimen was marked to facilitate its orientation with respect to the beam.

\subsubsection{NRSF2}

\subsubsection{Data Collection}

The measurement strategy on NRSF2 is quite similar to the standard X-ray pole figure measurement approach. At each diffraction angle $2 \theta_{h k l}$, the sample is tilted and rotated at each tilt so that the diffraction vector $\boldsymbol{Q}=\Delta \boldsymbol{k}$ maps a diffraction pole hemisphere (of which the pole figure is a projection) around the initial sample orientation. This is slightly more efficient than the standard approach: since the detector array extends out of the plane of the diffractometer circle, only three outof-plane $\left(\chi=0^{\circ}, 30^{\circ}, 60^{\circ}\right)$ tilts are necessary to cover the hemisphere with roughly $5^{\circ}$ resolution (the detector centers are $6.5^{\circ}$ apart in $\left.\chi\right)$. The full complement of $\varphi$ rotation $\left(360^{\circ}\right)$ is performed with steps of $5^{\circ}$, for a total of $72 \times 3=216$ collection points. Due to the high penetration depth of neutrons and thus the absence of surface effects, this technique also enjoys the slight advantage over XRD that complete pole figures can be obtained and no defocusing correction is needed. The geometry of the measurement is shown schematically in Figure 5.1. 


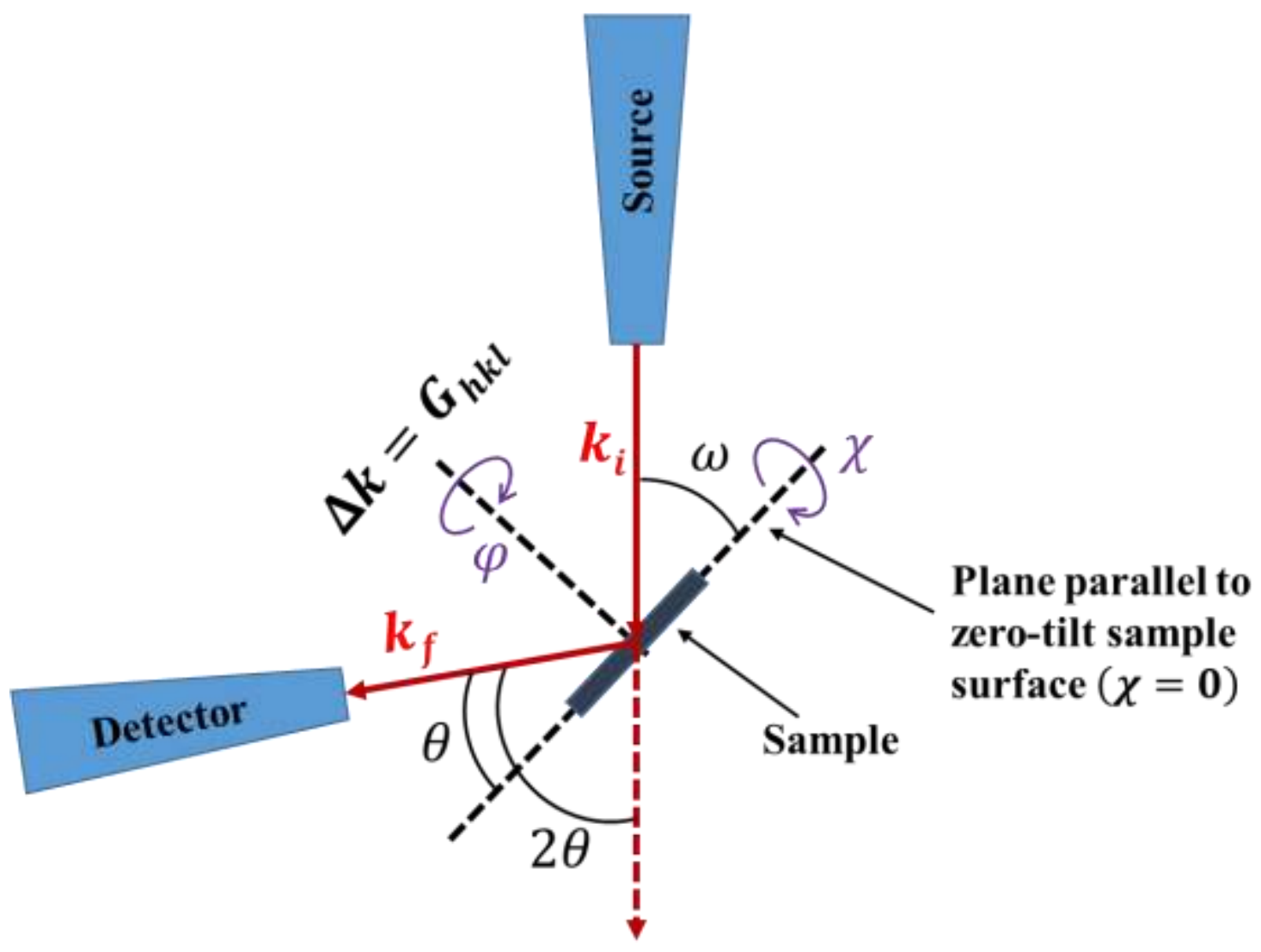

Figure 5.1 Geometry of pole figure measurement on NRSF2. The "detector" is 7 individual detectors that extend out of the page on a circular arc.

Counting time was $120 \mathrm{~s}$ per measurement point (i.e. at each $\chi, \varphi$ ), for a total of 7.2 hours per pole figure. The incident and diffracted slits defined a $3 \mathrm{~mm} \times 3 \mathrm{~mm} \times 3 \mathrm{~mm}$ gauge volume in the specimen. For cubic materials, three complete pole figures are more than sufficient to determine an OD, but because of delays due to data readout and goniometer motion, roughly 1 day of measurement time was required.

Still, however, by far the most significant disadvantage of this technique is the long collection time (around 1 day/sample presently), while the

\subsubsection{Data Analysis}

The raw data comes in the form of intensity vs. diffraction angle $2 \theta$ at each of the 216 goniometer settings $(\chi, \varphi)$. In the analysis, each of the seven detectors is treated separately, giving a total of $7 \times 216=1512$ data points. At each measurement point, the integrated intensity is obtained from fitting the measured diffraction data using a pseudo-Voigt peak shape function. Then, 
experimental pole figures are constructed by assigning to each data point the appropriate $\alpha, \beta$ coordinates, based on the corresponding $\chi, \varphi$ goniometer setting and on which detector it came from, and imported directly into MTEX for further analysis.

To explore the possibility of reducing the measurement time by collecting fewer points, the data were modified in two different ways and re-analyzed. The "reduced" dataset corresponds to the same data but with the last $\chi$ ring $\left(\chi=60^{\circ}\right)$ reduced to $10^{\circ}$ resolution in $\varphi$ (by removing half of the measurements). For the "doubly reduced" dataset, this operation was performed on both of the last two $\chi$ rings $\left(\chi=30^{\circ}\right.$ and $\left.60^{\circ}\right)$. The two modifications correspond to 180 and 144 measurement points, respectively. The three schemes (including the original) are shown in Figure 5.2(b)-(d), with the coverage of the XRD experiment shown in (a) for comparison.

(a)
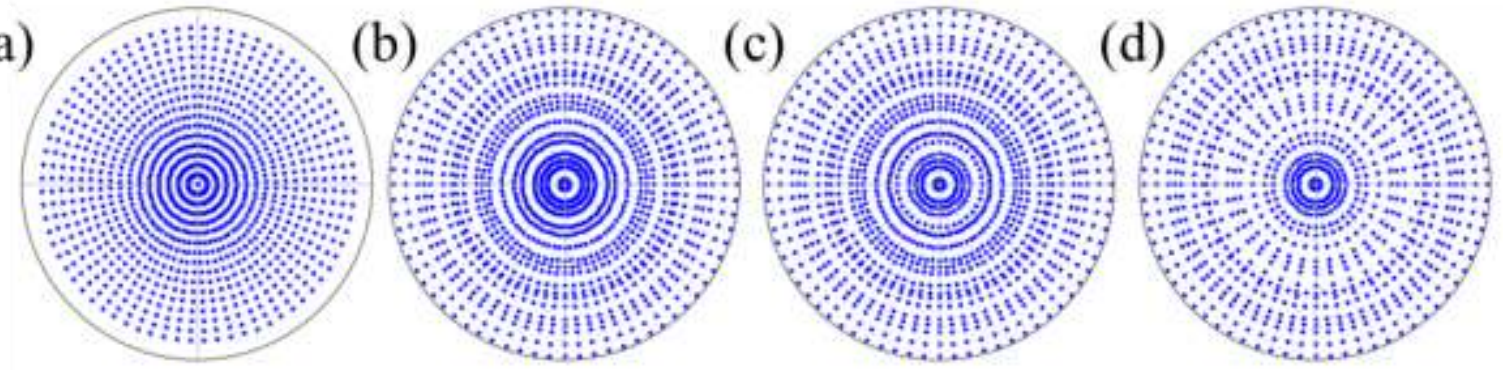

Figure 5.2 Pole figure coverage for (a) XRD $\left(5^{\circ} \times 5^{\circ}\right)$; NRSF2: (b) original $\left(6.5^{\circ} \times 5^{\circ}\right)$, (c) reduced and (d) doubly reduced.

\subsubsection{NOMAD}

\subsubsection{The diffractometer}

Although very similar to HIPPO, NOMAD poses some additional challenges for texture measurement. Firstly, the detector coverage is slightly lower and less uniform. In addition, while the broad TOF range available in the lower $2 \theta$ detector rings $\left(\leq 90^{\circ}\right)$ permits a wide range of d-spacings to be measured at low resolution, the higher $2 \theta$ rings cover a narrow range but at high resolution (Usher, 2016). This limits the usable TOF range and thus the number of pole figures that can be collected with certain detectors. Still, it is possible for each detector to collect $>10$ diffraction peaks even for FCC materials like Al, and many more for lower symmetry materials. 
NOMAD has 50 detector panels arranged on six rings* surrounding the sample (Figure 5.3). Each panel or "8-pack" consists of eight ${ }^{3} \mathrm{He}$ detector tubes, and each tube contains 128 pixels along its length. This gives a total of $50 \times 128 \times 8=51200$ pixel locations, most of which will record usable diffraction spectra for a polycrystalline sample.

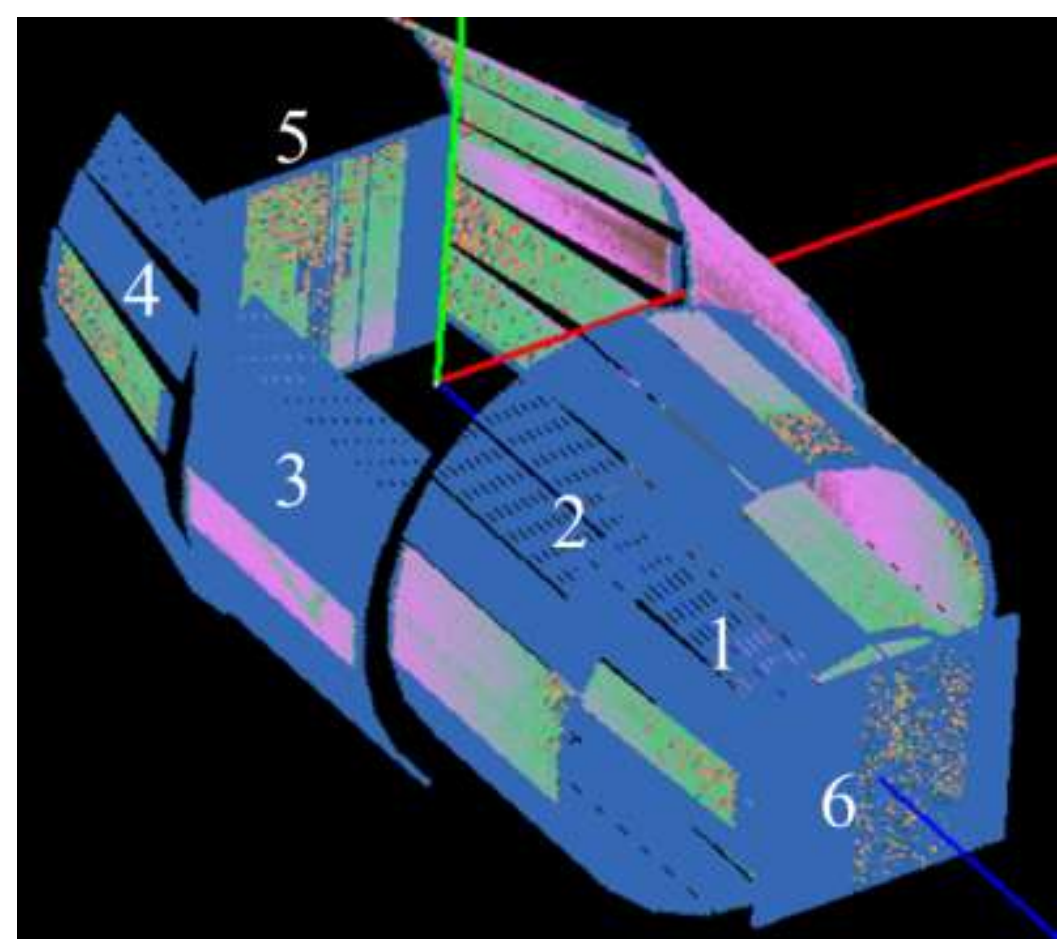

Figure 5.3 The NOMAD detector suite, with active detectors shown in multi-color. The instrument coordinate system is $+Z=$ blue (beam direction), $+Y=$ green, $+X=$ red. The origin is the sample location, and the rings are numbered in white.

When neutron scattering is assumed to be isotropic (e.g. in powder diffraction or PDF analysis), all spectra should be similar, so it is best to group as many together as possible (i.e. fewer groups) for better statistics. Owing to the differences in TOF resolution and range mentioned earlier, the standard NOMAD procedure groups the data by ring, for a total of 6 groups (Neuefeind et al., 2012). However, for texture analysis it is necessary to separate the data from different detector locations, so more bins must be employed. The next simplest choice is to bin by panel, but it has been determined empirically that a better binning strategy uses 65 bins, and most of the bins are made up of

\footnotetext{
" A note on terminology: in the literature, HIPPO's detector "rings" are referred to as "banks," each of which is divided into multiple packs of detector tubes called "panels" (see e.g. Wenk et al. 2010). In Mantid parlance, the rings are called "groups," and the 8-packs are called "banks." For both instruments, the detector tubes themselves are referred to as "detectors" or "tubes." In this thesis, we use the terms "rings," "panels" and "tubes."
} 
four or less detector tubes (half of a panel) because of how the panels are tilted (and thus spread out when mapped onto the pole figure) or because the data from certain tubes is too noisy.

The instrument coordinate system $(\boldsymbol{X}, \boldsymbol{Y}, \boldsymbol{Z})$ is defined as shown in and Figure 5.4. The position $(r, 2 \theta, \eta)$ of each detector is defined by the spherical coordinates (origin centered on the sample) of the tip of the scattering vector $\boldsymbol{Q}$, i.e. the bisector of the incident and diffracted beams, which are given in instrument coordinates by $(0,0,1)$ and $\left(x_{d}, y_{d}, z_{d}\right)$, respectively (the latter corresponds to the instrument coordinates of the $d$ th detector). These positions are known for all detector pixel locations.

Due to Friedel's Law, the pole sphere is centrosymmetric so that any coordinates with $Y<0$ are transformed via $(\boldsymbol{X}, \boldsymbol{Y}, \boldsymbol{Z}) \rightarrow(-\boldsymbol{X},-\boldsymbol{Y},-\boldsymbol{Z})$ (Matthies et al., 2005). Finally, the stereographic projection is used to produce Figure 5.4, where the polar coordinates of each point are given by $(\chi, \varphi)$, following the convention of traditional pole figure analysis (see e.g. Engler \& Randle 2010). It should be noted that this representation allows for physical sample rotations (i.e. rotations about $\boldsymbol{Y}$ ) to correspond to the angle $\varphi$, which makes it simple to determine which sample rotations will result in the best coverage of the pole figure. Figure 5.4 shows the detector coverage before texture binning (a), and after texture binning with 1 (b), 2 (c), 3 (d) and 4 (e) sample orientations $\left(\omega=0^{\circ}, 55^{\circ}, 90^{\circ}\right.$ and $\left.155^{\circ}\right)$ 


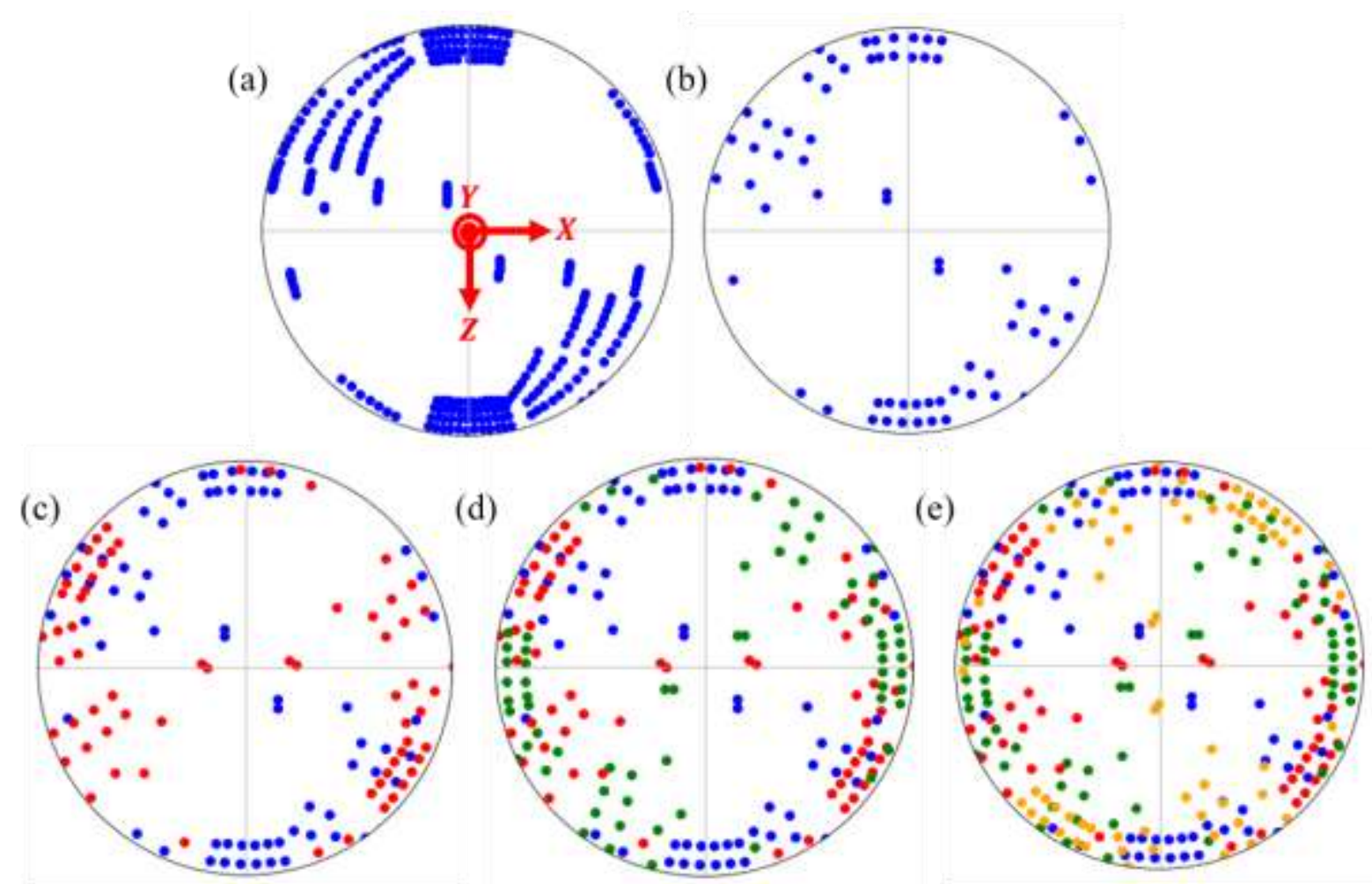

Figure 5.4 NOMAD pole figure coverage: (a) binned by detector tube, with instrument coordinates; (b) texture binning scheme (65 bins), $\omega=0^{\circ}$ (blue); (c) $\omega=0^{\circ}, 55^{\circ}$ (red); (d) $\omega=0^{\circ}, 55^{\circ}, 90^{\circ}$ (green); and (e) $\omega=0^{\circ}, 55^{\circ}$, $90^{\circ}, 155^{\circ}$ (yellow).

All coordinates described above refer to detector bins rather than individual detector pixels, and the values for each bin are determined by arithmetically averaging the coordinates for all pixels in that bin. This means that there is a certain spread about the nominal (average) value and that each detector bin represents information from a finite region of orientation space, not from a single point. Likewise, in the data reduction, the data from each detector is averaged over the entirety of the bin. In other words, the assumption is that the texture is relatively homogeneous over this small spread (never more than $\pm 3^{\circ}$ in $\chi$ or $\varphi$ ) in orientation space. For sharp textures, this averaging may be too coarse, resulting in fine features appearing artificially diffuse or failing to appear altogether. Nonetheless, the current binning strategy will be shown to work well in the present case.

\subsubsection{Data Collection}

Each specimen was placed in a $10 \mathrm{~mm}$ diameter cylindrical vanadium (V) can and secured with thin tape (safely above the location of the beam spot) to minimize motion during mounting and measurement. The beam has a circular cross-section roughly $8-10 \mathrm{~mm}$ in diameter, meaning that the 
full width of the specimen is immersed in the neutron beam during measurement. All detectors have an obstructed view of the specimen, resulting in a gauge volume of approximately $200 \mathrm{~mm}^{3}$. To preserve and record the orientation of the specimen with respect to the instrument, the can was marked to line up with the mark on the specimen and mounted to the sample stick. Measurements were performed at $\omega=0^{\circ}, 55^{\circ}, 90^{\circ}$ and $145^{\circ}$, for approximately 30 minutes each. For background, the empty V can was measured at $\omega=0^{\circ}$. As the experiment required a change of sample environment, a calibration standard (diamond powder) was measured, in addition to a $6 \mathrm{~mm}$ diameter solid $\mathrm{V}$ rod and the empty sample environment (all at $\omega=0^{\circ}$ ).

\subsubsection{Data Reduction and RITA}

The typical NOMAD data reduction strategy has been modified in Mantid (Arnold et al., 2014) to average over each detector rather than each ring and to generate the GSAS data files needed for MAUD Rietveld analysis. The 65 reduced diffraction patterns (“DP”) are corrected for background due to the sample environment and normalized with respect to incoherent scatter according to

$$
D P=\sum_{i=1}^{N}\left(\frac{\text { sample }_{i}-B G_{i}}{V_{i}-(V, B G)_{i}}\right)
$$

where the subscript $i$ represents a given TOF interval (or "bin"), $N$ is the total number of TOF intervals, and sample, $B G, V$, and $(V, B G)$ are the intensity data for the sample in the $\mathrm{V}$ (vanadium) can (the actual data), the empty V can, the V rod, and the empty sample environment, respectively. In words, the diffraction pattern is understood as a discrete set of intensity vs. TOF points, and equation 5.1 means that the subtractions and normalization are performed point-wise. The reduced diffraction pattern, then, is simply the sum of all the points after the quantity in parentheses has been computed. Though neutron scattering from $\mathrm{V}$ is almost purely incoherent, small diffraction peaks still appear and are "stripped" from the denominator before application of the formula. A Python script was written to implement the modified "SNSPowderReduction" algorithm in Mantid, and a detector grouping file, calibration file, and data files for all four quantities appearing in equation 5.1 are also needed. All of these are now included in the standard distribution of NOMAD data. The output from Mantid comes 
in the form of GSAS datafiles, which contain TOF (or optionally d-spacing), intensity and estimated standard deviation (ESD) listed in three columns for each spectrum and can be read by either GSAS or MAUD.

Spatial detector information (i.e. $r, 2 \theta, \eta$ ) is known from the design of the instrument and is therefore fixed during the texture analysis, but the precise relationship between TOF and d-spacing is established for every detector by the diffractometer constants "difc." Mantid compares the observed TOF spectra, from measurements of the calibration standard, to the known d-spacings of that material (i.e. diamond) to determine reasonable starting values for these parameters ( "calibrate" the detectors), but they are also refined during the MAUD analysis. Since they determine the relationship between TOF and d-spacing, the difc parameters define the position of the diffraction peaks. The peak shape and width is modeled by the MAUD implementation of the GSAS TOF profile function 3, as described on page 147-148 of Larson et al. 2004. Only $\sigma_{1}$ and $\sigma_{2}$ are refined for each spectrum during the analysis, as the refinement tends to become unstable if the $\alpha$ or $\beta$ parameters are not fixed. Finally, each detector bin is given one refinable scale factor parameter that determines the overall intensity scale of the corresponding spectrum.

Though the data were already calibrated within Mantid, it was determined useful to first perform a refinement on the diamond powder data, to better determine the instrumental parameters for subsequent analysis of the textured sample. Background parameters were also included and refined to ensure a reasonable fit. The refined instrumental parameters were then saved in a MAUD database for further use. Then, the data for the textured $\mathrm{Al}$ sample were imported as two separate sets so that the TOF ranges included 12 and 16 diffraction peaks for the low and high angle detectors, respectively. The total number of spectra was $65 n$, where $n$ is the number of rotations ( 4 or 3 in this case). Three background parameters $\left(2^{\text {nd }}\right.$ order polynomial) and one scale parameter were supplied for each spectrum for totals of $(65 \times 3) n=195 n$ and $65 n$, respectively. These were first refined for each of the $n$ datasets sequentially and then again at multiple later stages in the refinement process.

The OD was computed with either the E-WIMV or harmonic algorithm in MAUD (Lutterotti et al., 2004), which offers many choices of texture algorithm. The E-WIMV (direct) algorithm works 
quite well for sharper textures, whereas the harmonic (series-based) method is better for weaker textures (Engler and Randle, 2010). See Sections 1.4.2.1 and 1.4.2.2 for a more detailed description of series-based and direct methods, respectively. Both were employed in this study to explore the effects of algorithm type on the texture results. The E-WIMV algorithm was implemented with $5^{\circ}$ resolution and the harmonic with $L_{\max }=22$. Additional choices of algorithm type and resolution were attempted with less favorable agreement to the experimental data.

A more detailed procedure for texture measurement and analysis on NOMAD is given in a separate document distributed as a manual for NOMAD users.

\subsection{Results}

The orientation distribution function (ODF) was computed for each dataset via the MTEX algorithm with default resolution ( $5^{\circ}$ for all ODs in this case). Figure 5.5 shows pole figures recalculated from the ODF for the XRD and NRSF2 experimental pole figure data, in addition to the two sets of NOMAD pole figure data recalculated from the MAUD-based OD. The $R_{w p}$ values for the E-WIMV and harmonic methods were $21.0 \%$ and $21.1 \%$, respectively. Note that the OD is computed twice for the NOMAD data: once in MAUD using full RITA (called the OD), and again in MTEX for consistency with the NRSF2 and XRD results (called the ODF). To ensure consistent alignment of the pole figures, an additional algorithm available in MTEX for detecting sample symmetry was employed to determine the axes of orthotropic symmetry in the ODF and center the data accordingly. 
(a)

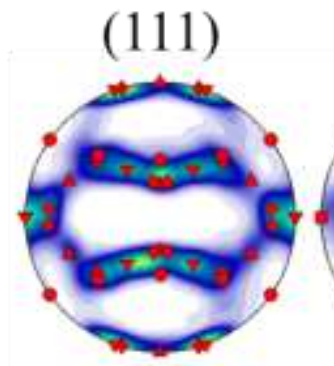

(200)

(220)

(b)
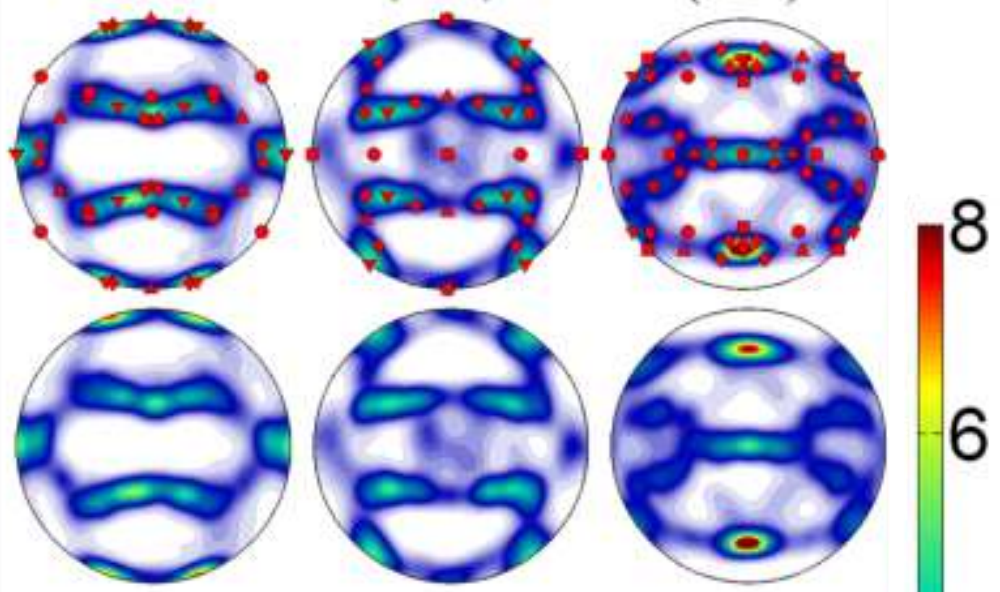

(c)
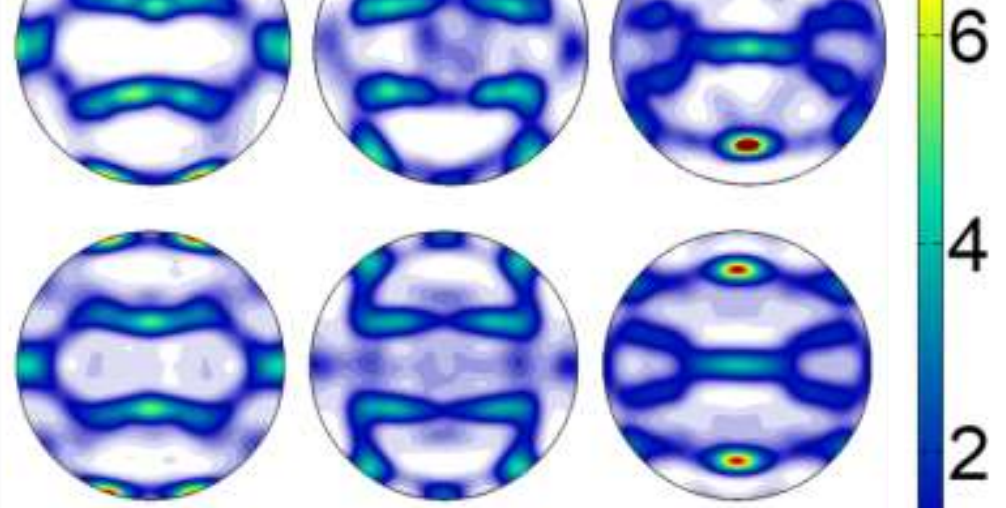

(d)
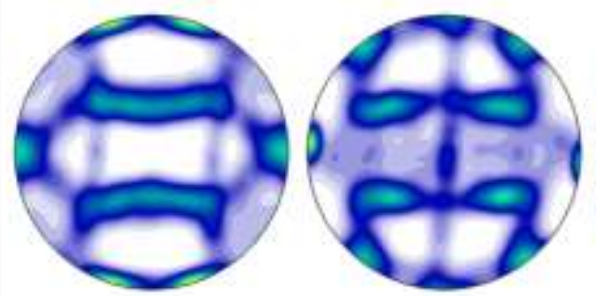

(e)
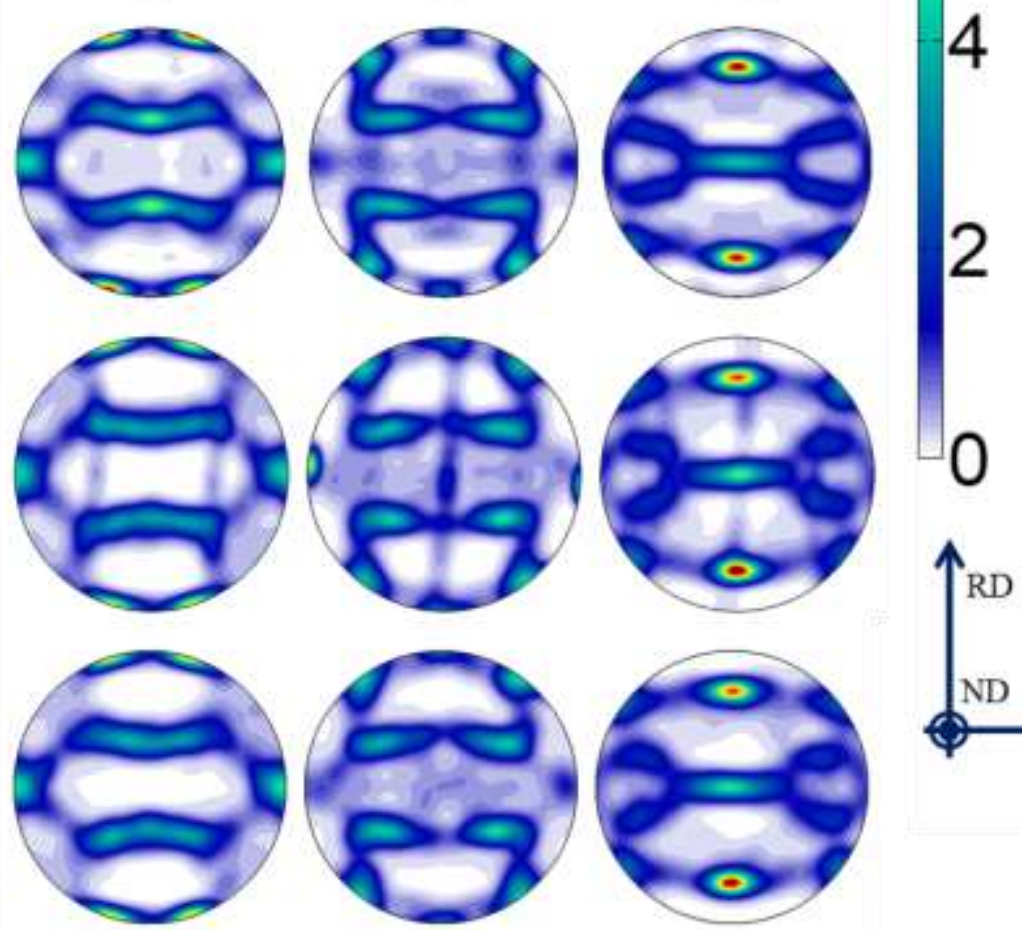

Figure 5.5 Pole figures (units: MRD) recalculated from the ODF for (a) XRD, annotated; (b) XRD; (c) NRSF2; NOMAD: (d) E-WIMV and (e) harmonic. The annotations in (a) are the FCC rolling and recrystallization "ideal" texture components copper $\boldsymbol{\Delta}$, brass $\boldsymbol{\nabla}, \mathrm{S} \boldsymbol{\nabla}$, Goss $\boldsymbol{Q}$, and cube $\square$.

After producing the plots shown in Figure 5.5, orthotropic specimen symmetry was imposed for all four of the ODFs to mitigate statistical variations due to possibly sampling grains from different locations in the plate. Figure 5.6 shows the maximum intensity near the beta fiber ideal components (copper, $\mathrm{S}$, and brass) plotted in constant $\varphi_{2}$ sections (Kocks et al., 1998) for each of the four ODFs. Note that $\varphi_{2}=45^{\circ}, 65^{\circ}$ and $90^{\circ}$ correspond to copper, $S$ and brass, respectively. 


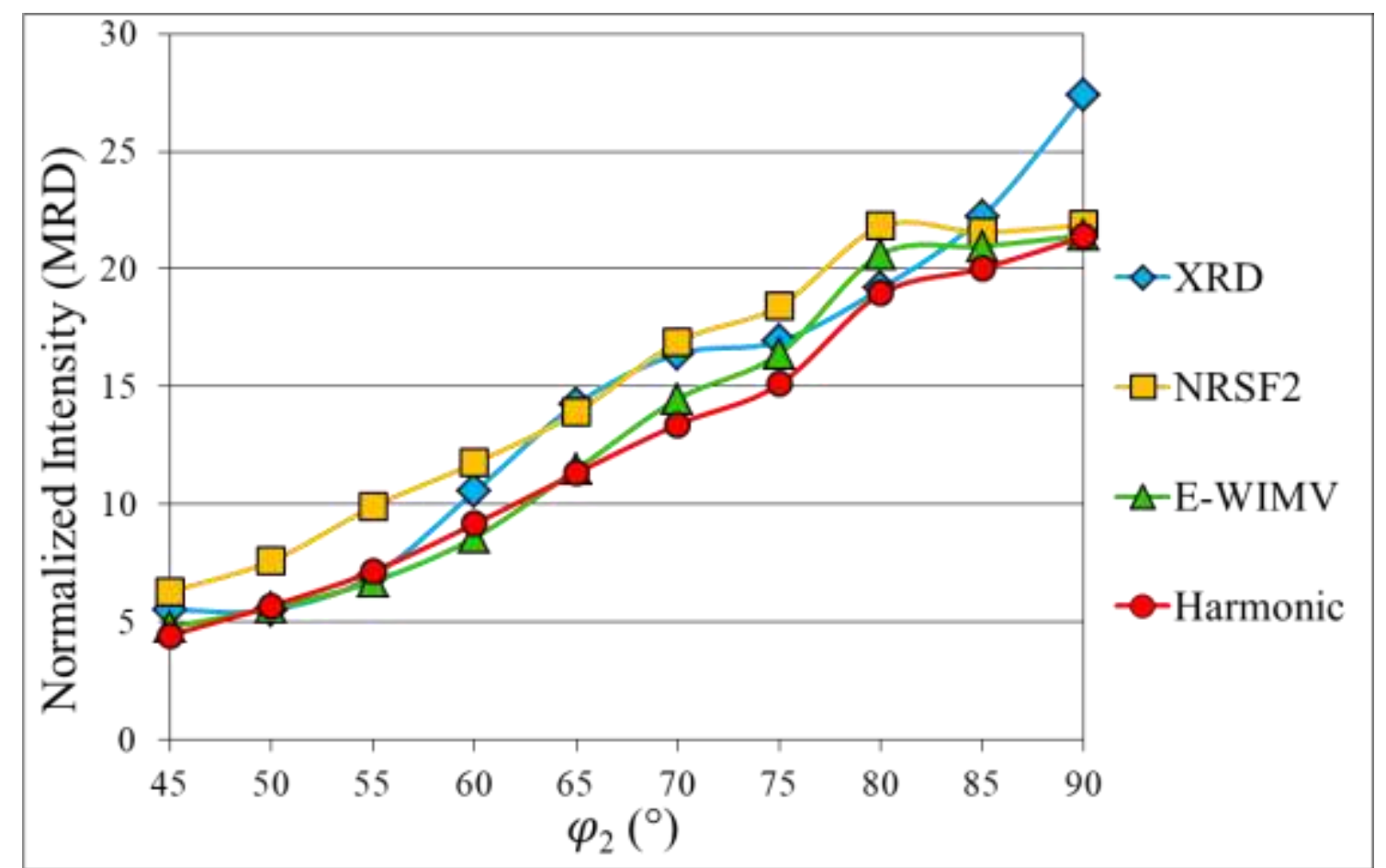

Figure 5.6 Maximum ODF intensity in constant sections along the beta fiber skeleton line. Orthotropic (point group $\mathrm{mmm}$ ) specimen symmetry is assumed.

Additionally, the texture index $J_{O D F}$ was computed, and the volume fractions of the five texture components annotated in Figure 5.5(a) were computed with $\pm 10^{\circ}$ tolerance for each of the four ODFs. These consist of the deformation components copper, brass, and S, and the recrystallization components Goss and cube, which are commonly observed in rolled and recrystallized FCC alloys (Engler and Randle, 2010). In addition, the strength of rolling (Roll) vs. recrystallization (RXX) texture components was estimated by computing the ratio ("Roll/RXX.") of the sum of the volume fractions of the Goss and cube components (RXX) to that of the copper, S and brass (Roll) components. The results are compiled in Table 5.1.

Table 5.1 Volume fraction (in \%) of several FCC rolling and recrystallization components and $J_{O D F}$ (values computed in MAUD in parentheses) for each of the four ODFs (orthotropic specimen symmetry), in addition to the XRD 3/8-plane sample ODF. The ratio "Roll/RXX" was computed by dividing the sum of the first three rows (rolling components) by the sum of rows 4 and 5 (recrystallization components).

\begin{tabular}{|c|c|c|c|c|c|c|c|}
\hline & Indices & $\begin{array}{c}\text { Bunge Euler Angles } \\
\left(\varphi_{1} \Phi \varphi_{2}\right)\end{array}$ & $\begin{array}{c}\text { XRD } \\
1 / 2\end{array}$ & $\begin{array}{c}\text { XRD } \\
3 / 8\end{array}$ & NRSF2 & E-WIMV & Harmonic \\
\hline Copper & $\{112\}<111>$ & $90^{\circ} 30^{\circ} 45^{\circ}$ & 8.2 & 13.7 & 7.2 & 8.1 & 8.2 \\
\hline S & $\{123\}<634>$ & $59^{\circ} 34^{\circ} 65^{\circ}$ & 15.1 & 12.1 & 14.0 & 11.8 & 13.2 \\
\hline Brass & $\{011\}<211>$ & $35^{\circ} 45^{\circ} 0 / 90^{\circ}$ & 32.8 & 33.5 & 29.6 & 31.0 & 30.9 \\
\hline Cube & $\{001\}<100>$ & $0^{\circ} 0^{\circ} 0 / 90^{\circ}$ & 7.2 & 3.6 & 6.2 & 8.9 & 6.8 \\
\hline Goss & $\{011\}<100>$ & $0^{\circ} 45^{\circ} 0 / 90^{\circ}$ & 1.2 & 1.4 & 8.0 & 4.8 & 4.2 \\
\hline$J_{O D F}$ & - & - & 7.2 & 7.3 & 6.5 & $6.3(7.5)$ & 5.9 \\
\hline Roll/RXX & - & - & 6.7 & 11.8 & 3.6 & 3.7 & 4.8 \\
\hline
\end{tabular}


To explore a number of effects that are discussed in detail in the ensuing section, six more ODFs were computed and analyzed. First, the ODF was computed from XRD pole figure data for a specimen sectioned at the 3/8-plane of the Al plate, roughly $5 \mathrm{~mm}$ closer to the surface than the midplane specimen. The NOMAD data were analyzed again via E-WIMV with $5^{\circ}$ resolution, using data from only three, two, and one rotational sample positions. The pole figure coverage for each of these is shown in Figure 5.4(d), (c) and (b), respectively. The NRSF2 data were re-analyzed using the singly and doubly reduced datasets, whose pole figure coverage is shown in Figure 5.2(b) and (c), respectively. The results are compared in Figure 5.7(d). For consistency with the previously presented results, orthotropic specimen symmetry was imposed for each of these six additional ODFs.
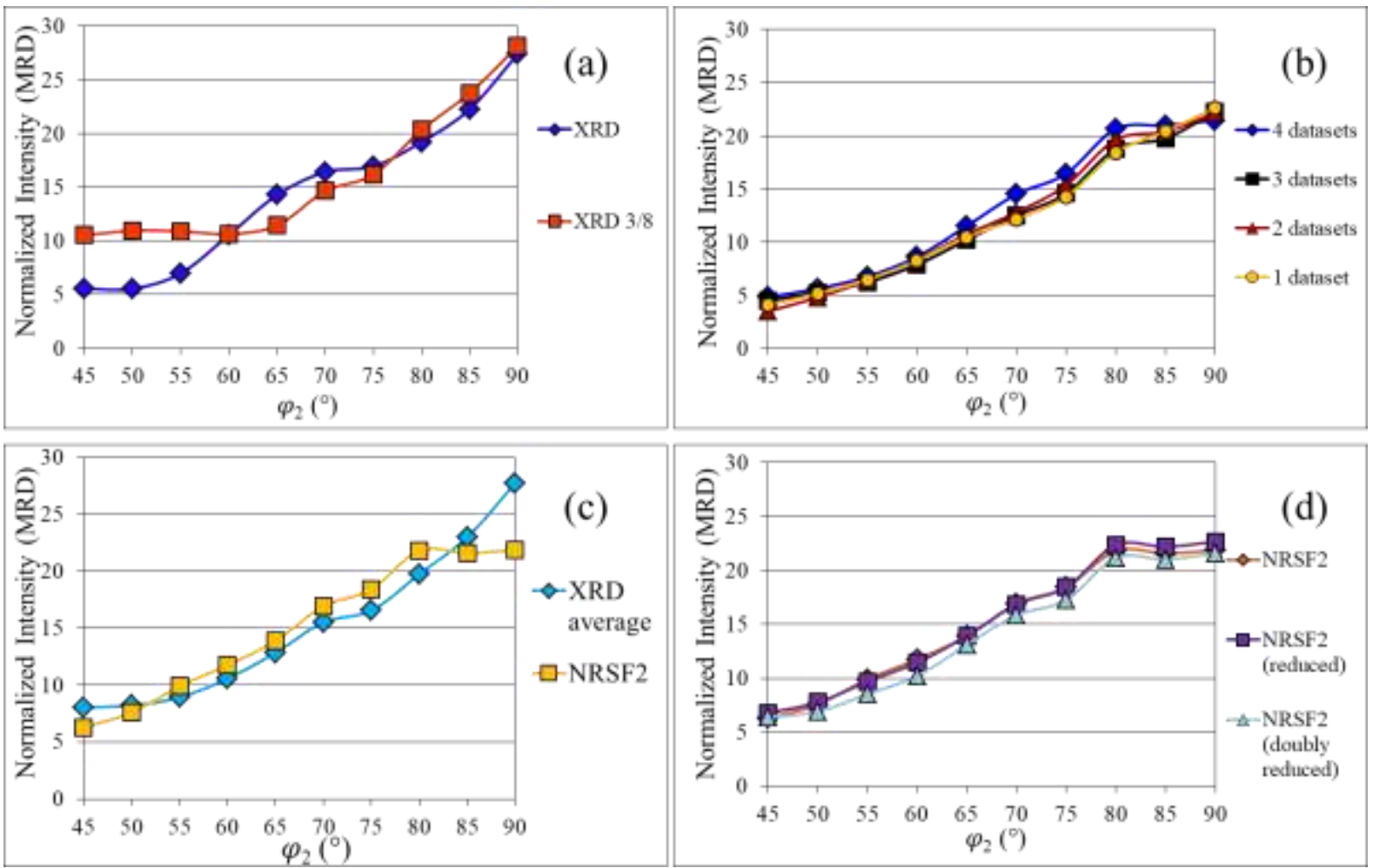

Figure 5.7 Maximum ODF (orthotropic specimen symmetry) intensity in constant sections along the beta fiber skeleton line for (a) XRD at mid-plane (original) and 3/8-plane, (b) NOMAD (E-WIMV, $5^{\circ}$ resolution) for 4, 3, 2 and 1 rotational sample positions, (c) NRSF2 and arithmetic average of XRD mid-plane and 3/8-plane and (d) NRSF2 with two possible reduction schemes.

\subsection{Discussion}

Though the results are qualitatively similar across the different methods, there are some differences in both the overall strength and the strength of various components. The XRD results 
suggest an appreciably stronger overall texture $\left(J_{O D F}\right)$, and a much smaller volume of the Goss component than any of the neutron results (Table 5.1). Note, however, that MAUD computes the largest value of $J_{O D F}$ for the E-WIMV OD, suggesting that the second ODF computation in MTEX in general results in an artificially weak estimation of the texture. This claim is corroborated by the fact that the NRSF2 results (for which the ODF was only computed once) show systematically stronger intensities along the length of the beta fiber, and in particular near the $S$ orientation $\left(\varphi_{2}=65^{\circ}\right)$. The deviation between the NRSF2 and NOMAD results is as large as $32 \%$ at $\varphi_{2}=55^{\circ}$ and remains in the 20-30\% range until $\varphi_{2}=75^{\circ}$ where the agreement is quite good (within 5\%). The XRD results fall between the two along the fiber, except near brass $\left(\varphi_{2}=90^{\circ}\right)$, where they differ from both the NRSF2 and NOMAD results by about $20 \%$. All NOMAD datasets agree quite closely, and it is particularly interesting to note that even with one dataset, the agreement is within $20 \%$ along the entire length of the beta fiber. This conclusion is corroborated by volume fraction estimates (not shown), which agree quite closely across all four of the NOMAD ODFs.

It is hypothesized that differences between the XRD and neutron results are partially due to differences in the sampled gauge volume for each method. Neutron diffraction probes a much larger volume of grains ( $30 \mathrm{~mm}^{3}$ for NRSF2, $200 \mathrm{~mm}^{3}$ for NOMAD) than does XRD $\left(\sim 3 \mathrm{~mm}^{3}\right)$, and more importantly, the probed region in this particular XRD experiment is thin along the ND because of the experimental geometry. On the other hand, the neutron diffraction information comes from a gauge volume with dimension $>3 \mathrm{~mm}$ (for NRSF2, this depends on the tilt and rotation; for NOMAD, it is constant and considerably larger than $3 \mathrm{~mm}$ ) along the ND and therefore is averaged over this entire region. Thus, it is possible that an appreciably smaller volume of grains is sampled in the XRD experiment, leading to poorer grain statistics and thus fluctuations in the texture estimation at certain components.

From Figure 5.7(a), it is clear that the location of the specimen through the thickness of the plate affects the texture as measured by XRD, particularly near the copper component $\left(\varphi_{2}=45^{\circ}\right)$ where the maxima of the mid-plane and $3 / 8$ plane data differ by over $90 \%$. This observation was corroborated by calculating the volume fraction of copper for the 3/8-plane sample: it was found to 
differ from the mid-plane sample by $67 \%$. The Roll/RXX ratio was even higher for the 3/8-plane sample (11.8), mostly due to a similarly low volume fraction of the Goss component (1.4\%) and an even lower volume fraction of the cube component (3.6\%). Though it is doubtful that any definitive conclusion can be drawn regarding the tendency for XRD to oversample deformed grains rather than recrystallized ones, it is clear that a qualitatively different texture is measured at the mid-plane vs. the 3/8-plane and that the results are still more similar to each other than to any of the neutron results.

Since the XRD datasets are of the same form (i.e. incomplete pole figures scanned on a $5^{\circ} \times$ $5^{\circ}$ grid, corrected for defocusing and background) and were analyzed in the exact same way, the difference must be attributable to either a true variation through the thickness of the plate on scales $\sim 5$ $\mathrm{mm}$ or, more likely, statistical scatter associated with sampling a non-representative population of grains. This claim is further substantiated by Figure 5.7(c), where the average of the results derived from the two XRD measurements is compared to the NRSF2 results. That such averaging improves the agreement between the XRD and NRSF2 results implies that the XRD experiment probes a statistically anomalous population of grains, whereas the NRSF2 experiment alleviates this issue by sampling many more grains. Note that this effect is likely not a cause for differences between the NRSF2 and NOMAD data, since, in the NRSF2 experiment, the number of sampled grains is already quite large (on the order of $10^{6}$ ). Thus, there is little to be gained from improving the statistics further by sampling even more grains (order $10^{7}$ ) in the NOMAD experiment. It is nonetheless reasonable to conclude that, other things (most notably the analysis procedure employed) being equal, neutron diffraction will yield the most accurate description of the bulk texture.

Since the results from both neutron diffraction experiments (i.e. on NOMAD and NRSF2) differ quite significantly, in this case there must be some inherent deviation purely due to the form of the experimental data or the analysis procedure used, rather than to differences in sampled gauge volume. As mentioned, the data from NOMAD is unavoidably (in order to utilize RITA) passed to MTEX as pole figures already recalculated from an "experimental" OD. This is in contrast to both the XRD and NRSF2 data, which are passed directly to MTEX after some corrections due to the experimental set-up but no prior ODF calculation. As demonstrated in Chapters 2 and 3, every additional ODF computation using a series-based method such as the MTEX or harmonic algorithm 
tends to smooth over sharp features in orientation space, resulting in weaker overall texture estimation. This claim is consistent with the results in Table 5.1 (most notably $J_{O D F}$ ) and Figure 5.6, where the estimated texture is predictably stronger using the XRD and NRSF2 data (ODF computed once) than using the NOMAD data (OD computed twice).

Another possible source of deviation is the difference in pole figure coverage: on NRSF2, the pole figure is covered roughly uniformly on a $6.5^{\circ} \times 5^{\circ}$ grid, whereas the XRD and NOMAD data are reported to MTEX on a $5^{\circ} \times 5^{\circ}$ grid. This causes some minor but observable differences in the experimental pole figures between the NRSF2 and XRD data (particularly near the outer regions where the coverage is sparser), so it stands to reason that it might cause differences in the ODF estimation when comparing either of the NOMAD or XRD data to the NRSF2 data. However, this factor is almost certainly less significant than the smoothing caused by multiple rounds of ODF computation (when comparing NRSF2 to NOMAD) and also likely less important than differences in sampled gauge volume (when comparing XRD to neutron diffraction). A separate but related issue is the difference between incomplete pole figures of the XRD experiment (coverage only out to $\chi=80^{\circ}$ ) and the complete pole figures (coverage out to $\chi=90^{\circ}$ ) of the NRSF2 experiment, as shown in Figure 5.2(a) and (b), respectively. This difference is known empirically to be insignificant: the NRSF2 data were truncated below $\chi=80^{\circ}$ and no noticeable effect on the results was observed.

For the reduction scheme proposed for NRSF2, the agreement between the singly reduced and original datasets is within $4 \%$ along the entire length of the fiber including $<1 \%$ near S $\left(\varphi_{2}=60^{\circ}-\right.$ $\left.70^{\circ}\right)$.The doubly reduced scheme predictably lowers the quality, though the agreement is still within $6 \%$ except near copper $\left(\varphi_{2}=45^{\circ}-55^{\circ} ; 14 \%\right.$ difference $)$. In order to compromise between minimal loss of data and improved measurement speed, a scheme between these two approaches is proposed for further measurement: three $\chi$ rings, as before, but at consecutive resolutions of $5^{\circ}, 7.5^{\circ}$ (as opposed to $5^{\circ}$ and $10^{\circ}$ in the singly and doubly reduced schemes, respectively), and $10^{\circ}$. This means that 156 (instead of 216) measurement points can be used, meaning that a full measurement can be completed in approximately 17 hours. Furthermore, that lowering the coverage of the pole figure (quite dramatically in the doubly reduced case) tends to result in very small differences again supports 
the claim that the difference in coverage of NRSF2 vs. XRD (i.e. $6.5^{\circ} \times 5^{\circ}$ vs. $5^{\circ} \times 5^{\circ}$ ) is an insignificant factor.

Now that we have identified slight deviations in the texture observed between the various methods and commented on their possible sources, we consider the importance of such differences by quantitatively assessing their effect on both texture simulation and polycrystalline property prediction. In the literature, there are a large number of studies that model the textural evolution during deformation and/or recrystallization processes and that typically use experimental data as a benchmark for their proposed simulation models (Engler, 2002; Li et al., 2004). However, the experimental texture itself can be subject to a certain level of uncertainty depending on the measurement and analysis technique used, as demonstrated in this chapter. A typical simulation result will differ from the experimental result at any point along the length of the beta fiber by about 5-15 MRD, depending on the rolling reduction (Engler, 2002; Li et al., 2004; Van Houtte et al., 2005). Depending on the specific alloy, rolling reduction and other factors, differences are typically most notable near the S component $\left(\varphi_{2} \sim 60^{\circ}-70^{\circ}\right)$; where a 5 MRD deviation would be $50 \%$ (e.g. 10 MRD experimental vs. 15 MRD predicted). At the same time, if (say) NRSF2 were used to measure the texture instead of NOMAD (along with associated differences in analysis procedure), the "uncertainty" in the experimental texture, purely based on the choice of method, could be around 30\% ( $\sim 27 \%$ difference at $\varphi_{2}=60^{\circ}$, Figure 5.6). Therefore, it is sensible to interpret experimental texture results with a certain degree of caution and to measure and analyze them with a number of different methods whenever possible. For the most accurate bulk texture measurements, neutron diffraction is recommended due to its superiority in grain statistics. The choice of OD computation algorithm depends on the strength of the texture: for weaker textures, the harmonic method is adequate (though, still, a high $L_{\max }$ should be chosen to limit truncation error), but for stronger textures a direct method such as E-WIMV is recommended.

An alternative approach to assessing the importance of the observed differences is to directly explore their effect on polycrystalline tensor properties. For properties such as elastic stiffness $C_{i j k l}$, the polycrystalline tensor $C_{\text {poly }}$ can be computed from the single crystal tensor $C_{\text {single }}$ and the ODF 
$f(g)$. It stands to reason that if the single crystal is essentially isotropic, the polycrystal will also be, regardless of the texture. For this reason, it is prudent to use $C_{\text {single }}$ from a strongly anisotropic crystal such as $\beta$-brass $(\mathrm{CsCl}$ or $\mathrm{B} 2$ structured) to most dramatically display the effect that differences in $f(g)$ might have on $C_{\text {poly }}$. Anisotropy of the stiffness is typically reported by either the coefficients of $C_{i j k l}$ or more concisely by the so-called Zener parameter, which is given in the Voigt notation by $A=\frac{2 C_{44}}{C_{11}-C_{12}}$ and approaches unity for perfect isotropy (Zener, 1948). Table 5.2 shows $A$ values and the 9 relevant coefficients of the $C_{\text {poly }}$ matrix, computed from each of the five ODFs using the single crystal properties of $\beta$-brass (Simmons and Wang, 1971) and the Hill averaging method (Hill, 1952). The Hill method involves taking the arithmetic average of the Voigt and Reuss approximations for the polycrystalline elasticity tensor, which are based on "orientation averaging" of the single crystal stiffness $\left(C_{\text {single }}\right)$ and compliance $\left(S_{\text {single }}=C_{\text {single }}^{-1}\right)$ tensors, respectively, based on the ODF $f(g)$ (Man and Huang, 2011).

Table 5.2 Elastic anisotropy of $\beta$-brass based on the four ODFs.

\begin{tabular}{|c|c|c|c|c|c|}
\cline { 2 - 6 } \multicolumn{1}{c|}{} & $\begin{array}{c}\beta \text {-brass } \\
\text { single crystal }\end{array}$ & XRD & NRSF2 & E-WIMV 5 & Harmonic \\
\hline $\mathrm{A}$ & 18.684 & 0.752 & 0.753 & 0.779 & 0.760 \\
\hline $\mathrm{C}_{11}$ & 52 & 134 & 135 & 130 & 134 \\
\hline $\mathrm{C}_{12}$ & 34 & -18 & -16 & -14 & -16 \\
\hline $\mathrm{C}_{13}$ & 34 & 3 & 0 & 3 & 1 \\
\hline $\mathrm{C}_{22}$ & 52 & 149 & 147 & 146 & 147 \\
\hline $\mathrm{C}_{23}$ & 34 & -12 & -12 & -12 & -12 \\
\hline $\mathrm{C}_{33}$ & 52 & 128 & 131 & 128 & 130 \\
\hline $\mathrm{C}_{44}$ & 173 & 57 & 57 & 56 & 57 \\
\hline $\mathrm{C}_{55}$ & 173 & 70 & 67 & 71 & 68 \\
\hline $\mathrm{C}_{66}$ & 173 & 52 & 54 & 55 & 54 \\
\hline
\end{tabular}

The largest deviation in $A$ is between the XRD and E-WIMV results but is only $4 \%$. In addition, none of the individual $C_{\text {poly }}$ components differ appreciably or systematically across the various methods. Thus, it is evident that the differences in texture discussed previously will have little effect on the elastic response of a polycrystalline aggregate, even if the single crystal is highly anisotropic. In this case, this is partly due to the moderate strength of the texture. This is encouraging 
for the study of texture-property relationships in systems with similarly weak texture or weakly anisotropic single crystals: even for a case of strong single crystal anisotropy, the polycrystalline tensor property prediction is only affected slightly in spite of seemingly substantial deviations in the texture.

\subsection{Future Work}

\subsubsection{NRSF2}

In addition to the reduced measurement schemes proposed here, which would decrease the time required for collection by about 7 hours but maintain the reliability of measurements (Figure 5.7(d)), installation of a large 2-D area detector has been proposed. The dimensions of the detector will be roughly $30 \mathrm{~cm} \times 30 \mathrm{~cm}$, which, at a sample-detector distance of $\sim 1 \mathrm{~m}$, will cover more than $15^{\circ}$ in both $\chi$ and $\varphi$. The current detector covers roughly $30^{\circ}$ in $\chi$ and $0^{\circ}$ (a vertical line detector, for purposes of texture measurement) in $\varphi$ for $6 \times 24=144$ collection points. This is exactly the same savings as the second modification proposed earlier, but it would not compromise at all on reliability. In addition, this would be advantageous for sharper textures since this reduced measurement scheme can be used with the orientation space resolution limited only by the pixel size and the data processing software. Accounting for such software limits, the effective pixel size could be as large as $35 \mathrm{~mm} \times$ $35 \mathrm{~mm}$ for $1^{\circ}$ resolution in both angles, whereas Yamagishi et al. (2011) reported resolution under 2 $\mathrm{mm}$ for a $2-\mathrm{D}{ }^{3} \mathrm{He}$ neutron detector. Even $8 \mathrm{~mm}$ pixels would provide better than $0.5^{\circ}$ resolution on NRSF2, which is adequate even for extremely sharp textures.

If detection efficiency is improved from the current arrangement, counting time can be reduced, and thus measurements can start to approach $\sim 3$ hours $(144 \times 75 \mathrm{~s}=3 \mathrm{hr})$ per pole figure. Further reductions can be made with some a priori knowledge of the location of strong fibers in orientation space, allowing for less required sample rotations. Moreover, delays due to goniometer motion and data readout could be mitigating by implementing a continuous detection approach, as is commonly used with laboratory X-ray diffractometers. These improvements, along with the potential 
to combine high-resolution residual strain (NRSF's principal use) and texture analysis in one measurement, make NRSF2 a competitive candidate for future users at ORNL.

\subsubsection{NOMAD}

Based on the results presented previously, it is possible on NOMAD to measure only one rotation on and still produce reliable results for this texture. This means that a full measurement can be completed in about 30 minutes. However, for considerably weaker or less symmetric textures (in e.g. minerals) may require additional coverage to produce reliable results. It is possible to improve the coverage either by adding additional neutron detectors or by adding a motor to allow for rotations out of the plane. Both will be explored in what follows.

Though it is likely impractical to fill out all 99 panels, it is possible to improve the coverage by populating only a few of the presently empty ones. In particular, some of the panels in the range of $2 \theta \sim 20-40^{\circ}$ fall near the center of the pole figure, which is poorly covered under the current scheme (without rotation). The improvement of the coverage is demonstrated in Figure 5.8. Moreover, these panels fall in a $2 \theta$ range that is known (from the experiments reported on in this chapter) to produce reasonable quality data, in contrast to the very high $\left(>120^{\circ}\right)$ and low $\left(<20^{\circ}\right)$ angle detectors. If the results of measurements based on the scheme demonstrated in Figure 5.8(d) are similar to ones employing the current strategy, then the number of rotations can be decreased to only two, and the measurement time reduced to $\sim 1$ hour. This, of course, is only helpful for weaker textures or for lower symmetry crystal systems, since reliable results can be produced using only one rotation for the presently studied texture Figure 5.7(b). In addition, it is likely that 30 minutes per rotation, as was employed in this study, is only necessary for excellent diffraction counting statistics, which are typically not needed for successful full-pattern analysis using RITA. 


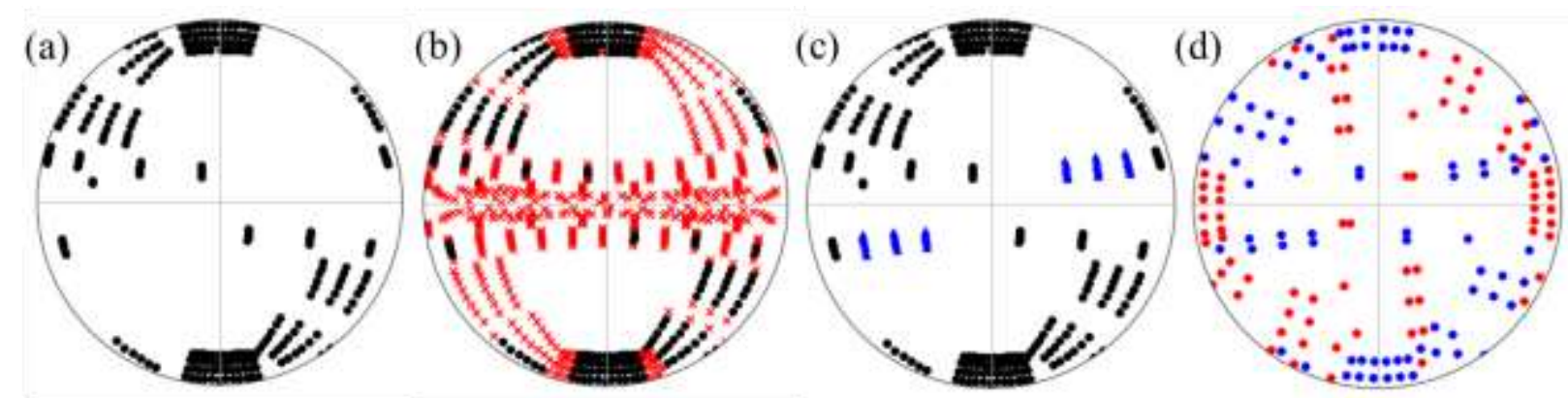

Figure 5.8 NOMAD detector coverage: (a) Current (b) Empty panels (red) included (c) Proposed additions included. (d) Binned, 0 and $90^{\circ}$ (red) with proposed additions included.

Unfortunately, adding neutron detectors can be expensive and time-consuming, so one alternative is to install a motor to enable rotations both in and out of the current plane of rotation. This will allow for greater flexibility in manipulating the sample in order to improve the coverage in general or to cover specific regions of orientation space (e.g. near a strong texture component). In addition, hopefully the installation of such a motor will be accompanied by new a sample environment that is better suited for texture measurement. The highest priorities for such a device are precise automated sample manipulation and large size to accommodate larger or oddly-shaped samples. An automated sample changing device with the ability to accommodate several samples would also be highly desirable for high-throughput texture measurements.

\subsection{Conclusion}

In this study, the capability to make efficient and reliable texture measurements on the neutron diffractometers NRSF2 and NOMAD at the ORNL is demonstrated. The data from NOMAD was processed using two different RITA algorithms in MAUD. The three datasets, (XRD, NRSF2, and NOMAD) were compared using several standard graphical and quantitative metrics. Reasonable agreement was found in each case, though some differences in overall estimated texture strength and the relative strength of some components were present due to (unavoidably) using different techniques to measure and analyze the data. The three ODFs derived from pole figure inversion (XRD and NRSF2) agree quite well, aside from differences that can be attributed to the smaller gauge volume of the XRD measurements (since the RXX components are stochastic, they tend to be less well represented in the XRD data). Given that the same measurement and analysis strategies are employed (3 measured pole figures analyzed with pole figure inversion in MTEX), the higher grain counting 
statistics of the NRSF2 measurement engender greater confidence in that measurement. The current study has also shown that the efficiency of the NRSF2 measurement can be improved by about 1/3 without loss of quality and further improvements were discussed in the context of a proposed detector upgrade.

Regarding the data obtained on NRSF2 and NOMAD, much of the difference can be attributed to computing the ODF twice (once in MAUD and again in MTEX), which artificially smooths the data but was required to put the volume fraction calculations on equal footing (all using MTEX). As evidence of this fact, it is noted that the $J_{O D F}$ values decreased from $\sim 7.5$, which agrees very well with those computed for the NRSF2 data, down to about 6.2 after the smoothing enforced by MTEX. For the present texture, it appears that a single sample position provides sufficient coverage of orientation space to accurately assess the texture, enabling a complete measurement in 30 minutes. Strategies to further improve the efficiency were provided in Section 5.6.2.

The four ODFs were also compared to texture simulation results in the literature and to each other based on their effect on material property prediction. It was found that the deviations observed between the four ODFs caused little difference in the predicted polycrystalline properties, even for a strongly anisotropic single crystal. On the other hand, comparing texture simulation results to an experimental "benchmark" should be approached with caution, as results derived from even the same experiment can vary significantly purely based on the analysis strategy employed.

\subsection{Acknowledgments}

A portion of this research used resources at the High Flux Isotope Reactor and Spallation Neutron Source, DOE Office of Science User Facilities operated by the Oak Ridge National Laboratory. This material is based upon work supported by the U.S. Department of Energy, Office of Science, Office of Basic Energy Sciences, under contract number DE-AC05-00OR22725. A tremendous debt of gratitude is owed to the following people for their helpful advice and work towards this publication: Dr. Andrew Payzant, Dr. Peter Peterson, Dr. Sven Vogel, Dr. Luca Lutterotti, Narendran Raghavan, Dr. Juan Gómez Barreiro, Dr. Lawrence Anovitz, Dr. Alexandru 
Stoica, Dr. Katharine Page, and Dr. Joerg Neuefeind. Additional special thanks is afforded to Michelle Everett and Dr. Marshall McDonnell for their tireless dedication to and invaluable help with experiments on NOMAD. Funding for much of this work is provided jointly by the Oak Ridge National Laboratory and the University of Virginia, as part of the GO! Program.

This manuscript has been authored by UT-Battelle, LLC under Contract No. DE-AC0500OR22725 with the U.S. Department of Energy. The United States Government retains and the publisher, by accepting the article for publication, acknowledges that the United States Government retains a non-exclusive, paid-up, irrevocable, worldwide license to publish or reproduce the published form of this manuscript, or allow others to do so, for United States Government purposes. The Department of Energy will provide public access to these results of federally sponsored research in accordance with the DOE Public Access Plan (http://energy.gov/downloads/doe-public-accessplan). 


\section{Conclusions and Outlook}

\subsection{Summary}

Throughout this thesis, various methods for texture measurement and analysis were explored and compared. Chapter 1 motivated the study of texture and provided the context and theoretical framework needed to understand and appreciate both conventional and modern methods for quantitative texture analysis. The pole figure inversion technique using XRD and MTEX was employed in Chapter 2 to investigate how the specific measurement and analysis technique used affects the quality of results. The proposed procedure for reducing the measurement time was shown to yield reliable results, but only if the results from both methods are analyzed in a consistent manner. The strong influence on the results of using different ODF computation parameters in MTEX (especially the kernel half-width) was demonstrated in dramatic fashion.

Chapter 3 showed how synchrotron XRD can be used to very efficiently measure the textural evolution during compression testing of a Mg-based alloy with LPSO phase by exploiting radial symmetry. RITA was implemented using MAUD, and the analysis procedure was outlined in detail. The initial textures of both the $\alpha-\mathrm{Mg}$ matrix and LPSO phases were found to be much stronger than previously reported and the differences attributed to the different analysis procedures used. The textural evolutions of both phases were also discussed in the context of the deformation behavior of the alloy. It was postulated that accommodation of the deformation in the $\alpha-\mathrm{Mg}$ phase is dominated by twinning, whereas the LPSO phase underwent substantial plastic and elastic deformation and possibly some twinning.

The texture of $\alpha$-uranium foils, as measured using both XRD and TOF neutron diffraction and analyzed with MTEX (pole figure inversion) and MAUD (RITA), respectively, was studied in chapter 4. It was demonstrated that the surface texture, as probed by XRD, differed slightly from the bulk texture measured by neutron diffraction. The pole figure inversion method, which is not often applied for studying the texture of $\alpha$-uranium because of peak overlap, was proven to work well using the outlined procedure. 
The capability to measure texture on two neutron diffractometers at the Oak Ridge National Laboratory was demonstrated in Chapter 5. The results using a variety of RITA approaches applied to multi-detector, time-of-flight (TOF) neutron diffraction measurements were compared to results from pole figure inversion methods using angular-dispersive diffraction data (both XRD and neutron diffraction). The results were found to be mostly consistent, and neutron diffraction was shown to produce the most accurate representation of the bulk texture. The differences between methods were, again, mostly attributed to differences in the chosen analysis procedures.

\subsection{Future Work}

At this point, the reader has hopefully developed an understanding of and appreciation for the numerous measurement and analysis techniques available to the modern texture analyst. Throughout this thesis, ways to improve measurement procedures have been identified and will be summarized in this section. In chapter 5, suggestions for improving the data collection speed, without compromising on the reliability of data, on both NRSF2 and NOMAD on NRSF2 were proposed. In particular, installation of a $30 \mathrm{~cm} \times 30 \mathrm{~cm}$ 2-D detector at NRSF2 will slightly improve collection times and greatly improve the capability to measure exceedingly sharp textures reliably and efficiently. Installation of additional detectors at NOMAD will have a less dramatic effect but will generally result in more reliable texture data by improving the coverage of orientation space. An alternative strategy is to install a motor to perform sample manipulations both in-plane and out-of-plane.

Another important consideration on both instruments is collection time per sample position, an issue that was not explored in detail in this thesis. To that end, it is recommended that a study be conducted in order to determine the minimum amount of time needed to collect diffraction data of sufficient quality for texture analysis on both NRSF2 and NOMAD. This will likely lead to substantially faster measurements, particularly on NOMAD where the presently employed collection time is almost certainly excessive. 


\section{References}

Aluminum Foil: Chemical and Physical Properties [WWW Document], n.d. . AJ Oster Foils, Inc. URL http://www.ajoster.com/sites/default/files/downloads/Aluminum-Foil-Data-Sheet-AJOster.pdf

Anderson, R., Carpenter, D., Kollie, T., Smith, D., 1982. Texture-property relationships in the uranium-2.4 weight percent niobium alloy, in: Metallurgical Technology of Uranium and Uranium Alloys. American Society for Metals, Metals Park, OH, pp. 231-249.

Ángeles, G., Martín-Sedeño, M., Leon-Reina, L., Compana, J.M., Aranda, M., G., A., n.d. Synchrotron X-ray Diffraction in Mineralogy and Materials Chemistry. Possibilities and Applications. Univ. Basqu. Ctry. 29-59.

Arnold, O., Bilheux, J.C., Borreguero, J.M., Buts, A., Campbell, S.I., Chapon, L., Doucet, M., Draper, N., Ferraz Leal, R., Gigg, M.A., Lynch, V.E., Markvardsen, A., Mikkelson, D.J., Mikkelson, R.L., Miller, R., Palmen, K., Parker, P., Passos, G., Perring, T.G., Peterson, P.F., Ren, S., Reuter, M.A., Savici, A.T., Taylor, J.W., Taylor, R.J., Tolchenov, R., Zhou, W., Zikovsky, J., 2014. Mantid - Data analysis and visualization package for neutron scattering and ?? SR experiments. Nucl. Instruments Methods Phys. Res. Sect. A Accel. Spectrometers, Detect. Assoc. Equip. 764, 156-166. doi:10.1016/j.nima.2014.07.029

Bachmann, F., Hielscher, R., Schaeben, H., 2010. Texture Analysis with MTEX - Free and Open Source Software Toolbox. Solid State Phenom. 160, 63-68. doi:10.4028/www.scientific.net/SSP.160.63

Bearden, J.A., 1967. X-Ray Wavelengths. Rev. Mod. Phys. 39, 78-124.

Bhattacharyya, J.J., Agnew, S.R., Lee, M.M., Whittington, W.R., El Kadiri, H., 2017. Measuring and modeling the anisotropic, high strain rate deformation of $\mathrm{Al}$ alloy, 7085, plate in $\mathrm{T} 711$ temper. Int. J. Plast. 93, 46-63. doi:10.1016/j.ijplas.2017.03.001

Bradley, E., 2013. Non-HEU Production Technologies for Molybdenum-99 and Technetium-99m. IAEA Nuclear Energy Series.

Brokmeier, H.G., Gan, W.M., Randau, C., Völler, M., Rebelo-Kornmeier, J., Hofmann, M., 2011. Texture analysis at neutron diffractometer STRESS-SPEC. Nucl. Instruments Methods Phys. Res. Sect. A Accel. Spectrometers, Detect. Assoc. Equip. 642, 87-92. doi:10.1016/j.nima.2011.04.008

Brown, D.W., Bourke, M.A.M., Clausen, B., Korzekwa, D.R., Korzekwa, R.C., McCabe, R.J., Sisneros, T.A., Teter, D.F., 2009. Temperature and direction dependence of internal strain and texture evolution during deformation of uranium. Mater. Sci. Eng. A 512, 67-75. doi:10.1016/j.msea.2009.02.004

Budai, J.D., Yang, W., Tamura, N., Chung, J.-S., Tischler, J.Z., Larson, B.C., Ice, G.E., Park, C., Norton, D.P., 2003. X-ray microdiffraction study of growth modes and crystallographic tilts in oxide films on metal substrates. Nat. Mater. 2, 487-492. doi:10.1038/nmat916

Bunge, H.J., 1989. Advantages of Neutron Diffraction in Texture Analysis. Textures Microstruct. 10, 265-307. doi:10.1155/TSM.10.265

Bunge, H.J., 1982. Texture Analysis in Materials Science. Butterworth, London.

Bunge, H.J., 1965. Zur Darstellung allgemeiner Texturen. Int. J. Mater. Res. 56, 872-874.

Bunn, J.R., Penumadu, D., Lou, X., Hubbard, C.R., 2014. Effect of multi-axial loading on residual strain tensor for 12L14 steel alloy. Metall. Mater. Trans. A Phys. Metall. Mater. Sci. 45, 38063813. doi:10.1007/s11661-014-2355-5

Burke, J.E., Turkalo, A.M., 1957. The growth of Uranium upon thermal cycling. Trans. ASM 50, 
943-953.

Caglioti, G., Paoletti, A., Ricci, F.P., 1958. Choice of collimators for a crystal spectrometer for neutron diffraction. Nucl. Instruments 3, 223-228. doi:10.1016/0369-643X(58)90029-X

Cahn, R.W., 1953. Plastic Deformation of Alpha-Uranium; Twinning and Slip. Acta Metall. 1, 49-70. doi:10.1016/0001-6160(53)90009-1

Calhoun, C.A., Garlea, E., Mulay, R.P., Sisneros, T.A., Agnew, S.R., 2015. Investigation of the effect of thermal residual stresses on deformation of alpha-uranium through neutron diffraction measurements and crystal plasticity modeling. Acta Mater. 85, 168-179. doi:10.1016/j.actamat.2014.11.007

Choi, C.S., Staker, M., 1996. Neutron diffraction texture study of deformed uranium plates. J. Mater. Sci. 31, 3397-3402.

Dimasi, E., Sarikaya, M., 2004. Synchrotron X-ray microbeam diffraction from abalone shell. J. Mater. Res. 19, 1471-1476.

Du, A., Wan, C., Qu, Z., Wu, R., Pan, W., 2010. Effects of texture on the thermal conductivity of the LaPO4 monazite. J. Am. Ceram. Soc. 93, 2822-2827. doi:10.1111/j.1551-2916.2010.03779.x

E112-13, ASTM Standard, Standard Test Methods for Determining Average Grain Size, 2013. . Materials Park, $\mathrm{OH}$.

Eichhorn, R.M., 1965. Automatic Pole Figure Plotter. Rev. Sci. Instrum. 36, 997-1000.

Engler, O., 2002. A new approach to more realistic rolling texture simulation. Adv. Eng. Mater. 4, 181-186. doi:10.1002/1527-2648(200204)4:4<181::AID-ADEM181>3.0.CO;2-3

Engler, O., 1999. Influence of Number and Arrangement of Single Orientation Measurements on their Statistical Relevance, in: Proceedings of the Twelfth International Conference on Textures of Materials. Montreal, Canada, pp. 68-73.

Engler, O., Randle, V., 2010. Introduction to Texture Analysis; Macrotexture, Microtexture, and Orientation Mapping. CRC Press, Taylor \& Francis Group, Boca Raton, FL.

Foote, F., 1956. Physical metallurgy of uranium, in: Progress in Nuclear Energy Series V. McGrawHill, New York, pp. 81-201.

Fultz, B., Howe, J., 2008. Mass attenuation coefficients for characteristic K $\alpha$ X-rays, in: Transmission Electron Microscopy and Diffractometry of Materials. Springer, p. 692.

Garces, G., Morris, D.G., Muñoz-Morris, M.A., Perez, P., Tolnai, D., Mendis, C., Stark, A., Lim, H.K., Kim, S., Shell, N., Adeva, P., 2015a. Plasticity analysis by synchrotron radiation in a Mg97Y2Zn1 alloy with bimodal grain structure and containing LPSO phase. Acta Mater. 94, 78-86. doi:10.1016/j.actamat.2015.04.048

Garces, G., Muñoz-Morris, M.A., Morris, D.G., Jimenez, J.A., Perez, P., Adeva, P., 2014. The role of extrusion texture on strength and its anisotropy in a Mg-base alloy composed of the LongPeriod-Structural-Order phase. Intermetallics 55, 167-176. doi:10.1016/j.intermet.2014.07.015

Garces, G., Perez, P., Cabeza, S., Lin, H.K., Kim, S., Gan, W., Adeva, P., 2015b. Reverse tension/compression asymmetry of a Mg-Y-Zn alloys containing LPSO phases. Mater. Sci. Eng. A 647, 287-293. doi:10.1016/j.msea.2015.09.003

Garcés, G., Requena, G., Tolnai, D., Pérez, P., Adeva, P., Stark, A., Schell, N., 2014. Influence of rare-earth addition on the long-period stacking ordered phase in cast Mg-Y-Zn alloys. J. Mater. Sci. 49, 2714-2722. doi:10.1007/s10853-013-7967-4

Ghosh, P., Chromik, R.R., Vashegi, B., Knight, A.M., 2014. Effect of crystallographic texture on the bulk magnetic properties of non-oriented electrical steels. J. Magn. Magn. Mater. 365, 14-22. doi:10.1016/j.jmmm.2014.04.051

Gómez Barreiro, J., Wenk, H.R., Vogel, S., 2015. Texture and elastic anisotropy of a mylonitic 
anorthosite from the Morin Shear Zone (Quebec, Canada). J. Struct. Geol. 71, 100-111. doi:10.1016/j.jsg.2014.07.021

Govindarajan, S.G., Graybill, B.S., Makarewicz, P.F., Xie, Z., Solbrekken, G.L., 2013. Assembly and irradiation modeling of residual stresses in low-enriched uranium foil-based annular targets for molybdenum-99 production. Sci. Technol. Nucl. Install. 2013. doi:10.1155/2013/673535

Hagihara, K., Klnoshita, A., Sugino, Y., Yamasaki, M., Kawamura, Y., Yasuda, H.Y., Umakoshi, Y., 2010a. Plastic deformation behavior of Mg97Zn1 Y2 extruded alloys. Trans. Nonferrous Met. Soc. China (English Ed. 20, 1259-1268. doi:10.1016/S1003-6326(09)60288-0

Hagihara, K., Yokotani, N., Umakoshi, Y., 2010b. Plastic deformation behavior of Mg12YZn with 18R long-period stacking ordered structure. Intermetallics 18, 267-276. doi:10.1016/j.intermet.2009.07.014

Helstroffer, A., Villechaise, P., Gadaud, P., Andrieu, S., 2016. Effect of crystallographic texture on elastic properties of Ti-5553 $\beta$-metastable titanium alloy. Proc. 13th World Conf. Titan. 877884.

Hempel, N., Bunn, J.R., Nitschke-pagel, T., 2016. Residual stress analysis in girth-welded ferritic and austenitic steel pipes using neutron and X-ray diffraction. Residual Stress. 2016 ICRS-10 2, 229-234. doi:10.21741/9781945291173-39

Heydt, P., Luo, C.Y., Clarke, D.R., 2001. Crystallographic texture and thermal conductivity of zirconia thermal barrier coatings deposited on different substrates. J. Am. Ceram. Soc. 84, 15391544.

Hielscher, R., Schaeben, H., 2008. A novel pole figure inversion method: Specification of the MTEX algorithm. J. Appl. Crystallogr. 41, 1024-1037. doi:10.1107/S0021889808030112

Hill, R.W., 1952. The elastic behavior of a crystalline aggregate. Proc. Phys. Soc. London A, 349354.

Hirano, M., Yamasaki, M., Hagihara, K., Higashida, K., Kawamura, Y., 2010. Effect of Extrusion Parameters on Mechanical Properties of Mg97Zn1Y2 Alloys at Room and Elevated Temperatures. Mater. Trans. 51, 1640-1647. doi:10.2320/matertrans.MAW201026

Hutchings, M.T., Withers, P.J., Holden, T.M., Lorentzen, T., 2005. Introduction to the Characterization of Residual Stress by Neutron Diffraction. CRC Press, Taylor \& Francis Group, Boca Raton, FL.

Ischia, G., Wenk, H.R., Lutterotti, L., Berberich, F., 2005. Quantitative Rietveld texture analysis of zirconium from single synchrotron diffraction images. J. Appl. Crystallogr. 38, 377-380. doi:10.1107/S0021889805006059

Ivankina, T.I., Matthies, S., 2015. On the development of the quantitative texture analysis and its application in solving problems of the Earth sciences. Phys. Part. Nucl. 46, 366-423. doi:10.1134/S1063779615030077

Kawamura, Y., Hayashi, K., Inoue, A., Masumoto, T., 2001. Rapidly Solidified Powder Metallurgy Mg97Zn1Y2Alloys with Excellent Tensile Yield Strength Above 600 MPa. Mater Trans. doi:10.2320/matertrans.42.1172

Kim, D.-K., Hwang, J.-H., Kim, E.-Y., Heo, Y.-U., Woo, W., Choi, S.-H., 2017. Evaluation of the stress-strain relationship of constituent phases in AlSi10Mg alloy produced by selective laser melting using crystal plasticity FEM. J. Alloys Compd. 714, 687-697.

doi:10.1016/j.jallcom.2017.04.264

Knezevic, M., Capolungo, L., Tomé, C.N., Lebensohn, R.A., Alexander, D.J., Mihaila, B., McCabe, R.J., 2012. Anisotropic stress-strain response and microstructure evolution of textured alphauranium. Acta Mater. 60, 702-715. doi:10.1016/j.actamat.2011.10.041

Knezevic, M., McCabe, R.J., Lebensohn, R.A., Tomé, C.N., Liu, C., Lovato, M.L., Mihaila, B., 
2013a. Integration of self-consistent polycrystal plasticity with hardening law based on dislocation densities in finite-elements: application to low-symmetry metals. J. Mech. Phys. Solids 61, 2034-2046.

Knezevic, M., McCabe, R.J., Tomé, C.N., Lebensohn, R.A., Chen, S.R., Cady, C.M., Gray, G.T., Mihaila, B., 2013b. Modeling mechanical response and texture evolution of alpha-uranium as a function of strain rate and temperature using polycrystal plasticity. Int. J. Plast. 43, 70-84. doi:10.1016/j.ijplas.2012.10.011

Kockelmann, W., Chapon, L.C., Radaelli, P.G., 2006a. Neutron texture analysis on GEM at ISIS. Phys. B Condens. Matter 385-386 I, 639-643. doi:10.1016/j.physb.2006.06.091

Kockelmann, W., Siano, S., Bartoli, L., Visser, D., Hallebeek, P., Traum, R., Linke, R., Schreiner, M., Kirfel, A., 2006b. Applications of TOF neutron diffraction in archaeometry. Appl. Phys. A Mater. Sci. Process. 83, 175-182. doi:10.1007/s00339-006-3503-6

Kocks, U.F., Tome, C.N., Wenk, H.-R., 1998. Texture and Anisotropy: Preferred Orientations in Polycrystals and their Effect on Materials Properties. Cambridge University Press, Cambridge, UK.

Krause, M.O., 1979. Atomic radiative and radiationless yields for K and L shells. J. Phys. Chem. Ref. Data 8, 307-327. doi:10.1063/1.555594

Larson, A.C., Von Dreele, R.B., Larson Von Dreele Allen.C., R.B., Larson Von Dreele, R.B., A.C., 2004. General Structure Analysis System (GSAS). Los Alamos, NM.

Levine, D., Steinhardt, P.J., 1984. Quasicrystals: A New Class of Ordered Structures. Phys. Rev. Lett. 53, 2477-2480. doi:10.1103/PhysRevLett.53.2477

Li, S., Van Houtte, P., Kalidindi, S.R., 2004. A quantitative evaluation of the deformation texture predictions for aluminium alloys from crystal plasticity finite element method. Model. Simul. Mater. Sci. Eng. 12, 845-870. doi:10.1088/0965-0393/12/5/006

Liss, K.D., Yan, K., 2010. Thermo-mechanical processing in a synchrotron beam. Mater. Sci. Eng. A 528, 11-27. doi:10.1016/j.msea.2010.06.017

Lloyd, L.T., Mueller, M.H., 1962. Recrystallization in rolled uranium sheet, ANL-63Z7. Argonne, IL.

Lokhov, A., 2010. The Supply of Medical Radioisotope: Review of Potential Molybdenum99/Technetium-99m Production Technologies. Nuclear Energy Agency.

Lonardelli, I., Wenk, H.R., Lutterotti, L., Goodwin, M., 2005. Texture analysis from synchrotron diffraction images with the Rietveld method: Dinosaur tendon and salmon scale. J. Synchrotron Radiat. 12, 354-360. doi:10.1107/S090904950500138X

Los Alamos National Laboratory, 2015. High Pressure Preferred Orientation at LANSCE [WWW Document]. URL http://lansce.lanl.gov/lujan/instruments/HIPPO.shtml

Losko, A.S., Vogel, S.C., Reiche, H.M., Nakotte, H., 2014. A six-axis robotic sample changer for high-throughput neutron powder diffraction and texture measurements. J. Appl. Crystallogr. 47, 2109-2112. doi:10.1107/S1600576714021797

Lutterotti, L., 2010a. Total pattern fitting for the combined size-strain-stress-texture determination in thin film diffraction. Nucl. Instruments Methods Phys. Res. Sect. B Beam Interact. with Mater. Atoms 268, 334-340. doi:10.1016/j.nimb.2009.09.053

Lutterotti, L., 2010b. Introduction to the Rietveld Method. Trento, Italy.

Lutterotti, L., Bortolotti, M., Ischia, G., Lonardelli, I., Wenk, H.R., 2007. Rietveld texture analysis from diffraction images. Zeitschrift fur Krist. Suppl. 1, 125-130. doi:10.1524/zksu.2007.2007.suppl_26.125

Lutterotti, L., Chateigner, D., Ferrari, S., Ricote, J., 2004. Texture, residual stress and structural analysis of thin films using a combined X-ray analysis. Thin Solid Films 450, 34-41. 
doi:10.1016/j.tsf.2003.10.150

Lutterotti, L., Matthies, S., Wenk, H.-R., Schultz, A.S., Richardson, J.. W., 1997. Combined texture and structure analysis of deformed limestone from time-of-flight neutron diffraction spectra. J. Appl. Phys. 81, 594-600. doi:10.1063/1.364220

Ma, D., Stoica, A.D., Wang, Z., Beese, A.M., 2017. Crystallographic texture in an additively manufactured nickel-base superalloy. Mater. Sci. Eng. A 684, 47-53. doi:10.1016/j.msea.2016.12.028

Mainprice, D., Bachmann, F., Hielscher, R., Schaeben, H., 2014. Descriptive tools for the analysis of texture projects with large data sets using MTEX: strength, symmetry and components. Geol. Soc. Spec. Publ. 409, 251-271.

Makarewicz, P.F., Solbrekken, G.L., 2013. Thermal/Mechanical/Hydraulic Experimental Tools for Mo-99 Production Target Analysis. University of Missouri.

Man, C.S., Huang, M., 2011. A simple explicit formula for the Voigt-Reuss-Hill average of elastic polycrystals with arbitrary crystal and texture symmetries. J. Elast. 105, 29-48. doi:10.1007/s10659-011-9312-y

Matthies, S., 2002. 20 Years WIMV, History, Experience and Contemporary Developments. Mater. Sci. Forum 408, 95-100. doi:10.4028/www.scientific.net/MSF.408-412.95

Matthies, S., Pehl, J., Wenk, H.R., Lutterotti, L., Vogel, S.C., 2005. Quantitative texture analysis with the HIPPO neutron TOF diffractometer. J. Appl. Crystallogr. 38, 462-475. doi:10.1107/S0021889805006187

Matthies, S., Wenk, H.R., 1992. Optimization of Texture Measurements by Pole Figure Coverage with Hexagonal Grids. Phys. Status Solidi A, 253-257.

McCabe, R.J., Capolungo, L., Marshall, P.E., Cady, C.M., Tomé, C.N., 2010. Deformation of wrought uranium: Experiments and modeling. Acta Mater. 58, 5447-5459. doi:10.1016/j.actamat.2010.06.021

McCabe, R.J., Richards, A.W., Coughlin, D.R., Clarke, K.D., Beyerlein, I.J., Knezevic, M., 2015. Microstructure effects on the recrystallization of low-symmetry alpha-uranium. J. Nucl. Mater. 465, 189-195. doi:10.1016/j.jnucmat.2015.04.055

McDonald, S.A., Reischig, P., Holzner, C., Lauridsen, E.M., Withers, P.J., Merkle, A.P., Feser, M., 2015. Non-destructive mapping of grain orientations in 3D by laboratory X-ray microscopy. Sci. Rep. 5, 14665. doi:10.1038/srep14665

Neuefeind, J., Feygenson, M., Carruth, J., Hoffmann, R., Chipley, K.K., 2012. The Nanoscale Ordered MAterials Diffractometer NOMAD at the Spallation Neutron Source SNS. Nucl. Instruments Methods Phys. Res. Sect. B Beam Interact. with Mater. Atoms 287, 68-75. doi:10.1016/j.nimb.2012.05.037

Onuki, Y., Hoshikawa, A., Sato, S., Xu, P., Ishigaki, T., Saito, Y., Todoroki, H., Hayashi, M., 2016. Rapid measurement scheme for texture in cubic metallic materials using time-of-flight neutron diffraction at iMATERIA. J. Appl. Cryst 49, 1579-1584. doi:10.1107/S160057671601164X

Pavithra, C.L.P., Sarada, B. V., Rajulapati, K. V., Ramakrishna, M., Gundakaram, R.C., Rao, T.N., Sundararajan, G., 2015. Controllable Crystallographic Texture in Copper Foils Exhibiting Enhanced Mechanical and Electrical Properties by Pulse Reverse Electrodeposition. Cryst. Growth Des. 15, 4448-4458. doi:10.1021/acs.cgd.5b00748

Perkin Elmer, 2011. XRD Detector Series.

Raghavan, N., Dehoff, R., Pannala, S., Simunovic, S., Kirka, M., Turner, J., Carlson, N., Babu, S.S., 2016. Numerical modeling of heat-transfer and the influence of process parameters on tailoring the grain morphology of IN718 in electron beam additive manufacturing. Acta Mater. 112, 303 314. doi:10.1016/j.actamat.2016.03.063 
Rechtien, J.J., Nelson, R.D., 1973. Phase transformations in uranium, plutonium, and neptunium. Metall. Trans. 4, 2755-2765.

Reiche, H.M., Carpenter, J.S., Stein, F., Tomida, T., Vogel, S.C., 2015. Metallurgical studies with the HIPPO diffractometer at LANSCE. Can. Metall. Q. 54, $2-8$. doi:10.1179/1879139514Y.0000000157

Rietveld, H.M., 1969. A profile refinement method for nuclear and magnetic structures. J. Appl. Crystallogr. 2, 65-71. doi:10.1107/S0021889869006558

Rietveld, H.M., 1967. Line profiles of neutron powder-diffraction peaks for structure refinement. Acta Crystallogr. 22, 151-152. doi:10.1107/S0365110X67000234

Rizzie, A.C., Watkins, T.R., Payzant, E.A., 2008. Elaboration of the Hexagonal Grid and Spiral Trace Schemes for Pole Figure Data Collection, International Centre for Diffraction Data. Denver, CO.

Roe, R.-J., 1965. Description of crystallite orientation in polycrystalline materials III, general solution to pole figure inversion. J. Appl. Phys. 36, 2024-2031.

Salice, R., Zein, F., 1961. Macro and microstructural changes of alpha-uranium upon thermal cycling, CNEN-52.

Saloman, E., Hubbell, J., 1988. X-ray attenuation cross sections for energies $100 \mathrm{eV}$ to $100 \mathrm{keV}$ and elements $Z=1$ to $Z=92$. At. Data Nucl. Data Tables 38, 1-196.

Schaeben, H., 1997. A Simple Standard Orientation Density Function:The Hyperspherical de la Vallee Poussin Kernel. Phys. Status Solidi B 200, 367-376.

Schell, N., Martins, R. V., Beckmann, F., Ruhnau, H.-U., Kiehn, R., Schreyer, A., 2008. The high energy materials science beamline at PETRA III. Mater. Sci. Forum 571-572, 261-266.

Schindelin, J., Rueden, C.T., Hiner, M.C., Eliceiri, K.W., 2015. The ImageJ ecosystem: An open platform for biomedical image analysis. Mol. Reprod. Dev. 82, 518-529. doi:10.1002/mrd.22489

Sears, V.F., 1992. Neutron scattering lengths and cross sections. Neutron News 3, 26-37. doi:10.1080/10448639208218770

Sherby, O., Bly, D., Wood, D., 1976. Plastic flow and strength of uranium and its alloys, in: Physical Metallurgy of Uranium Alloys. Brook Hill Publishing Co, Chestnut Hill, MA, pp. 311-348.

Simmons, G., Wang, H., 1971. Single Crystal Elastic Constants and Calculated Aggregate Properties: A Handbook. The Massachussetts Institute of Technology Press, Cambridge, MA.

Snelgrove, J.L., Hofman, G.L., Wiencek, T.C., Wu, C.T., Vandegrift, G.F., Aase, S., Buchholz, B.A., Dong, D.J., Leonard, R.A., Srinivasan, B., Wu, D., Suripto, A., Aliluddin, Z., 1995.

Development and Processing of LEU Targets for Mo-99 Production - Overview of the ANL Program. Argonne, IL.

Ståhl, K., 2008. Powder diffraction and the Rietveld Method. Lyngby, Denmark.

Steiner, M.A., McCabe, R.J., Garlea, E., Agnew, S.R., 2017. Monte Carlo Modeling of Recrystallization Processes in $\alpha$-Uranium. J. Nucl. Mater.

Stoica, G.M., Stoica, A.D., An, K., Ma, D., Vogel, S.C., Carpenter, J.S., Wang, X.L., 2014. Extracting grain-orientation-dependent data from in situ time-of-flight neutron diffraction. I. Inverse pole figures. J. Appl. Crystallogr. 47, 2019-2029. doi:10.1107/S1600576714023036

Sturcken, E.F., Walter, C.M., 1974. Texture and irradiation growth in EBR-II driver fuel. J. Nucl. Mater. 50, 69-82. doi:10.1016/0022-3115(74)90062-2

Tahreen, N., Zhang, D.F., Pan, F.S., Jiang, X.Q., Li, D.Y., Chen, D.L., 2016. Texture evolution and deformation activity of an extruded magnesium alloy: Effect of yttrium and deformation temperature. J. Alloys Compd. 688, 270-284. doi:10.1016/j.jallcom.2016.07.150 
Tarkowski, L., Laskosz, L., Bonarski, J.T., 2004. Optimization of X-Ray Pole Figure Measurement 444, 137-140. doi:10.4028/www.scientific.net/MSF.443-444.137

Thickness of Aluminum Foil [WWW Document], 2013. . U.S. Packag. Wrapping, LLC. URL http://www.uspackagingandwrapping.com/blog/Thickness-of-Aluminum-Foil.html

Turner, K., Solbrekken, G., Allen, C., 2009. Thermal-Mechanical Analysis of Annular Target Design for High Volume Production of Mo-99 Using LEU, in: Proceedings of the ASME - International Mechanical Engineering Congress \& Exposition.

Ullemeyer, K., Spalthoff, P., Heinitz, J., Isakov, N.N., Nikitin, A.N., Weber, K., 1998. The SKat texture diffractometer at the pulsed reactor IBR-2 at Dubna: Experimental layout and first measurements. Nucl. Instruments Methods Phys. Res. Sect. A Accel. Spectrometers, Detect. Assoc. Equip. 412, 80-88. doi:10.1016/S0168-9002(98)00340-4

Usher, T.-M., 2016. Local and Average Structure in Ferroelectrics under Perturbing Fields. North Carolina State University.

Van Houtte, P., 1983. The Use of a Quadratic Form for the Determination of Non-Negative Texture Functions. Textures Microstruct. 6, 1-19.

Van Houtte, P., Li, S., Seefeldt, M., Delannay, L., 2005. Deformation texture prediction: From the Taylor model to the advanced Lamel model, International Journal of Plasticity. doi:10.1016/j.ijplas.2004.04.011

Van Swygenhoven, H., Van Petegem, S., 2013. In-situ mechanical testing during X-ray diffraction. Mater. Charact. 78, 47-59. doi:10.1016/j.matchar.2012.12.010

Vogel, S.C., Hartig, C., Lutterotti, L., Von Dreele, R.B., Wenk, H.-R., Williams, D.J., 2004. Texture measurements using the new neutron diffractometer HIPPO and their analysis using the Rietveld method. Powder Diffr. 19, 65. doi:10.1154/1.1649961

Wagermaier, W., Gupta, H.S., Gourrier, A., Paris, O., Roschger, P., Burghammer, M., Riekel, C., Fratzl, P., 2007. Scanning texture analysis of lamellar bone using microbeam synchrotron X-ray radiation. J. Appl. Crystallogr. 40, 115-120. doi:10.1107/S0021889806044888

Wenk, H.-R., Lutterotti, L., Vogel, S., 2003. Texture analysis with the new HIPPO TOF diffractometer. Nucl. Instruments Methods Phys. Res. Sect. A Accel. Spectrometers, Detect. Assoc. Equip. 515, 575-588. doi:10.1016/j.nima.2003.05.001

Wenk, H.-R., Lutterotti, L., Vogel, S.C., 2010. Rietveld texture analysis from TOF neutron diffraction data. Powder Diffr. 25, 283-296. doi:10.1154/1.3479004

Wenk, H.-R., Matthies, S., Lutterotti, L., 1994. Texture Analysis from Diffraction Spectra. Mater. Sci. Forum 157, 473-480. doi:10.4028/www.scientific.net/MSF.157-162.473

Wenk, H.R., Cont, L., Xie, Y., Lutterotti, L., Ratschbacher, L., Richardson, J., 2001. Rietveld texture analysis of Dabie Shan eclogite from TOF neutron diffraction spectra. J. Appl. Crystallogr. 34, 442-453. doi:10.1107/S0021889801005635

Whipple, C., Larson, S., 2009. Medical Isotope Production Without Highly Enriched Uranium. The National Academies Press.

Will, G., 2006. Powder Diffraction: The Rietveld Method and the Two-Stage Method, Powder Diffraction. doi: 10.1007/3-540-27986-5

Wrights, S.I., Zhao, J., Adams, B.L., 1991. Automated determination of lattice orientation from electron backscattered kikuchi diffraction patterns. Textures Microstruct. 13, 123-131.

Yamagishi, H., Toh, K., Nakamura, T., Sakasai, K., Soyama, K., 2011. Simulation program for multiwire-type two-dimensional neutron detector with individual readout. J. Instrum. 6, 1-7. doi:10.1088/1748-0221/6/12/C12025

Yan, K., Liss, K.-D., Timokhina, I.B., Pereloma, E. V., 2016. In situ synchrotron X-ray diffraction 
studies of the effect of microstructure on tensile behavior and retained austenite stability of thermo-mechanically processed transformation induced plasticity steel. Mater. Sci. Eng. A 662, 185-197. doi:10.1016/j.msea.2016.03.048

Yemel'yanov, V.S., Yevstyukhin, A.I., Dunworth, J.V., 1969. The Metallurgy of Nuclear Fuel: Properties and Principles of the Technology of Uranium, Thorium and Plutonium. Pergamon.

Zecevic, M., Knezevic, M., Beyerlein, I.J., McCabe, R.J., 2016a. Origin of texture development in orthorhombic uranium. Mater. Sci. Eng. A 665, 108-124. doi:10.1016/j.msea.2016.04.018

Zecevic, M., Knezevic, M., Beyerlein, I.J., McCabe, R.J., 2016b. Texture formation in orthorhombic alpha-uranium under simple compression and rolling to high strains. J. Nucl. Mater. 473, 130144.

Zener, C., 1948. Elasticity and Anelasticity of Metals. The University of Chicago Press, Chicago, IL.

Zhang, J., Liu, Y., Ren, Y., Huan, Y., Hao, S., Yu, C., Shao, Y., Ru, Y., Jiang, D., Cui, L., 2014. In situ synchrotron X-ray diffraction study of deformation behavior and load transfer in a Ti2NiNiTi composite. Appl. Phys. Lett. 105. doi:10.1063/1.4892352 